\title{
A GAME THEORETICAL APPROACH TO THE ALGEBRAIC COUNTERPART OF THE WAGNER HIERARCHY: PART II
}

\author{
JÉRÉmie CABessa ${ }^{1}$ and JaCQues Duparc ${ }^{1}$
}

\begin{abstract}
The algebraic counterpart of the Wagner hierarchy consists of a well-founded and decidable classification of finite pointed $\omega$-semigroups of width 2 and height $\omega^{\omega}$. This paper completes the description of this algebraic hierarchy. We first give a purely algebraic decidability procedure of this partial ordering by introducing a graph representation of finite pointed $\omega$-semigroups allowing to compute their precise Wagner degrees. The Wagner degree of any $\omega$-rational language can therefore be computed directly on its syntactic image. We then show how to build a finite pointed $\omega$-semigroup of any given Wagner degree. We finally describe the algebraic invariants characterizing every degree of this hierarchy.
\end{abstract}

Mathematics Subject Classification. O3D55, 20M35, 68Q70, $91 \mathrm{~A} 65$.

\section{INTRODUCTION}

In 1979, Wagner defined a reduction relation on $\omega$-rational languages by analyzing the graphs of their underlying Muller automata. The collection of $\omega$-rational languages ordered by this reduction is nowadays called the Wagner hierarchy, and was proven to be a well-founded and decidable partial ordering of height $\omega^{\omega}$ [21]. But the Wagner hierarchy also coincides with the restriction of the Wadge hierarchy [20] - the most refined hierarchy in descriptive set theory - to $\omega$-rational languages, and therefore refines considerably the very lower levels of the Borel hierarchy. The Wagner reduction thus corresponds to the Wadge or the continuous

\footnotetext{
Keywords and phrases. $\omega$-automata, $\omega$-rational languages, $\omega$-semigroups, infinite games, hierarchical games, Wadge game, Wadge hierarchy, Wagner hierarchy.

1 University of Lausanne, Faculty of Business and Economics, HEC - ISI, 1015 Lausanne, Switzerland; Jeremie.Cabessa@unil.ch
} 
reduction; but it also coincides with the sequential reduction - a reduction defined by means of automata - on the class of $\omega$-rational languages ([16], Thm. 5.2, p. 209).

The Wagner hierarchy has been thoroughly investigated since then. Wilke and Yoo described an efficient algorithm computing the Wagner degree of any $\omega$-rational language in polynomial time [23], and Selivanov proposed a purely descriptive set theoretical formulation of this hierarchy [18].

The present series of papers is concerned with the algebraic approach to $\omega$ rational languages. In this context, Pin introduced the structure of an $\omega$-semigroup [16] (extensions of semigroups equipped with an infinite product) as an algebraic counterpart of Büchi automata, and Wilke was the first to prove that $\omega$ rational languages are also exactly the ones recognized by finite $\omega$-semigroups [22]. These algebraic structures present some relevant properties: for instance, the existence of a minimal $\omega$-semigroup recognizing a given $\omega$-rational language - the syntactic image of this language; they also reveal interesting classification properties, for example an $\omega$-language is first-order definable if and only if it is recognized by an aperiodic $\omega$-semigroup $[13,15,19]$, a generalization to infinite words of Schützenberger and McNaughton's famous result. The problem of classifying finite $\omega$-semigroups in such a refined way as Wagner did for $\omega$-rational languages thence appeared naturally.

Carton and Perrin [2-4], and Duparc and Riss [8] studied an algebraic description of the Wagner hierarchy in connection with the theory of $\omega$-semigroup. But their results still fail to provide an algorithm that computes the Wagner degree of an $\omega$-rational language directly on a corresponding $\omega$-semigroup, and in particular on the syntactic $\omega$-semigroup of this language.

These two papers provide an algebraic description of the Wagner hierarchy. In the first paper of this series, we gave a construction of the algebraic counterpart of the Wagner hierarchy. We defined a reduction relation on finite $\omega$-semigroups by transposing Wadge games from the $\omega$-language to the $\omega$-semigroup context, and we proved that the collection of finite pointed $\omega$-semigroups ordered by this reduction was precisely isomorphic to the Wagner hierarchy - namely a decidable partial ordering of height $\omega^{\omega}$. The present paper completes this description. We first expose a decidability procedure based on a graph representation of finite pointed $\omega$-semigroups. This algorithm can therefore compute the Wagner degree of any $\omega$-rational language directly on its syntactic image, and consists of a reformulation in this algebraic context of Wagner's naming procedure [21]. We then show how to build a finite pointed $\omega$-semigroups of any given Wagner degree. We finally describe the algebraic invariant characterizing the Wagner degree of every finite $\omega$ semigroup. These invariants are also a reformulation in this context of the notions of maximal $\xi$-chains presented in [8], or maximal $\mu_{\alpha}$-alternating trees described in [18], or also maximal binary tree-like sequences of superchains described in [21]. 


\section{Preliminaries}

\subsection{ORdinals}

We refer to $[11,12,14]$ for a complete presentation of ordinals and ordinal arithmetic. We simply recall that, up to isomorphism, an ordinal is just a linearly ordered well-founded set. The first infinite ordinal, denoted by $\omega$, is the set of all integers, and the ordinal $\omega^{\omega}$ is defined as $\sup \left\{\omega^{n} \mid n<\omega\right\}$. Any ordinal $\xi$ strictly below $\omega^{\omega}$ can be uniquely written by its Cantor normal form of base $\omega$ as follows:

$$
\xi=\omega^{n_{k}} \cdot p_{k}+\cdots+\omega^{n_{0}} \cdot p_{0},
$$

for some unique strictly descending sequence of integers $n_{k}>\ldots>n_{0} \geq 0$ and some $p_{i}>0$, for all $i$. We finally recall that the ordinal sum satisfies the property $\omega^{p}+\omega^{q}=\omega^{q}$, whenever $q>p$.

This paper only involves ordinals strictly below $\omega^{\omega}$ and we choose to present an alternative characterization of those ones. The set of ordinals strictly below $\omega^{\omega}$ (that is $\omega^{\omega}$ itself) is isomorphic to the set

$$
\operatorname{Ord}_{<\omega^{\omega}}=\{0\} \cup \bigcup_{k \in \mathbb{N}}\left(\mathbb{N} \backslash\{0\} \times \mathbb{N}^{k}\right)
$$

- that is the set containing the integer 0 plus all finite nonempty sequences of integers whose left most component is strictly positive - equipped with the following ordering: 0 is the least element and given any two sequences $\alpha=\left(a_{0}, \ldots, a_{m}\right), \beta=$ $\left(b_{0}, \ldots, b_{n}\right) \in \operatorname{Ord}_{<\omega^{\omega}}$, then

$$
\alpha<\beta \text { if and only if }\left\{\begin{array}{l}
\text { either } m<n, \\
\text { or } m=n \text { and } \alpha<_{\text {lex }} \beta
\end{array}\right.
$$

where $<_{\text {lex }}$ denote the lexicographic order. This relation is clearly a well-ordering. For instance, one has $(7,3,0,0,1)<(1,0,0,0,0,0)$ and $(7,3,0,0,1)<(7,3,1,0,1)$. As usual, given such a sequence $\alpha$, the $i$ th element of $\alpha$ is denoted by $\alpha(i)$. For example, if $\alpha=(3,0,0,2,1)$, then $\alpha(0)=3$ and $\alpha(3)=2$.

Every ordinal $\xi<\omega^{\omega}$ can then be associated in a unique way with an element of $\operatorname{Ord}_{<\omega^{\omega}}$ as described hereafter: the ordinal 0 is associated with 0 , and every ordinal $0<\xi<\omega^{\omega}$ with Cantor normal form $\omega^{n_{k}} \cdot p_{k}+\cdots+\omega^{n_{0}} \cdot p_{0}$ is associated with the sequence of integers $\bar{\xi}$ of length $n_{k}+1$ defined by $\bar{\xi}\left(n_{k}-i\right)$ being the multiplicative coefficient of the term $\omega^{i}$ in this Cantor normal form. The sequence $\bar{\xi}$ is thence an encoding of the Cantor normal form of $\xi$. For instance, the ordinal $\omega^{4} \cdot 3+\omega^{3} \cdot 5+\omega^{0} \cdot 1$ corresponds to the sequence $(3,5,0,0,1)$. The ordinal $\omega^{n}$ corresponds the sequence $(1,0,0, \ldots, 0)$ containing $n$ 's. This correspondence is an isomorphism from $\omega^{\omega}$ into $\operatorname{Ord}_{<\omega^{\omega}}$, and from this point onward, we will make no more distinction between non-zero ordinals strictly below $\omega^{\omega}$ and their corresponding sequences of integers. 
In this framework, the ordinal sum on sequences of integers is defined as follows: given $\alpha=\left(a_{0}, \ldots, a_{m}\right), \beta=\left(b_{0}, \ldots, b_{n}\right) \in \operatorname{Ord}_{<\omega^{\omega}}$, then

$$
\alpha+\beta= \begin{cases}\beta & \text { if } m<n, \\ (\alpha(0), \ldots, \alpha(n-m-1), \alpha(n-m)+\beta(0), \beta(1), \ldots, \beta(n)) & \text { if } m \geq n .\end{cases}
$$

For instance, one has

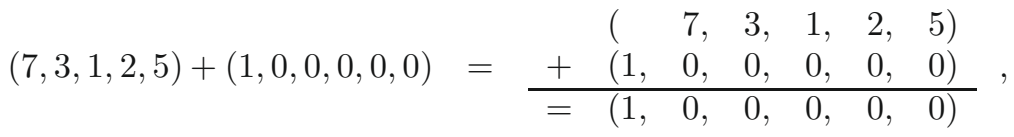

$$
\begin{aligned}
& (7,3,1, \quad 2,5)
\end{aligned}
$$

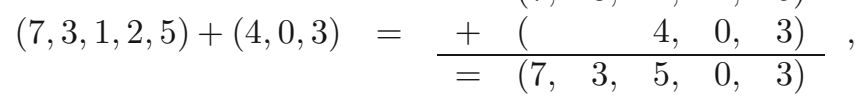

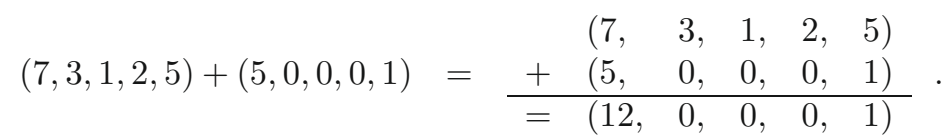

As usual, the multiplication by an integer is defined by induction via the ordinal sum.

A signed ordinal is a pair $(\varepsilon, \xi)$, where $\xi$ is an ordinal strictly below $\omega^{\omega}$ and $\varepsilon \in\{+,-, \pm\}$. It will be denoted by $[\varepsilon] \xi$ instead. Signed ordinal are equipped with the following partial ordering: $[\varepsilon] \xi<\left[\varepsilon^{\prime}\right] \xi^{\prime}$ if and only if $\xi<\xi^{\prime}$. Therefore the signed ordinals $[+] \xi,[-] \xi$, and $[ \pm] \xi$ are all three incomparable.

Given an ordinal $0<\xi<\omega^{\omega}$ with Cantor normal form $\omega^{n_{k}} \cdot p_{k}+\cdots+\omega^{n_{0}} \cdot p_{0}$, the playground of $\xi$, denoted by $p g(\xi)$, is simply defined as the integer $n_{0}$. When regarded as a sequence of integers, the playground of $\xi$ is the number of successive 0 's from the right end of $\xi$. For instance, $p g((2,4,0,5,0,0))=2$. Finally, given a signed ordinal $[\varepsilon] \xi$ with $\varepsilon \in\{+,-\}$ and Cantor normal form $\xi=\omega^{n_{k}} \cdot p_{k}+$ $\cdots+\omega^{n_{0}} \cdot p_{0}$, a cut of $[\varepsilon] \xi$ is a signed ordinal $\left[\varepsilon^{\prime}\right] \xi^{\prime}<[\varepsilon] \xi$ satisfying the following properties:

(1) $\xi^{\prime}=\omega^{n_{k}} \cdot p_{k}+\cdots+\omega^{n_{i}} \cdot q_{i}$, for some $0 \leq i \leq k$ and $q_{i} \leq p_{i}$;

(2) if $n_{i}=n_{0}$, then $\varepsilon^{\prime}=\varepsilon$ if and only if $p_{i}$ and $q_{i}$ have the same parity; whereas if $n_{i}>n_{0}$, then $\varepsilon^{\prime} \in\{+,-\}$ with no restriction.

If $\xi$ is regarded as the sequence of integers $\left(a_{0}, \ldots, a_{n}\right)$, a cut of $[\varepsilon] \xi$ is a signed ordinal $\left[\varepsilon^{\prime}\right]\left(b_{0}, \ldots, b_{n}\right)<[\varepsilon]\left(a_{0}, \ldots, a_{n}\right)$ satisfying the following properties:

(1) there exists an index $i$ such that: firstly, $b_{j}=a_{j}$, for each $0 \leq j<i$; secondly, $b_{i}<a_{i}$; thirdly, $b_{j}=0$, for each $i<j \leq n$;

(2) if $p g\left(a_{0}, \ldots, a_{n}\right)=p g\left(b_{0}, \ldots, b_{n}\right)=p$, then $\varepsilon^{\prime}=\varepsilon$ if and only if $a_{n-p}$ and $b_{n-p}$ have the same parity; whereas if $p g\left(a_{0}, \ldots, a_{n}\right) \neq p g\left(b_{0}, \ldots, b_{n}\right)$, then $\varepsilon^{\prime} \in\{+,-\}$ with no restriction.

For instance, the successive cuts of the signed ordinal $[+](2,0,3,0)$ are $[-](2,0,2,0)$, $[+](2,0,1,0),[+](2,0,0,0),[-](2,0,0,0),[+](1,0,0,0)$, and $[-](1,0,0,0)$. As another example, the cuts of the signed ordinal $[-](4,2,0,3,0)$ are all listed below 
by decreasing order (i.e. $[\varepsilon] \xi$ can access $\left[\varepsilon^{\prime}\right] \xi^{\prime}$ iff $[\varepsilon] \xi>\left[\varepsilon^{\prime}\right] \xi^{\prime}$ ).

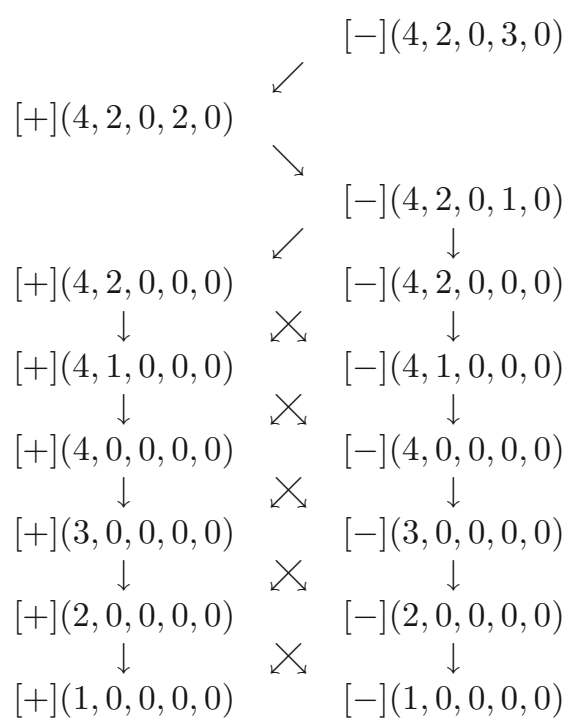

\subsection{Semigroups}

We refer to [17] for all basic definitions concerning semigroups, Green preorders $\leq_{\mathcal{L}}, \leq_{\mathcal{R}}, \leq_{\mathcal{H}}$, as well as their corresponding equivalence relations $\mathcal{L}, \mathcal{R}, \mathcal{H}$. Given a semigroup $S$, the set of idempotents of $S$ is denoted by $E(S)$, or simply by $E$ when the semigroup involved is clear from the context. The restriction of the preorder $\leq_{\mathcal{H}}$ to the set $E(S)$ is a partial order, called the natural order on $E(S)[16,17]$, and denoted by $\leq$. If $S$ is a finite semigroup, there exists an integer $\pi$ such that, for each $s \in S$, the element $s^{\pi}$ is idempotent [17]. The least integer satisfying this property is called the exponent of $S$.

A pair $(s, e) \in S^{2}$ is called a linked pair if $s e=s$ and $e$ is idempotent. The elements $s$ and $e$ are respectively called the prefix and the idempotent of the linked pair. The set of all prefixes of linked pairs of $S$ is denoted by $P(S)$, or simply by $P$ if the semigroup involved is clear from the context. The set of idempotents associated with a given prefix $s$ is defined by $E(s, S)=\{e \in E(S) \mid s e=s\}$, and is also simply denoted by $E(s)$ when there is no ambiguity. Moreover, two linked pairs $(s, e)$ and $\left(s^{\prime}, e^{\prime}\right)$ of $S^{2}$ are said to be conjugate, denoted by $(s, e)={ }_{c}\left(s^{\prime}, e^{\prime}\right)$, if there exist $x, y \in S$ such that $e=x y, e^{\prime}=y x$, and $s^{\prime}=s x$. The conjugacy relation between linked pairs is an equivalence relation [16], and the conjugacy class of a linked pair $(s, e)$ will be denoted by $[s, e]$.

In [16], Chapter II - 2 fully describes the specific properties of infinite words over finite semigroups. We recall some of these useful results. If $\alpha=\left(x_{n}\right)_{n \in \mathbb{N}}$ and $\beta=\left(y_{n}\right)_{n \in \mathbb{N}}$ are two infinite words of a semigroup $S$, then $\beta$ is said to be a factorization of $\alpha$ if there exists a strictly increasing sequence of integers $\left(k_{n}\right)_{n \geq 0}$ 
such that $y_{0}=x_{0} \cdots x_{n_{0}-1}$ and $y_{n+1}=x_{k_{n}} \cdots x_{k_{n+1}-1}$, for each $n \geq 0$. The next proposition tightly binds infinite words over finite semigroups to linked pairs.

Proposition 1.1 (see [16], pp. 78-79). Let $S$ be a finite semigroup, and $\left(s_{n}\right)_{n>0}$ be an infinite sequence of elements of $S$. Then there exist a linked pair $(s, e) \in \bar{S}^{2}$ and a strictly increasing sequence of integers $\left(k_{n}\right)_{n \geq 0}$, such that $s_{0} s_{1} \ldots s_{k_{0}-1}=s$ and $s_{k_{n}} s_{k_{n}+1} \ldots s_{k_{n+1}-1}=e$, for all $n \geq 0$.

In this case, the infinite word $\left(s_{n}\right)_{n \geq 0}$ is said to be associated with the linked pair $(s, e)$. In a finite semigroup $S$, there exists an infinite word which can be associated with different linked pairs if and only if these linked pairs are conjugate [16]. This property ensures the existence of a surjective mapping from the set of infinite words onto the set of classes of linked pairs of $S$, which maps every infinite words to its associated conjugacy class.

\section{3. $\omega$-Semigroups}

We refer to [16] for basic definitions and results concerning $\omega$-semigroups. We recall that $\omega$-rational languages are exactly those recognized by finite $\omega$ semigroups [16,22]. Hence in this paper, we particularly focus on finite $\omega$-semigroups, and it is proven in [16] that every finite $\omega$-semigroup $S$ is entirely and uniquely determined by the infinite products of the form $\pi_{S}(s, s, s, \ldots)$, denoted by $s^{\omega}$. More precisely, given a finite $\omega$-semigroup $S=\left(S_{+}, S_{\omega}\right)$, and an infinite sequence $\left(s_{i}\right)_{i \in \mathbb{N}}$ of elements of $S_{+}$, one has $\pi_{S}\left(s_{0}, s_{1}, s_{2}, \ldots\right)=s e^{\omega}$, for any linked pair $(s, e)$ associated with $\left(s_{i}\right)_{i \in \mathbb{N}}$ in the sense of Proposition 1.1 - the value of this infinite product is indeed independent of the associated linked pair chosen [16]. We then have the following consequence:

Lemma 1.2. Let $S=\left(S_{+}, S_{\omega}\right)$ be a finite $\omega$-semigroup, and let $\alpha$ and $\beta$ be infinite words of $S_{+}^{\omega}$ such that $\beta$ is a factorization of $\alpha$. Then $\pi_{S}(\alpha)=\pi_{S}(\beta)$.

Proof. Let $(s, e)$ be a linked pair of $S_{+}^{2}$ associated with $\beta$. Therefore $\pi_{S}(\beta)=s e^{\omega}$. Since $\beta$ is a factorization of $\alpha$, then $(s, e)$ is also associated with $\alpha$. Therefore $\pi_{S}(\alpha)=s e^{\omega}=\pi_{S}(\beta)$.

In addition, we recall that the definition of a pointed $\omega$-semigroup can be straightforwardly adapted from the definition of a pointed semigroup: a pointed $\omega$-semigroup is a pair $(S, X)$, where $S$ is an $\omega$-semigroup and $X$ is a subset of $S$. The definitions of $\omega$-subsemigroups, quotient, and division can then be easily reformulated in this pointed context. Given a pointed $\omega$-semigroup $(S, X)$, with $S=\left(S_{+}, S_{\omega}\right)$ and $X \subseteq S_{\omega}$, and given an element $u$ of $S_{+}$, we set $u X=\{u \alpha \in$ $\left.S_{\omega} \mid \alpha \in X\right\}$, and $u^{-1} X=\left\{\alpha \in S_{\omega} \mid u \alpha \in X\right\}$.

Finally, a pointed $\omega$-semigroup $(S, X)$ will be called Borel if the preimage $\pi_{S}^{-1}(X)$ is a Borel subset of $S_{+}^{\omega}$, where $S_{+}^{\omega}$ is equipped with the product topology of the discrete topology on $S_{+}$. Notice that every finite pointed $\omega$-semigroup is Borel, since its preimage by the infinite product is $\omega$-rational, hence Borel (more precisely Boolean combination of $\boldsymbol{\Sigma}_{\mathbf{2}}^{\mathbf{0}}$ ) [16]. 


\section{The $\mathbb{S} G$-HIERARCHY}

Let $(S, X)$ and $(T, Y)$ be two pointed $\omega$-semigroups, where $S=\left(S_{+}, S_{\omega}\right)$ and $X \subseteq S_{\omega}$, and $T=\left(T_{+}, T_{\omega}\right)$ and $Y \subseteq T_{\omega}$. The game $\mathbb{S} \mathbb{G}((S, X),(T, Y))[1]$ is an infinite two-player game with perfect information, where Player $I$ is in charge of $X$, Player II is in charge of $Y$, and players I and II alternately play elements of $S_{+}$and $T_{+} \cup\{-\}$, respectively. Player I begins. Unlike Player I, Player II is allowed to skip her turn by playing the symbol "-", provided she plays infinitely many moves. After $\omega$ turns each, players I and II produced two infinite sequences $\left(s_{0}, s_{1}, \ldots\right) \in S_{+}^{\omega}$ and $\left(t_{0}, t_{1}, \ldots\right) \in T_{+}^{\omega}$, respectively. The winning condition is given as follows: Player II wins $\mathbb{S} G((S, X),(T, Y))$ if and only if $\pi_{S}\left(s_{0}, s_{1}, \ldots\right) \in$ $X \Leftrightarrow \pi_{T}\left(t_{0}, t_{1}, \ldots\right) \in Y$. From this point forward, the game $\mathbb{S} \mathbb{G}((S, X),(T, Y))$ will be denoted by $\mathbb{S} G(X, Y)$ and the $\omega$-semigroups involved will always be known by the context. A play of this game is illustrated below.

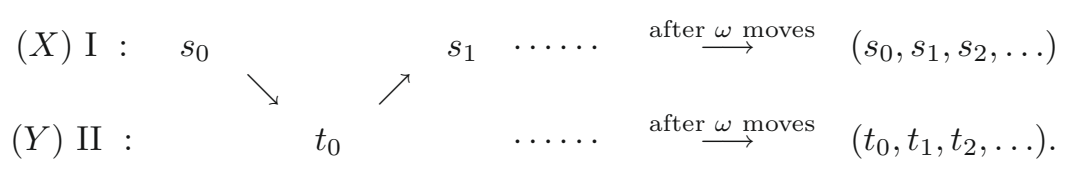

A player is said to be in position $s$ if the product of his/her previous moves $\left(s_{1}, \ldots, s_{n}\right)$ is equal to $s$. A strategy for Player $\mathrm{I}$ is a mapping $\sigma:\left(T_{+} \cup\{-\}\right)^{*} \longrightarrow$ $S_{+}$. A strategy for Player II is a mapping $\sigma: S_{+}^{+} \longrightarrow T_{+} \cup\{-\}$. A winning strategy for a given player is a strategy such that this player always wins when using it. Notice finally that a player in charge of the set $s^{-1} X$ is exactly as strong as a player in charge of $X$ but having already reached the position $s$.

The $\mathbb{S} G$-reduction over pointed $\omega$-semigroups is defined via this infinite game as follows: we say that $(S, X)$ is $\mathbb{S} G$-reducible to $(T, Y)$, simply denoted by $X \leq_{S G} Y$, if and only if Player II has a winning strategy in $\mathbb{S} G(X, Y)$. As usual, we then set $X \equiv_{S G} Y$ if and only if both $X \leq_{S G} Y$ and $Y \leq_{S G} X$, and $X<_{S G} Y$ if and only if both $X \leq_{S G} Y$ and $X \not_{S G} Y$. An $\omega$-subset $X$ is called self-dual if $X \leq_{S G} X^{c}$ and non-self-dual otherwise. The relation $\leq_{S G}$ is reflexive and transitive, hence $\equiv_{S G}$ is an equivalence relation.

The collection of Borel pointed $\omega$-semigroups ${ }^{1}$ ordered by the $\leq_{S G}$-relation is called the $\mathbb{S} G$-hierarchy, in order to underline the semigroup approach. Notice that the restriction of the $\mathbb{S} \mathbb{G}$-hierarchy to Borel pointed free $\omega$-semigroups is exactly the Borel Wadge hierarchy. When restricted to finite pointed $\omega$-semigroups, this hierarchy will be called the $\mathbb{F} \mathbb{S} G$-hierarchy, in order to underline the finiteness of the $\omega$-semigroups involved ${ }^{2}$. The $\mathbb{S} \mathbb{G}$-games over Borel $\omega$-subsets are determined, and as a corollary, one can prove that, up to complementation and $\mathbb{S} \mathbb{G}$-equivalence, the $\mathbb{S} G$-hierarchy is a well-ordering. Therefore, there exist a unique ordinal, called the height of the $\mathbb{S} G$-hierarchy, and a mapping $d_{S G}$ from the $\mathbb{S} \mathbb{G}$-hierarchy onto its

\footnotetext{
$1_{i . e .}$ pointed $\omega$-semigroups with Borel $\omega$-subsets.

2 Since every finite pointed $\omega$-semigroup is Borel, the $\mathbb{F} \mathbb{S} G$-hierarchy contains all finite pointed $\omega$-semigroups.
} 


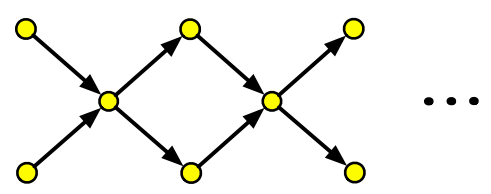

FIGURE 1. The $\mathbb{S} G$-hierarchy.

height, called the $\mathbb{S} G$-degree, such that $d_{S G}(X)<d_{S G}(Y)$ if and only if $X<_{S G} Y$, and $d_{S G}(X)=d_{S G}(Y)$ if and only if either $X \equiv_{S G} Y$ or $X \equiv_{S G} Y^{c}$, for every Borel $\omega$-subsets $X$ and $Y$. The wellfoundedness of the $\mathbb{S} G$-hierarchy ensures that the $\mathbb{S} G$-degree can be defined by induction as follows:

$$
d_{S G}(X)= \begin{cases}0 & \text { if } X=\emptyset \text { or } X=\emptyset^{c} \\ \sup \left\{d_{S G}(B)+1: B<_{S G} A\right\} & \text { otherwise }\end{cases}
$$

The $\mathbb{S} G$-hierarchy was proven to have the same familiar "scaling shape" as the Borel hierarchy or the Wadge hierarchy: an increasing sequence of non-self-dual sets with self-dual sets in between, as illustrated in Figure 1, where circles represent the $\equiv_{S G}$-equivalence classes of Borel $\omega$-subsets, and arrows stand for the $<_{S G^{-}}$ relation.

The $\omega$-subsets involved in finite pointed $\omega$-semigroups are necessarily Borel, so that the $\mathbb{F} \mathbb{S} G$-hierarchy is actually a restriction of the $\mathbb{S} G$-hierarchy. More precisely, in the first paper of this series, the $\mathbb{F} \mathbb{S G}$-hierarchy was proven to be the exact algebraic counterpart of the Wagner hierarchy in the following sense:

Theorem 2.1. The Wagner hierarchy and the $\mathbb{F} \mathbb{S G}$-hierarchy are isomorphic.

The isomorphism was indeed given by the mapping associating every $\omega$-rational language with its syntactic pointed image. As direct consequences, the $\mathbb{F} \mathbb{S} G$ hierarchy has height $\omega^{\omega}$, and it is decidable. This paper provides a detailed description as well as a decidability procedure of this hierarchy.

In this context, the following results present a useful game theoretical characterization of the self-dual and non-self-dual $\omega$-subsets. We first need to introduce the following notions. Given a finite $\omega$-semigroup $S=\left(S_{+}, S_{\omega}\right)$, an $\omega$-subset $X \subseteq S_{\omega}$, and two elements $s, e \in S_{+}$: we say that $s$ is a prefix position if $s$ is a prefix of some linked pair of $S_{+}^{2}$; we say that $e$ is a waiting move for the prefix position $s$ if $(s, e)$ is a linked pair; we say that $s$ is a critical position for $X$ if $s^{-1} X<_{S G} X$. We finally define the imposed game $\overline{\mathbb{S G}}\left({ }_{-},-\right.$), very similar to $\mathbb{S G}\left({ }_{-},-\right)$, except that Player I is allowed to skip his turn, provided he plays infinitely often, whereas Player II is not allowed to do so, and is forced to play from one prefix position to another. This infinite game induces the reduction relation $\leq \overline{S G}$ defined as usual by $X \leq \overline{S G} Y$ if and only if Player II has a winning strategy in $\overline{\mathbb{S} G}(X, Y)$.

The following results prove that an $\mathbb{S} G$-player is in charge of a self-dual $\omega$ subset if and only if $\mathrm{s} /$ he his forced to reach some critical position for this set. 
Equivalently, an $\mathbb{S G}$-player is in charge of a non-self-dual $\omega$-subset if and only if $\mathrm{s} /$ he has the possibility to indefinitely remain as strong as in her/his initial position. As a corollary, we show that every self-dual set can be written as a finite union of $<_{S G}$-smaller non-self-duals sets.

Lemma 2.2. Let $S=\left(S_{+}, S_{\omega}\right)$ and $T=\left(T_{+}, T_{\omega}\right)$ be two finite $\omega$-semigroups, let $X \subseteq S_{\omega}$ and $Y \subseteq T_{\omega}$, and let $s$ be a prefix of a linked pair of $T_{+}^{2}$. Then

$$
X \leq_{S G} s^{-1} Y \text { if and only if } X \leq \overline{S G} s^{-1} Y \text {. }
$$

Proof.

$(\Leftarrow)$ Notice that Player II is more constrained in the $\overline{\mathbb{S G}}$-game than in the $\mathbb{S G}$ game. Hence, if Player II has a winning strategy in $\overline{\mathbb{S G}}\left(X, s^{-1} Y\right)$, then she also has a winning strategy in $\mathbb{S} G\left(X, s^{-1} Y\right)$.

$\Leftrightarrow)$ In the game $\overline{\mathbb{S G}}\left(X, s^{-1} Y\right)$, we may assume that Player II is in charge of the subset $Y$, and is already in the prefix position $s$ in the beginning of the play. Now, given a winning strategy $\sigma$ for Player II in $\mathbb{S G}\left(X, s^{-1} Y\right)$, we describe a winning strategy for Player II in $\overline{\mathbb{S G}}\left(X, s^{-1} Y\right)$. For that purpose, let $a_{0}, a_{1}, a_{2}, \ldots$ denote the subsequence of non-skipping moves played by Player I in $\overline{\mathbb{S G}}\left(X, s^{-1} Y\right)$, and let $b_{i}=\sigma\left(a_{0}, \ldots, a_{i}\right)$ be the answers of Player II in the other game $\mathbb{S} G\left(X, s^{-1} Y\right)$, for all $i \geq 0$. Then, while I begins to play his very first successive moves, II first waits in her initial prefix position $s$ by playing an idempotent $e$ such that $s e=s$. As soon as I's moves induce an answer $b_{0} \cdots b_{m}$ such that $b_{0} \cdots b_{k-1}=s^{\prime}$, $b_{k} \cdots b_{m}=e^{\prime}$, and $\left(s^{\prime}, e^{\prime}\right)$ is a linked pair, then II either stays in (if $\left.s^{\prime}=s\right)$ or reaches position $s^{\prime}$. She then waits in this position by playing the idempotent $e^{\prime}$ until I's moves induce another finite word $b_{0} \cdots b_{n}$, with $n>m$, such that $b_{0} \cdots b_{m+i}=s^{\prime \prime}, b_{m+i+1} \cdots b_{n}=e^{\prime \prime}, i \geq 0$, and $\left(s^{\prime \prime}, e^{\prime \prime}\right)$ is a linked pair. As before, she either stays in or reaches position $s^{\prime \prime}$ by playing the element $\left(b_{m+1} \cdots b_{m+i}\right)$, when it exists, and waits in this position for another similar situation by playing the idempotent $e^{\prime \prime}$. And so on and so forth. Proposition 1.1 shows that this configuration is forced to happen again and again along the play, so that this strategy is well defined. In the end, the infinite word played by Player II is a factorization of the infinite word $b_{0} b_{1} b_{2} \ldots$, and Lemma 1.2 shows that these two infinite words have the same image under the infinite product $\pi_{T}$. Since $\sigma$ is winning for Player II in $\mathbb{S} G\left(X, s^{-1} Y\right)$, the strategy described above is also winning for II in $\overline{\mathbb{S G}}\left(X, s^{-1} Y\right)$. Therefore $X \leq \overline{S G} s^{-1} Y$.

Proposition 2.3. Let $S=\left(S_{+}, S_{\omega}\right)$ be a finite $\omega$-semigroup, and let $X \subseteq S_{\omega}$. The following conditions are equivalent:

(1) $X$ is non-self-dual.

(2) $X \leq \overline{S G} X$.

(3) There exists a prefix $s$ of a linked pair of $S_{+}^{2}$ such that $X \equiv_{S G} s^{-1} X$. 
Proof.

$(2) \Rightarrow(1)$ Given a winning strategy $\sigma$ for Player II in $\overline{\mathbb{S}} \mathbb{G}(X, X)$, we describe a winning strategy for Player I in $\mathbb{S} G\left(X, X^{c}\right)$ : Player I first plays $\sigma(-)$, and then applies $\sigma$ to Player II's moves. He wins.

$(1) \Rightarrow(2)$ Conversely, given a winning strategy $\sigma$ for Player I in $\mathbb{S} \mathbb{G}\left(X, X^{c}\right)$, we describe a winning strategy for Player II in $\overline{\mathbb{S G}}(X, X)$ : she first computes the moves $\sigma(\varepsilon), \sigma(-), \sigma(-,-), \sigma(-,-,-) \ldots$, and plays the first of these elements which is a prefix position. Notice that such a move always exists, since $S_{+}$is finite. From this prefix position, she then applies $\sigma$ to Player I's moves, but restricts herself to playing from one prefix position to another, exactly as described in Lemma 2.2. She wins the game.

$(3) \Rightarrow(2)$ Given any element $s \in S_{+}$, the relation $s^{-1} X \leq_{S G} X$ always holds. Indeed, the winning strategy for Player II consists in first playing $s$, and then copying Player I's moves. The relation $X \equiv_{S G} s^{-1} X$ is thus equivalent to $X \leq_{S G} s^{-1} X$, and Lemma 2.2 ensures that $X \leq_{S G} s^{-1} X$ if and only if $X \leq \overline{S G} s^{-1} X$, for any prefix $s$. Thus, given a prefix $s$ and a winning strategy $\sigma$ for II in $\overline{\mathbb{S G}}\left(X, s^{-1} X\right)$, we describe a winning strategy for II in $\overline{\mathbb{S G}}(X, X)$ : she plays $s$ and then applies $\sigma$.

$(2) \Rightarrow(3)$ Assume that $X \not \equiv_{S G} s^{-1} X$, for every prefix $s$ of $S_{+}$. This means that, for every prefix $s$, Player I has a winning strategy $\sigma_{s}$ in the game $\mathbb{S} \mathbb{G}\left(X, s^{-1} X\right)$. We then describe a winning strategy for Player I in the game $\overline{\mathbb{S G}}(X, X)$ : Player I skips his first move; Player II's answer is forced to be a prefix position $s$, by definition of the $\overline{\mathbb{S} G}$-game; then, Player I applies $\sigma_{s}$, and wins.

Corollary 2.4. Let $S=\left(S_{+}, S_{\omega}\right)$ be a finite $\omega$-semigroup, and let $X \subseteq S_{\omega}$. If $X$ is self-dual, then $X=\bigcup_{s \in I} s Y_{s}$, for some subset $I \subseteq S_{+}$, and some family of non-self-dual $\omega$-subsets $\left(Y_{s}\right)_{s \in I}$ satisfying $Y_{s}<_{S G} X$.

Proof. Let $X \subseteq S_{\omega}$ be self-dual, and let $I$ be the set of prefixes of linked pairs of $S_{+}^{2}$. We observe that $X=\bigcup_{s \in I} s\left(s^{-1} X\right)$. Now, since $X$ is self-dual, Proposition 2.3 ensures that $s^{-1} X<_{S G} X$, for every prefix $s \in I$. Moreover, for every prefix $s \in I$, there exists an idempotent $e$ such that $(s, e)$ is a liked pair. Since $s e=s$, one has $s^{-1} X=(s e)^{-1} X=e^{-1}\left(s^{-1} X\right)$, thus in particular $s^{-1} X \equiv_{S G} e^{-1}\left(s^{-1} X\right)$. Moreover, since $e$ is a prefix of the linked pair $(e, e)$, Proposition 2.3 shows that the set $s^{-1} X$ is non-self-dual, for all $s \in I$. This concludes the proof.

By the previous corollary, the self-dual $\omega$-subsets of finite $\omega$-semigroups can be expressed as finite unions of translations of strictly smaller non-self-dual sets. Hence, in order to exclusively concentrate on the non-self-dual sets, we consider a modified definition of the $\mathbb{S} G$-degree which sticks the self-dual sets to the nonself-dual ones located just one level below it.

$$
d_{s g}(X)= \begin{cases}1 & \text { if } X=\emptyset \text { or } X=\emptyset^{c} \\ \sup \left\{d_{s g}(Y)+1 \mid Y \text { n.s.d. and } Y<_{S G} X\right\} & \text { if } X \text { is non-self-dual, } \\ \sup \left\{d_{s g}(Y) \mid Y \text { n.s.d. and } Y<_{S G} X\right\} & \text { if } X \text { is self-dual. }\end{cases}
$$




\section{Describing the $\mathbb{F} \mathbb{S} G-H i E R A R C h y$}

\subsection{Finite SEMIGROUPS AS GRAPHS}

In this section, we describe a graph representation of finite semigroups by focusing on specific positions in, and moves of the $\mathbb{S} G$-game. The notion of a linked pair is essential to this description. As a consequence, every $\mathbb{S} \mathbb{G}$-play induces a unique path in the graph inherited from the semigroup involved. From this point onward, the set $S_{+}$denotes a fixed finite semigroup. We recall that $P$ and $E$ respectively denote the sets of prefixes and idempotents of $S_{+}$.

Linked pairs satisfy the following game theoretical properties. First of all, Proposition 2.3 shows that any $\mathbb{S} \mathbb{G}$-player in charge of a non-self-dual $\omega$-subset can restrict her/himself to only reaching prefix positions. Also, an $\mathbb{S G}$-player can stay indefinitely in a position $s$ if and only if $s$ is a prefix. S/He does so by playing idempotents in $E(s)$. Finally, for every $s \in P$, each idempotent $e$ of $E(s)$ corresponds to some specific waiting move for the prefix position $s$. These specific positions and moves yield two preorders on the sets of prefixes and idempotents of linked pairs.

Firstly, we consider the restriction of the preorder $\leq_{\mathcal{R}}$ to the set of prefixes $P$, also denoted by $\leq_{\mathcal{R}}$ without ambiguity. By definition, this preorder satisfies the accessibility relation $s \geq_{\mathcal{R}} s^{\prime}$ if and only if there exists $x \in S_{+}^{1}$ such that $s x=s^{\prime}$, for all $s, s^{\prime} \in P$. As usual, one has $s>_{\mathcal{R}} s^{\prime}$ if and only if $s \geq_{\mathcal{R}} s^{\prime}$ and $s^{\prime} \geq_{\mathcal{R}} s$, and also $s \mathcal{R} s^{\prime}$ if and only if $s \geq_{\mathcal{R}} s^{\prime}$ and $s^{\prime} \geq_{\mathcal{R}} s$. This preorder can be naturally extended to the set of $\mathcal{R}$-classes of prefixes $P / \mathcal{R}$ by setting $\bar{s} \geq_{\mathcal{R}} \bar{t}$ if and only if there exist $s^{\prime} \in \bar{s}$ and $t^{\prime} \in \bar{t}$ such that $s^{\prime} \geq_{\mathcal{R}} t^{\prime}$, for all $\bar{s}, \bar{t} \in P / \mathcal{R}$. The pair $\left(P / \mathcal{R}, \geq_{\mathcal{R}}\right)$ is therefore a partial ordering.

Secondly, we consider the natural order on idempotents, denoted by $\leq$, and defined as the restriction of the preorder $\leq_{\mathcal{H}}$ to the set $E$. It satisfies the absorption relation $e \geq e^{\prime}$ if and only if $e e^{\prime}=e^{\prime} e=e^{\prime}$ holds, for all $e, e^{\prime} \in E$. As usual, one has $e>e^{\prime}$ if and only if both $e \geq e^{\prime}$ and $e^{\prime} \nsupseteq e$ hold. The pair $(E, \geq)$ is also a partial ordering [16].

These two relations satisfy the following properties, central in the description of an $\mathbb{S} G$-play. Firstly, a player can move from the prefix position $s$ to the prefix position $s^{\prime}$ if and only if $s \geq_{\mathcal{R}} s^{\prime}$. He can go from $s$ to $s^{\prime}$ and back to $s$ if and only if $s \mathcal{R} s^{\prime}$. Secondly, a player which forever stays in the prefix position $s$ by playing infinitely many $e$ 's and $f$ 's in $E(s)$ produces an infinite play $\alpha$ of the form $(s, e, f, f, e, f, e, e, \ldots)$. If $e \geq f$, since the $f$ 's absorb all the $e$ 's, the infinite word $(s, f, f, f, \ldots)$ is a factorization of $\alpha$, and Lemma 1.2 ensures that $\pi_{S}(\alpha)=s f^{\omega}$. Therefore, only the $\leq$-least idempotents that are played infinitely often in a given prefix position are involved in the final acceptance of the play.

The graph of the preorder $\left(P, \geq_{\mathcal{R}}\right)$ is a subgraph of the right Cayley graph of $S_{+}$, and its strongly connected components are the $\mathcal{R}$-classes of $P$. The graph of the partial order $\left(P / \mathcal{R}, \geq_{\mathcal{R}}\right)$ is thus a directed acyclic graph (DAG) where vertices represent the $\mathcal{R}$-classes of prefixes and directed edges stand for the strict accessibility relation $>_{\mathcal{R}}$, as illustrated in Figure 2, where transitive arrows are 


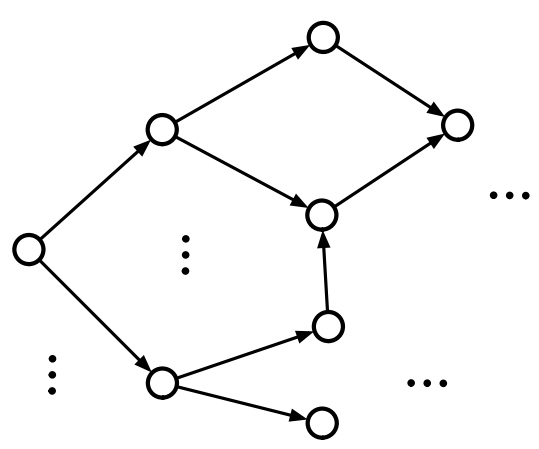

FIGURE 2. The directed acyclic graph representation of the partial order $\left(P / \mathcal{R}, \geq_{\mathcal{R}}\right)$. A play of an $\mathbb{S} \mathbb{G}$-player induces a unique path in this DAG.

not drawn, for reasons of clarity (that is every time there is an edge from $i$ to $j$, and from $j$ to $k$, the induced edge from $i$ to $k$ is dismissed). The successive moves of an $\mathbb{S} G$-player should be traced inside this graph, for every $\mathbb{S} \mathbb{G}$-play according to elements of $S_{+}$induces a sequence of prefix positions which progresses deeper and deeper inside this structure; Therefore, any infinite $\mathbb{S} \mathbb{G}$-play yields a unique path in this DAG that either remains in an $\mathcal{R}$-class of prefixes, or climbs along the edges, with no chance of going back (this justifies the consideration of the partial order $\left(P / \mathcal{R}, \geq_{\mathcal{R}}\right)$ instead of $\left.\left(P / \mathcal{R}, \leq_{\mathcal{R}}\right)\right)$.

Furthermore, every prefix $t$ can be associated with the partial ordered set $(E(t), \geq)$ - called the petal - associated with $t$, and denoted by petal $(t)$. The graph of this set is also a DAG, and given $e, f \in \operatorname{petal}(t)$, there is an edge from $e$ to $f$ if and only if $e>f$. The set petal $(t)$ consists of all the possible waiting moves for the prefix position $t$ ordered by their absorption capacity. Up to making copies of idempotents, we assume all petals to be disjoint. Then, for every $\mathcal{R}$-class of prefixes $\bar{s}$, the set $\bigcup_{t \in \bar{s}} \operatorname{petal}(t)$ will be called the flower associated with $\bar{s}$, denoted by flower $(\bar{s})$. This set contains all the possible waiting moves for some prefix position in $\bar{s}$. Figure 3 illustrates a flower in detail.

The enriched graph representation of $\left(P / \mathcal{R}, \geq_{\mathcal{R}}\right)$ where each $\mathcal{R}$-class of prefixes is associated with its corresponding flower will be called the DAG representation of the finite semigroup $S_{+}$. It can be drawn like a bunch of flowers, as illustrated in Figure 4. This graph acts like an arena for an $\mathbb{S} \mathbb{G}$-player moving in $S_{+}$. It allows to follow the successive prefix positions reached along the play, and for every prefix position, it describes all the possible waiting moves ordered by their absorption capacity.

Finally, we prove that a strictly descending chain of idempotents of length $n+1$ in $S_{+}$implies the existence of $n+1$ distinct accessible growing flowers. 


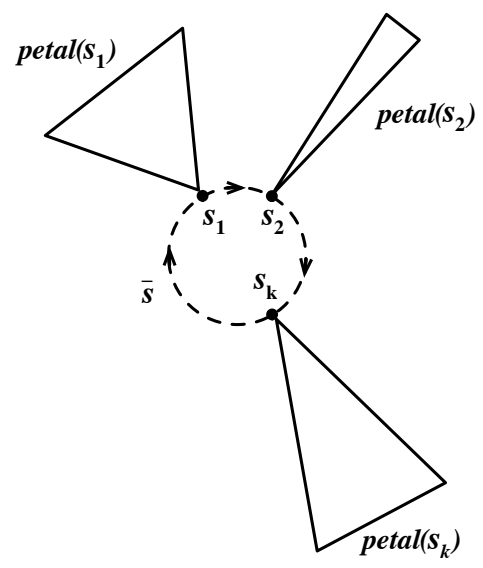

Figure 3 . The set flower $(\bar{s})$ associated with the $\mathcal{R}$-class of prefixes $\bar{s}$. Every prefix $s_{i}$ in $\bar{s}$ is associated with its corresponding petal. The circle describes the $\geq_{\mathcal{R}}$-accessibility relation between the prefixes $s_{i}$ of $\bar{s}$.

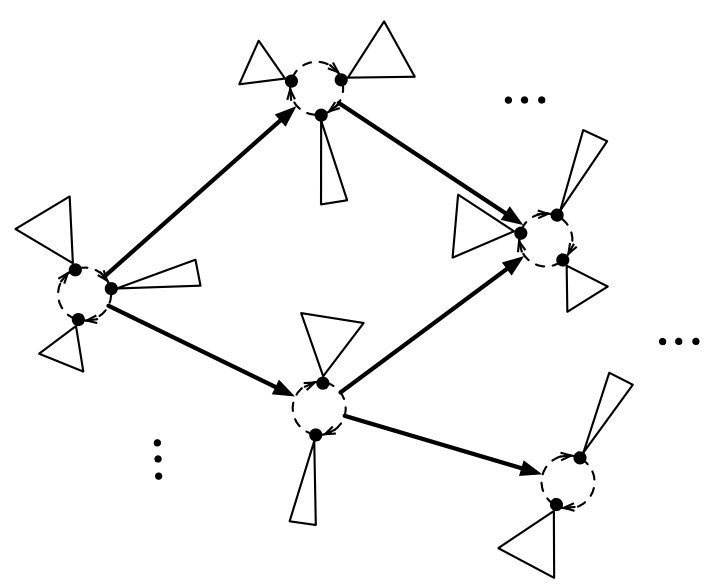

FIGURE 4. The DAG representation of a finite semigroup $S_{+}$: every $\mathcal{R}$-class of prefixes is associated with its corresponding flower. This DAG is an arena for every $\mathbb{S} G$-player moving inside the semigroup $S_{+}$.

Proposition 3.1. Let $e_{0}>e_{1}>\ldots>e_{n}$ be any strictly descending chain of idempotents in $S_{+}$. Then the DAG representation of $S_{+}$contains the flowers flower $\left(\bar{e}_{0}\right)$, flower $\left(\bar{e}_{1}\right), \ldots$, flower $\left(\bar{e}_{n}\right)$ such that:

- $\bar{e}_{i}$ is the $\mathcal{R}$-class of prefixes of $e_{i}$, for all $i \leq n$; 

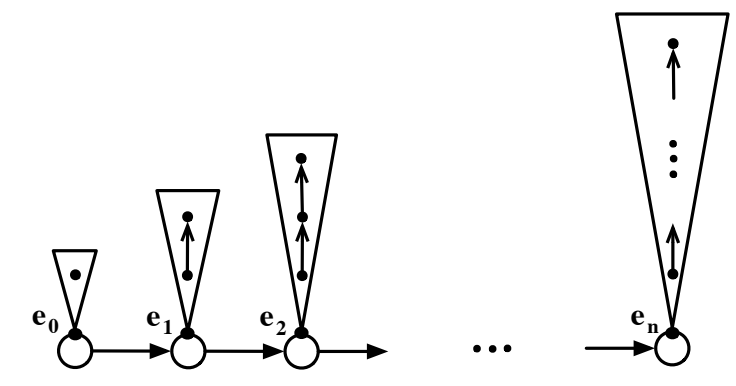

Figure 5. A chain of idempotents $e_{0}>e_{1}>\ldots>e_{n}$ ensures the existence of a linear sequence of $n+1$ distinct growing flowers.

- $\bar{e}_{i}>_{\mathcal{R}} \bar{e}_{j}$ whenever $i<j$;

- flower $\left(\bar{e}_{i}\right)$ contains the chain of idempotents $e_{0}>\ldots>e_{i}$, for all $i \leq n$, as illustrated in Figure 5.

Proof. For each idempotent $e$, the pair $(e, e)$ is obviously linked, hence every idempotent $e$ is also a prefix. Therefore, the DAG representation of $S_{+}$contains the following $n+1$ flowers

$$
\text { flower }\left(\bar{e}_{0}\right) \text {, flower }\left(\bar{e}_{1}\right), \ldots, \text { flower }\left(\bar{e}_{n}\right) \text {, }
$$

where each $\bar{e}_{i}$ denotes the $\mathcal{R}$-class of $e_{i}$. Moreover, the relation $e_{i}>e_{j}$ implies $e_{i}>_{\mathcal{R}} e_{j}$, for every $i<j$. Finally, one has $e_{i} e_{k}=e_{i}$, for every $k \leq i$, therefore the chain $e_{0}>\ldots>e_{i}$ is contained in flower $\left(\bar{e}_{i}\right)$, for all $i \leq n$.

\subsection{Finite Pointed $\omega$-Semigroups AS GRAPhS}

The DAG representation of finite semigroups can be extended to some graph representation of finite pointed $\omega$-semigroups. For that purpose, we introduce the signature of a petal. From this point onward, the pair $(S, X)$ denotes a fixed finite pointed $\omega$-semigroup, where $S=\left(S_{+}, S_{\omega}\right)$ is a finite $\omega$-semigroup and $X$ is a subset of $S_{\omega}$.

Definition 3.2. Let $s \in P$. The signature of the set petal $(s)$ according to $X$ is the mapping $\operatorname{sign}_{X}: \operatorname{petal}(s) \longrightarrow\{+,-\}$ defined by

$$
\operatorname{sign}_{X}(e)= \begin{cases}+ & \text { if } s e^{\omega} \in X, \\ - & \text { if } s e^{\omega} \notin X .\end{cases}
$$

The pair $\left(\operatorname{petal}(s), \operatorname{sign}_{X}\right)$ is called the signed petal associated with $s$, denoted by $\operatorname{petal}_{X}(s)$. The union for $t$ running in $\bar{s}$ of the sets $\operatorname{petal}_{X}(t)$ is called the signed flower associated with $\bar{s}$, denoted by flower ${ }_{X}(\bar{s})$. 


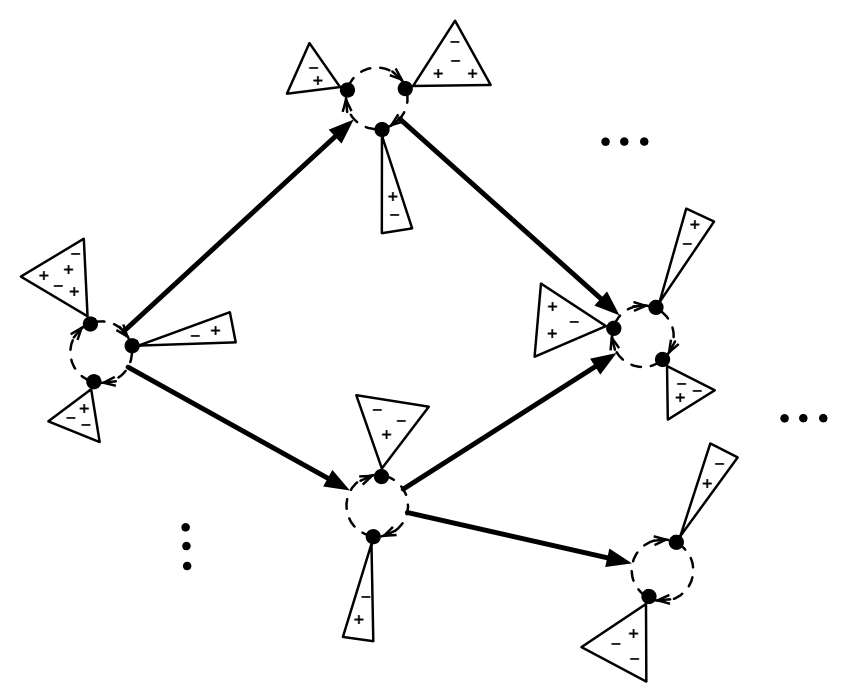

Figure 6 . The signed DAG representation of a finite pointed $\omega$ semigroup $(S, X)$ : an enriched arena for an $\mathbb{S} \mathbb{G}$-player in charge of $X$.

The graph of the partial order $\left(P / \mathcal{R}, \geq_{\mathcal{R}}\right)$ where each $\mathcal{R}$-class of prefixes $\bar{s}$ is associated with its corresponding signed flower - flower $_{X}(\bar{s})$ - is called the signed $D A G$ representation of the finite pointed $\omega$-semigroup $(S, X)$, and is illustrated in Figure 6. This graph is an arena for an $\mathbb{S} G$-player in charge of $X$ : the successive prefix positions reached along the play can be traced inside this graph, just as described in Section 3.1. But in addition, the signs associated with the idempotents provide information about the acceptance of an $\mathbb{S} G$-play according to $X$ : an infinite play belongs to $X$ if and only if it can be factorized into the form $s e^{\omega}$, for some positive $e \in \operatorname{petal}_{X}(s)$. Finally, by finiteness of this DAG, every infinite play will eventually remain forever in a signed flower, and hit at least one of the corresponding signed petals infinitely often.

Example 3.3. Let $S=\left(\{0,1\},\left\{0^{\omega}, 1^{\omega}\right\}\right)$ be the finite $\omega$-semigroup defined by the following relations:

$$
\begin{array}{llll}
0 \cdot 0=0 & 0 \cdot 1=0 & 1 \cdot 0=0 & 1 \cdot 1=1 \\
00^{\omega}=0^{\omega} & 10^{\omega}=0^{\omega} & 01^{\omega}=1^{\omega} & 11^{\omega}=1^{\omega} .
\end{array}
$$

Let $X=\left\{0^{\omega}\right\} \subseteq S$. The signed DAG representation of $(S, X)$ is illustrated in Figure 7 . 


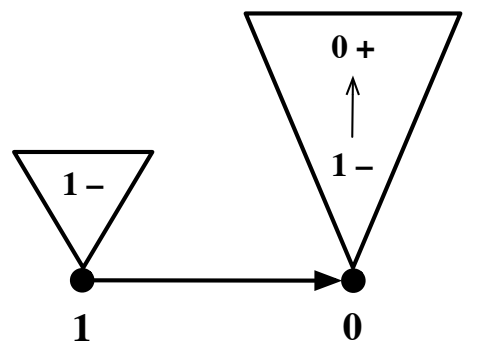

Figure 7. The signed DAG representation of $(S, X)$.

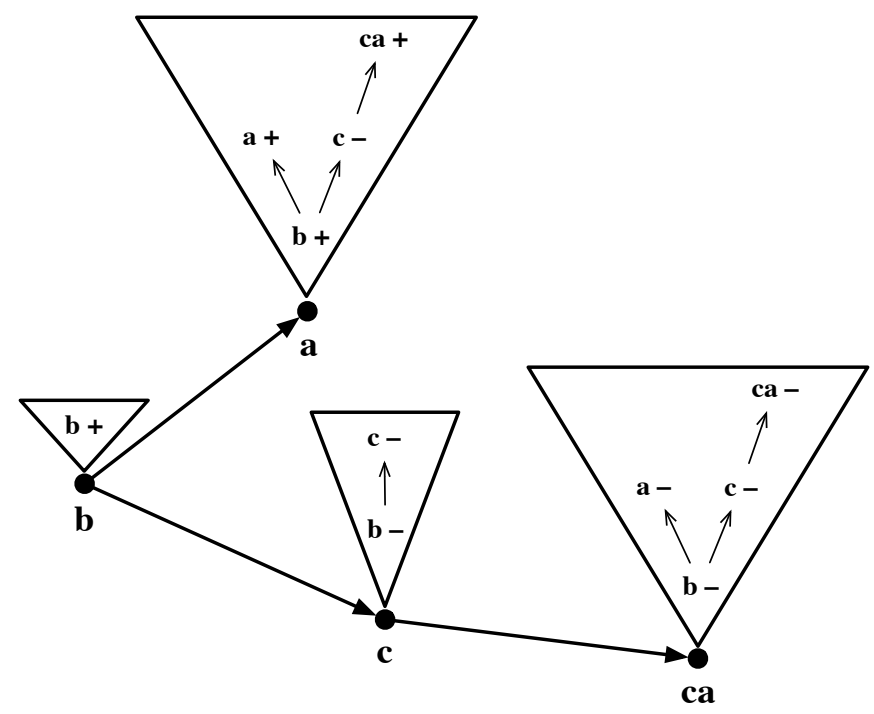

FigURE 8. The signed DAG representation of $(T, Y)$.

Example 3.4. Let $T=\left(\{a, b, c, c a\},\left\{a^{\omega},(c a)^{\omega}, 0\right\}\right)$ be the finite $\omega$-semigroup defined by the following relations:

$$
\begin{aligned}
& a^{2}=a \quad a b=a \quad a c=a \quad b a=a \\
& b^{2}=b \quad b c=c \quad c b=c \quad c^{2}=c \\
& b^{\omega}=a^{\omega} \quad c^{\omega}=0 \quad a a^{\omega}=a^{\omega} \quad a(c a)^{\omega}=a^{\omega} \\
& b a^{\omega}=a^{\omega} \quad b(c a)^{\omega}=(c a)^{\omega} \quad c a^{\omega}=(c a)^{\omega} \quad c(c a)^{\omega}=(c a)^{\omega} .
\end{aligned}
$$

Let $Y=\left\{a^{\omega}\right\} \subseteq T$. The signed DAG representation of $(T, Y)$ is illustrated in Figure 8. 


\subsection{Alternating Chains}

The following sections describe, step by step, the relevant game theoretical characteristics of the signed DAG representation of a finite pointed $\omega$-semigroup. For that purpose, we introduce the notion of an alternating chain of idempotents in a signed petal. This definition refines the notion of a chain in finite $\omega$-semigroups, introduced in [3], Theorem 6.

Definition 3.5. Let $s \in P$. An alternating chain in $\operatorname{petal}_{X}(s)$ is a strictly descending sequence of idempotents of $\operatorname{petal}_{X}(s) e_{0}>e_{1}>\ldots>e_{n}$ satisfying the following properties:

(1) signs alternation: one has $\operatorname{sign}_{X}\left(e_{k}\right) \neq \operatorname{sign}_{X}\left(e_{k+1}\right)$, for all $k<n$;

(2) each $e_{k}$ is minimal for its sign: if $e_{k}>e$ and $\operatorname{sign}_{X}\left(e_{k}\right)=\operatorname{sign}_{X}(e)$, then there exists $f$ such that $e_{k}>f>e$ and $\operatorname{sign}_{X}\left(e_{k}\right) \neq \operatorname{sign}_{X}(f)$.

An alternating chain in a signed flower is simply an alternating chain in a signed petal of this signed flower.

Let $C: e_{0}>e_{1}>\ldots>e_{n}$ be an alternating chain in $\operatorname{petal}_{X}(s)$. The length of $C$, denoted by $l(C)$, is $n$ (number of its elements minus one, or equivalently, the number of signs alternations). The chain $C$ is said to be maximal in $\operatorname{petal}_{X}(s)$ if there is no other alternating chain of strictly larger length in $\operatorname{petal}_{X}(s)$. Maximal alternating chains in signed petals and flowers will play a central role in the sequel. In addition, the chain $C$ is called positive if $\operatorname{sign}_{X}\left(e_{0}\right)=+$, and negative otherwise. Two alternating chains $e_{0}>\ldots>e_{n}$ and $e_{0}^{\prime}>\ldots>e_{n}^{\prime}$ of the same length are said to have the same signs if $\operatorname{sign}_{X}\left(e_{n}\right)=\operatorname{sign}_{X}\left(e_{n}^{\prime}\right)$, and opposite signs otherwise. Condition (1) of Definition 3.5 implies that these chains have the same signs if and only if $\operatorname{sign}_{X}\left(e_{i}\right)=\operatorname{sign}_{X}\left(e_{i}^{\prime}\right)$, for all $i$. Finally, we say that an alternating chain $C$ captures the idempotent $e$ if $e \geq e_{0}$, or if there exist $e_{i}$ and $e_{i+1}$ such that

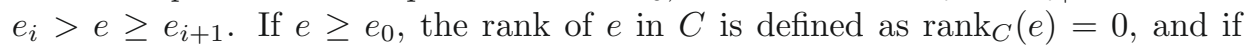
$e_{i}>e \geq e_{i+1}$, then $\operatorname{rank}_{C}(e)=i+1$. An alternating chain of length 3 capturing the elements $e$ and $e^{\prime}$ is illustrated below. Every idempotent is associated with its sign; arrows represent the $>$-relation.

$$
\left(e_{0},+\right) \longrightarrow\left(e_{1},-\right) \rightarrow(e,+) \rightarrow\left(e_{2},+\right) \rightarrow\left(e^{\prime},-\right) \rightarrow\left(e_{3},-\right)
$$

Example 3.6. Consider the finite pointed $\omega$-semigroup $(T, Y)$ given in Example 3.4. The sequence $b>c>c a$ is a positive alternating chain of length 2 in the signed petal petal ${ }_{Y}(a)$. Inside the signed petal petal ${ }_{Y}(c a)$, the element $c a$ is a negative alternating chain of length 0 capturing the idempotents $b$ and $c$.

Alternating chains satisfy the following property.

Lemma 3.7. Let $x \in \operatorname{petal}_{X}(s)$. Among all the longest alternating chains capturing $x$, any two bear the same signs, hence induce the same rank for $x$.

Consequently, we simply denote by $\operatorname{rank}(e)$ the rank of $e$ in any longest alternating chain capturing $e$. 
Proof. Let $C_{1}: e_{0}>\ldots>e_{n}$ and $C_{2}: f_{0}>\ldots>f_{n}$ be any two of the longest alternating chains capturing $x$. We prove that their $\leq$-minimal elements $e_{n}$ and $f_{n}$ have the same sign. Consider $e=\left(e_{n} f_{n} e_{n}\right)^{\pi}$ and $f=\left(f_{n} e_{n} f_{n}\right)^{\pi}$, where $\pi$ is the exponent of $S_{+}$. Then $e$ and $f$ are idempotent and $s e=s f=s$, hence $e$ and $f$ both belong to $\operatorname{petal}_{X}(s)$. Moreover, $e_{n} e=e e_{n}=e$, thus $e_{n} \geq e$. Since $C_{1}$ is a longest alternating chain capturing $x$, and $e_{n}$ is minimal in this chain, the elements $e$ and $e_{n}$ have the same sign. Condition (2) of Definition 3.5 then implies that $e_{n}=e$. Similarly, $f_{n}=f$. Hence, the properties of the $\omega$-operation imply

$$
s e^{\omega}=s\left(e_{n} f_{n} e_{n}\right)^{\omega}=s\left(e_{n} f_{n} f_{n} e_{n}\right)^{\omega}=s e_{n} f_{n}\left(f_{n} e_{n} e_{n} f_{n}\right)^{\omega}=s\left(f_{n} e_{n} f_{n}\right)^{\omega}=s f^{\omega} .
$$

Therefore, the idempotents $e=e_{n}$ and $f=f_{n}$ have the same sign, hence $C_{1}$ and $C_{2}$ also have the same signs. We now prove that $x$ has the same rank in $C_{1}$ and $C_{2}$. Let $k$ and $l$ be the respective ranks of $x$ in $C_{1}$ and $C_{2}$. We may assume, without loss of generality, that $k \leq l$. Therefore,

$$
\begin{gathered}
e_{0}>e_{1}>\ldots>e_{k-1}>f_{\ell}>\ldots>f_{n}, \\
f_{0}>f_{1}>\ldots>f_{\ell-1}>e_{k}>\ldots>e_{n}
\end{gathered}
$$

are two alternating chains of respective lengths $(k-1)+(n-l)+1=k+(n-l)$ and $(l-1)+(n-k)+1=l+(n-k)$. The maximality of $n$ implies both $k+(n-l) \leq n$ and $l+(n-k) \leq n$, thence $k=l$.

\subsection{VEINS}

We now focus on some specific alternating chains of idempotents called veins. We prove that only these influence the $\mathbb{S} G$-degree of our algebraic structures.

Definition 3.8. For every $s$ in $P$, a maximal alternating chain in $\operatorname{petal}_{X}(s)$ is called a vein of this signed petal.

Example 3.9. Consider the finite pointed $\omega$-semigroup $(T, Y)$ given in Example 3.4. The sequence $b>c>c a$ is a vein $\operatorname{in}_{\operatorname{petal}_{Y}}(a)$.

Playing waiting moves inside a given vein instead of potentially being able to play through all idempotents of a signed petal will show not to be restricting. We first prove the following property.

Lemma 3.10. Any two veins of a given signed petal share the same signs.

Proof. Let $C_{1}$ and $C_{2}$ be two veins inside $\operatorname{petal}_{X}(s)$. As mentioned in the proof of Lemma 3.7, the respective $\leq$-minimal elements $m_{1}$ and $m_{2}$ of $C_{1}$ and $C_{2}$ have the same sign. Therefore $C_{1}$ and $C_{2}$ share the same signs too.

We now define a mapping from any signed petal onto one of its veins. The choice of the vein may be arbitrary, for Lemma 3.10 shows that all the veins of a given signed petal are isomorphic. This mapping will be involved in the strategy of an $\mathbb{S G}$-player restricting his waiting moves to the sole idempotents of such veins. 


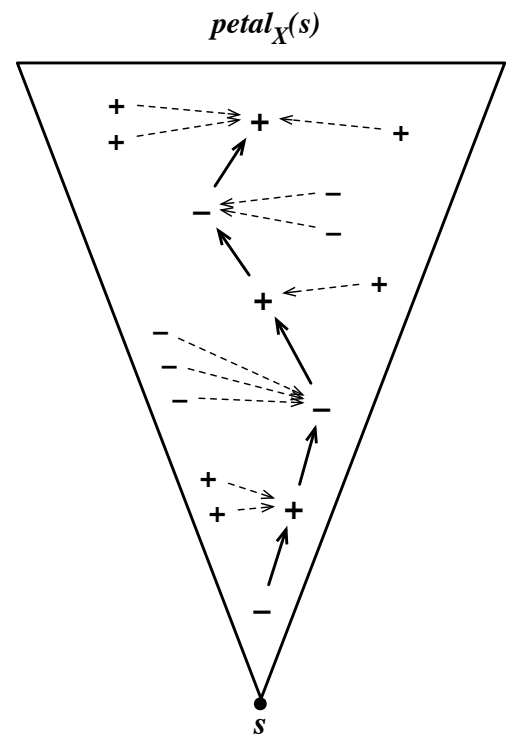

FiguRE 9. The surjection from a signed petal onto one of its veins.

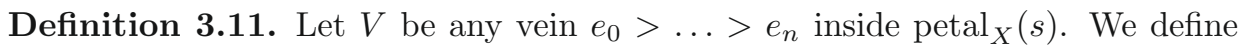
the mapping $\sigma: \operatorname{petal}_{X}(s) \longrightarrow V$ by

$$
\sigma(e)= \begin{cases}e_{i} & \text { if } \operatorname{rank}(e)=i \text { and } \operatorname{sign}_{X}(e)=\operatorname{sign}_{X}\left(e_{i}\right), \\ e_{i+1} & \text { if } \operatorname{rank}(e)=i \text { and } \operatorname{sign}_{X}(e) \neq \operatorname{sign}_{X}\left(e_{i}\right)\end{cases}
$$

By finiteness of the set $\operatorname{petal}_{X}(s)$, this mapping is effectively computable. It is onto and preserves the order $\leq$ as well as the signature, as illustrated in Figure 9.

We finally come to prove that only one vein of each signed petal is significant in the computation of the $\mathbb{S} \mathbb{G}$-degree of $(S, X)$. More precisely, we show that any $\mathbb{S} \mathbb{G}$ player remaining indefinitely in some prefix position $s$ can restrict her/his waiting moves to the idempotents of a given vein of $\operatorname{petal}_{X}(s)$. To this end, we consider the imposed version of the game $\mathbb{S} G(X, X)$ where:

- both players are in charge of $X$, and are not allowed to pass their turns;

- they are both forced to play $s$ on their first move;

- on his next moves, I is forced to play waiting moves inside $\operatorname{petal}_{X}(s)$;

- on her next moves, II is forced to play waiting moves belonging exclusively to a given vein of $\operatorname{petal}_{X}(s)$.

We prove that these restricted rules for Player II do actually not weaken her.

Proposition 3.12. Player II has a winning strategy in the above restricted game. 
Proof. Both players are forced to play $s$ on their first move. A winning strategy for Player II is described by induction as follows.

Strategy. Player II first associates with each element $e$ in $\operatorname{petal}_{X}(s)$ a counter $\kappa(e)$. After each move of I, the integer $\kappa(e)$ will be the largest possible number of $e$ 's occurring in a factorization of I's current play. More precisely, Player II updates these counters as follows: let $\left(e_{0}, \ldots, e_{k-1}\right)$ be the elements of $\operatorname{petal}_{X}(s)$ already played by $\mathrm{I}$, then for each $e$ in $\operatorname{petal}_{X}(s)$, the value of $\kappa(e)$ is set as the largest integer $p$ such that there exists a sequence of indices

$$
0 \leq i_{1} \leq j_{1}<i_{2} \leq j_{2}<\ldots<i_{p} \leq j_{p} \leq k-1
$$

satisfying $e=\left(e_{i_{1}} \cdots e_{j_{1}}\right)=\left(e_{i_{2}} \cdots e_{j_{2}}\right)=\ldots=\left(e_{i_{p}} \cdots e_{j_{p}}\right)$. After that, Player II computes the images on the given vein under $\sigma$ of all the idempotents whose counters has increased, as described in Definition 3.11. She finally plays the $\leq-$ minimum of these images. Notice that this minimum always exists since the given vein is well ordered by $\leq$.

The following three claims prove that this strategy is winning for Player II. We first set $i n c_{\infty}$ for the set of idempotents of $\operatorname{petal}_{X}(s)$ whose counters were incremented infinitely often during the play, and we let $I N C_{\infty}$ be the set of $\leq$ minimal elements of $i n c_{\infty}$. Finally, we set

$$
e_{\min }=\min \left\{\sigma(e) \mid e \in I N C_{\infty}\right\} .
$$

Claim 3.13. Let $\alpha$ be I's infinite play, and let $e \in I N C_{\infty}$. Then $\pi_{S}(\alpha)=s e^{\omega}$.

Proof. Since $e$ belongs to $I N C_{\infty}$, its counter was incremented infinitely often during the play. Consequently, I's infinite play can be written as

$$
\alpha=s v_{0} e v_{1} e v_{2} e v_{3} e v_{4} e \ldots,
$$

where each $v_{i}$ is a finite word of $\operatorname{petal}_{X}(s)^{*}$, for all $i \geq 0$. By idempotence of $e$, the infinite word $\alpha$ is a factorization of $\beta=s v_{0} e v_{1} e e v_{2} e e v_{3} e e v_{4} e e . .$. , and the infinite word $\gamma=s v_{0}\left(e v_{1} e\right)\left(e v_{2} e\right)\left(e v_{3} e\right) \cdots$ is a factorization of $\beta$. By Proposition 1.1, $\gamma$ can be associated with a linked pair $(s, \tilde{e})$, where $\tilde{e}=e v e$, for some $v \in \operatorname{petal}_{X}(s)^{*}$. Thus $\pi_{S}(\gamma)=s \tilde{e}^{\omega}$. Moreover, by Lemma 1.2, since $\gamma$ is a factorization of $\beta$, one has $\pi_{S}(\gamma)=\pi_{S}(\beta)=s \tilde{e}^{\omega}$. Also, since $\alpha$ is a factorization of $\beta$, then $\pi_{S}(\alpha)=$ $\pi_{S}(\beta)=s \tilde{e}^{\omega}$. Besides, notice that the element $\tilde{e}$ also appears infinitely often in a factorization of $\alpha$, hence its counter was incremented infinitely often during the play, meaning that $\tilde{e} \in i n c_{\infty}$. In addition, one has $e \tilde{e}=\tilde{e} e=\tilde{e}$, thus $e \geq \tilde{e}$. But then the minimality of $e$ in $i n c_{\infty}$ implies $\tilde{e}=e$. Finally, one obtains $\pi_{S}(\alpha)=$ $s \tilde{e}^{\omega}=s e^{\omega}$.

Claim 3.14. Let $\beta$ be II's infinite play. Then $\pi_{S}(\beta)=s e_{\min }{ }^{\omega}$.

Proof. Let $e \in I N C_{\infty}$ such that $e_{\min }=\sigma(e)$. The strategy described above guarantees that II played $e_{\min }$ infinitely often. Therefore, II's infinite play can be 
written as

$$
\beta=s u_{0} e_{\min } u_{1} e_{\min } u_{2} e_{\min } \ldots,
$$

where each $u_{i}$ is a finite word of elements of the given vein, for all $i \geq 0$. Moreover, no element $g<e_{\min }$ was played by II infinitely often. Otherwise, since the set $\sigma^{-1}(g)$ is finite, there would exist $f$ in $i n c_{\infty}$ such that $\sigma(f)=g$, contradicting the minimality of $e_{\min }$. Now, since $e_{\min }$ is the $\leq-$ minimal element of the given vein played infinitely often by II, every product $e_{\min } u_{i}$ is equal to $e_{\min }$. Proposition 1.1 then shows that the infinite word $\beta$ can be associated with the linked pair $\left(s, e_{\min }\right)$. Therefore $\pi_{S}(\beta)=s e_{\min }{ }^{\omega}$.

Claim 3.15. One has $\pi_{S}(\alpha) \in X$ if and only if $\pi_{S}(\beta) \in X$.

Proof. Claim 3.14 shows that $\pi_{S}(\beta)=s e_{\min }{ }^{\omega}$. Now, let $e$ be an idempotent of $I N C_{\infty}$ such that $\sigma(e)=e_{\min }$. Claim 3.13 proves that $\pi_{S}(\alpha)=s e^{\omega}$. Moreover, since $\sigma$ preserves the signature, the idempotents $e$ and $e_{\min }$ have the same sign. Therefore, $\pi_{S}(\alpha)=s e^{\omega} \in X$ if and only if $\pi_{S}(\beta)=s e_{\min }{ }^{\omega} \in X$.

\subsection{MAIN Veins}

In this section, we prove that only some specific veins of each flower is relevant in the computation of the $\mathbb{S} \mathbb{G}$-degree. We focus on these main veins.

Definition 3.16. Let $\bar{s} \in P / \mathcal{R}$. A maximal alternating chain in $\operatorname{flower}_{X}(\bar{s})$ is called a main vein of this signed flower.

Example 3.17. Consider the finite pointed $\omega$-semigroup $(T, Y)$ given in Example 3.4. The sequence $b>c>c a$ is a main vein in $\operatorname{flower}_{Y}(a)$.

Main veins satisfy the same property as veins.

Lemma 3.18. Any two main veins of a given signed flower share the same signs.

Proof. Let $C_{1} \subseteq \operatorname{petal}_{X}\left(s_{1}\right)$ and $C_{2} \subseteq \operatorname{petal}_{X}\left(s_{2}\right)$ be two main veins of flower $X(\bar{s})$. Once again, we prove that their $\leq$-minimal elements $m_{1}$ and $m_{2}$ have the same sign. Since $s_{1}, s_{2} \in \bar{s}$, there exist $a, b \in S_{+}^{1}$ such that $s_{1} a=s_{2}$ and $s_{2} b=s_{1}$. Now, consider the elements $e_{1}=\left(m_{1} a m_{2} b m_{1}\right)^{\pi}$ and $e_{2}=\left(m_{2} b m_{1} a m_{2}\right)^{\pi}$, where $\pi$ is the exponent of $S_{+}$. Exactly as proved in the proof of Lemma 3.7, one has $m_{1}=e_{1}$ and $m_{2}=e_{2}$. Moreover, the properties of the $\omega$-operation ensure that $s_{1} e_{1}{ }^{\omega}=s_{2} e_{2}{ }^{\omega}$. Therefore, $e_{1}=m_{1}$ and $e_{2}=m_{2}$ have the same sign, which proves that $C_{1}$ and $C_{2}$ have the same signs too.

As previously, we define a mapping from every signed petals of a signed flower onto a given main vein. The choice of the main vein may also be arbitrary, for Lemma 3.18 proves that mains veins of a given signed flower are all isomorphic. We implicitly proceed in two steps: firstly, we map every signed petal onto one of 


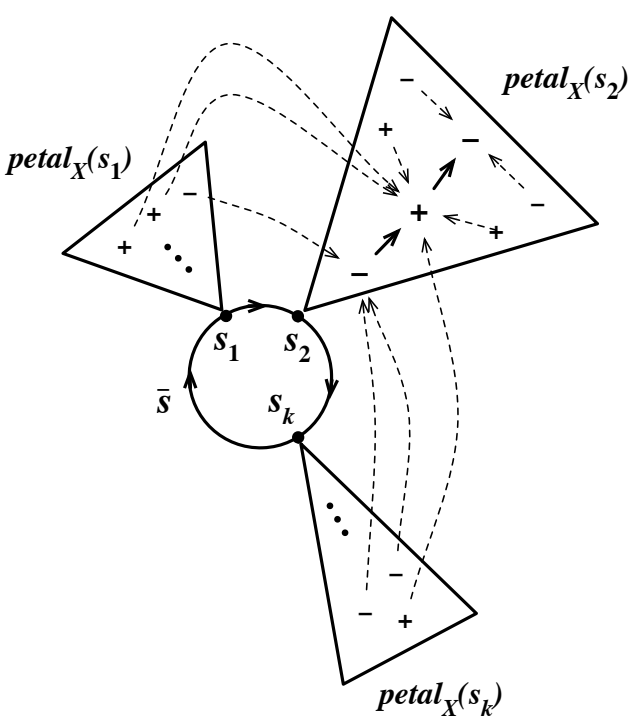

Figure 10. The surjection from a signed flower onto one of its main veins.

its veins, as defined in Definition 3.11; secondly, we map every such vein onto a given main vein.

Definition 3.19. Let $V: e_{0}>\ldots>e_{n}$ be a main vein of $\operatorname{flower}_{X}(\bar{s})$. We define the mapping $\bar{\sigma}$ : flower $_{X}(\bar{s}) \longrightarrow V$ by

$$
\bar{\sigma}(e)= \begin{cases}e_{i} & \text { if } \operatorname{rank}(\sigma(e))=i \text { and } \operatorname{sign}_{X}(e)=\operatorname{sign}_{X}\left(e_{i}\right), \\ e_{i+1} & \text { if } \operatorname{rank}(\sigma(e))=i \text { and } \operatorname{sign}_{X}(e) \neq \operatorname{sign}_{X}\left(e_{i}\right) .\end{cases}
$$

This mapping is onto, and preserves the natural ordering on idempotents, as well as the signature. It is illustrated in Figure 10.

We now show that only one main vein of each signed flower matters in the computation of the $\mathbb{S} \mathbb{G}$-degree of $(S, X)$. In other words, any player remaining indefinitely in some $\mathcal{R}$-class of prefixes $\bar{s}$ can restrict his waiting moves to the idempotents of a given main vein inside flower $_{X}(\bar{s})$. We thence consider a given main vein of flower $_{X}(\bar{s})$ contained in $\operatorname{petal}_{X}(t)$, for some $t \in \bar{s}$, and we introduce an imposed version of the game $\mathbb{S} G(X, X)$ where:

- both players are in charge of $X$, and cannot skip their turns;

- I is forced to only reach positions in $\bar{s}$;

- II is forced to play $t$ on her first move, and then restrict her waiting moves to the idempotents of the given main vein in $\operatorname{petal}_{X}(t)$.

We extend Proposition 3.12 to main veins.

Proposition 3.20. Player II has a winning strategy in this imposed game. 
Proof. Player II fist plays $t$, then applies the following strategy.

Strategy. She associates with each element $e$ in flower $_{X}(\bar{s})$ a counter $\kappa(e)$. She updates these counters after each move of I as follows: let $\left(x_{0}, \ldots, x_{k-1}\right)$ be the elements already played by I, then for every $t^{\prime} \in \bar{s}$ and every $e \in \operatorname{petal}_{X}\left(t^{\prime}\right)$, the value $\kappa(e)$ is the maximal number of occurrences of $e$ appearing in position $t^{\prime}$ in a factorization of I's current play. More precisely, the value of $\kappa(e)$ is set as the largest integer $p$ such that there exists a sequence of indices

$$
0 \leq i_{1} \leq j_{1}<i_{2} \leq j_{2}<\ldots<i_{p} \leq j_{p} \leq k-1
$$

satisfying

(1) $e=\left(x_{i_{1}} \cdots x_{j_{1}}\right)=\left(x_{i_{2}} \cdots x_{j_{2}}\right)=\ldots=\left(x_{i_{p}} \cdots x_{j_{p}}\right)$;

(2) all the elements $x_{i_{1}}, x_{i_{2}}, \ldots, x_{i_{p}}$ were played in position $t$.

Then II computes the images on the given main vein under $\bar{\sigma}$ of all idempotents whose counters were incremented, and plays the $\leq$-minimum of those. If no element were incremented, II plays the $\leq$-largest idempotent of the given main vein. This may happen, for instance, when I passes from one prefix of the $\mathcal{R}$-class to another, and hence doesn't play an idempotent of flower $_{X}(\bar{s})$.

This strategy ensures that Player II increments the counter of an idempotent $e \in \operatorname{petal}_{X}\left(t^{\prime}\right)$ if and only if $e$ appears in position $t^{\prime}$ in a factorization of I's play. The three following claims prove that this strategy is winning for Player II. We first introduce the following notations: we let $i n c_{\infty}$ be the set of elements in flower $_{X}(\bar{s})$ whose counters were incremented infinitely often during the play, and $I N C_{\infty}$ be the set of $\leq$-minimal elements of $i n c_{\infty}$. We also set

$$
e_{\min }=\min \left\{\bar{\sigma}(e) \mid e \in I N C_{\infty}\right\} .
$$

Claim 3.21. Let $\alpha$ be I's infinite play, let $e \in I N C_{\infty}$, and let $r \in \bar{s}$ be such that $e \in \operatorname{petal}_{X}(r)$. Then $\pi_{S}(\alpha)=r e^{\omega}$.

Proof. This proof is very similar to the proof of Claim 3.13. Since the idempotent $e \in \operatorname{petal}_{X}(r)$ has been played infinitely often in position $r$ by Player I, the infinite word $\alpha$ can be associated with a liked pair $(r, \tilde{e})$, where $\tilde{e}$ is an element of $\operatorname{petal}_{X}(r)$ necessarily of the form $\tilde{e}=e v e$, for some $v \in S_{+}^{*}$. It follows that $\tilde{e}=e$, and thus $\pi_{S}(\alpha)=r \tilde{e}^{\omega}=r e^{\omega}$.

Claim 3.22. Let $\beta$ be II's infinite play. Then $\pi_{S}(\beta)=t e_{\min }{ }^{\omega}$ (where $t$ is the prefix associated with the given main vein).

Proof. This proof is very similar to the proof of Claim 3.14. Since there is a finite number of petals in flower $_{X}(\bar{t})$, and since every petal is finite, then no element $g<e_{\min }$ has been played infinitely often by Player II. Therefore, the infinite word $\beta$ can be associated with the linked pair $\left(t, e_{\min }\right)$, thence $\pi_{S}(\beta)=t e_{\min }{ }^{\omega}$.

Claim 3.23. One has $\pi_{S}(\alpha) \in X$ if and only if $\pi_{S}(\beta) \in X$. 
Proof. Claim 3.22 shows that $\pi_{S}(\beta)=t e_{\min }{ }^{\omega}$. Now, let $e \in I N C_{\infty}$ such that $e_{\text {min }}=\bar{\sigma}(e)$, and let $r$ be the prefix such that $e \in \operatorname{petal}_{X}(r)$. Claim 3.21 proves that $\pi_{S}(\alpha)=r e^{\omega}$. Finally, since $\bar{\sigma}$ preserves the signature, the elements $e$ and $e_{\min }$ have the same sign. Therefore, $\pi_{S}(\alpha)=r e^{\omega} \in X$ if and only if $\pi_{S}(\beta)=t e_{\min }{ }^{\omega} \in X$.

\subsection{DAG OF MAIN VEINS}

We now prove that the $\mathbb{S} G$-degree of $(S, X)$ only depends on the structure of the partial ordered set $\left(P / \mathcal{R}, \geq_{\mathcal{R}}\right)$, and on the lengths of the main veins. Consequently, we shall prune the signed DAG representation of $(S, X)$ by focusing specifically on these two graphical features.

As a direct consequence of Proposition 3.20, we prove that an $\mathbb{S G}$-player can restrict all his waiting moves to the idempotents of some given main veins. For this purpose, we consider once again an imposed version of the game $\mathbb{S} G(X, X)$ where:

- both players are in charge of $X$, and cannot skip their turns;

- I plays without restriction, exactly like in a regular $\mathbb{S G}$-game;

- II is allowed to play without restriction while moving from one prefix position to another; however, every prefix position $s$ that she reaches must be such that $\operatorname{petal}_{X}(s)$ contains a main vein $V(\bar{s})$ of flower $_{X}(\bar{s})$, and as long as she remains in such a position $s$, she is forced to play waiting moves inside $V(\bar{s})$.

Proposition 3.24. Player II has a winning strategy in this imposed game.

Proof. Player II follows Player I as described hereafter: every time I reaches an $\mathcal{R}$-class of prefixes $\bar{s}$, Player II reaches a prefix $s_{i}$ of this same $\mathcal{R}$-class $\bar{s}$ such that $\operatorname{petal}_{X}\left(s_{i}\right)$ contains a main vein $V$ of flower $_{X}(\bar{s})$. Then, as long as I's play remains in $\bar{s}$, II plays idempotents of $V$ as described in Proposition 3.20. And so on and so forth. We prove that this strategy is winning for II. By finiteness of the partial ordering $\left(P / \mathcal{R}, \geq_{\mathcal{R}}\right)$, Player I is forced to eventually reach an $\mathcal{R}$-class of prefixes $\bar{s}$ inside which he will remain indefinitely. Thence Player II reaches the prefix $s_{k}$ associated with a given main vein of flower $_{X}(\bar{s})$, and plays until the end of the play as described in Proposition 3.20. She thus wins the game.

Proposition 3.24 ensures that only one main vein of each signed flower matters in the computation of the $\mathbb{S} \mathbb{G}$-degree. Therefore, the signed DAG representation of a finite pointed $\omega$-semigroup can be simplified by deleting all the signed flowers, but only keeping a single main vein for each, as illustrated in Figure 11. Vertices denote the $\mathcal{R}$-classes of prefixes, directed edges describe the $\geq_{\mathcal{R}}$-accessibility relation, and every signed stick represents a main vein of the corresponding signed flower. In this graph representation, the $\mathcal{R}$-classes of prefixes are called nodes, the main vein associated with a node $n$ is denoted by $V(n)$, and the length of $V(n)$ by $l(V(n))$. 


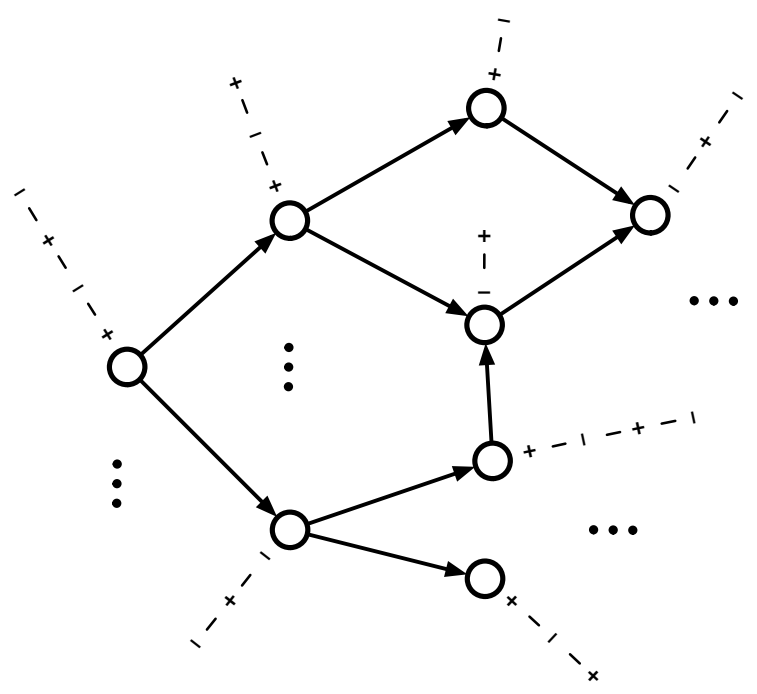

Figure 11. The pruned signed DAG representation of a finite pointed $\omega$-semigroup: a labeled DAG, where each node is associated with a signed integer describing the sign and the length of its corresponding main veins.

\section{MAIN ALGORITHM}

We now present the main algorithm that computes the $\mathbb{S} \mathbb{G}$-degree of every finite pointed $\omega$-semigroup. This algorithm works on the pruned signed DAG representation of finite pointed $\omega$-semigroups. It associates every finite pointed $\omega$-semigroup $(S, X)$ with a signed ordinal $\left[\varepsilon_{X}\right] \xi_{X}$. We will further prove that $d_{s g}(X)=\xi_{X}$, and that $X$ is self-dual if and only if $\varepsilon_{X}= \pm$, and $X$ is non-self dual if and only if $\varepsilon_{X} \in\{+,-\}$. This algorithm is a reformulation in terms of ordinals of Wagner's naming procedure $[16,21,23]$. We refer to Section 1.1 for basic definitions and facts about ordinals, ordinal arithmetic, and signed ordinals.

\section{Algorithm 4.1.}

INPUT a finite pointed $\omega$-semigroup $(S, X)$.

OUTPUT a signed ordinal $\left[\varepsilon_{X}\right] \xi_{X}$.

(1) Compute the pruned signed DAG representation of $(S, X)$.

(2) Define the function $n \longmapsto\left[\delta_{n}\right] \theta_{n}$ which associates to each node $n$ the signed ordinal $\left[\delta_{n}\right] \theta_{n}$ given by

$\delta_{n}=\left\{\begin{array}{ll}+ & \text { if the first element of } V(n) \text { is positive, } \\ - & \text { if the first element of } V(n) \text { is negative, }\end{array}\right.$ and $\theta_{n}=\omega^{l(V(n))}$ 
(3) Then, by backward induction, define the other function $n \longmapsto\left[\varepsilon_{n}\right] \xi_{n}$ which associates to each node $n$ the signed ordinal $\left[\varepsilon_{n}\right] \xi_{n}$ as follows.

(i) If $n$ is a sink, then $\left[\varepsilon_{n}\right] \xi_{n}=\left[\delta_{n}\right] \theta_{n}$, where $\left[\delta_{n}\right] \theta_{n}$ is the signed ordinal associated with $n$ by procedure (2).

(ii) If $n$ is not a sink, and $m_{1}, \ldots, m_{k}$ are all the direct successors of $n$ already associated with their respective signed ordinals $\left[\varepsilon_{1}\right] \xi_{1}, \ldots$, $\left[\varepsilon_{k}\right] \xi_{k}$ :

- If among $\left[\varepsilon_{1}\right] \xi_{1}, \ldots,\left[\varepsilon_{k}\right] \xi_{k}$, there is only one maximal signed ordinal $\left[\varepsilon_{m_{j}}\right] \xi_{m_{j}}$, then consider the Cantor Normal Form of base $\omega$ of the ordinal $\xi_{m_{j}}: \xi_{m_{j}}=\omega^{\alpha_{l}} \cdot \beta_{l}+\ldots+\omega^{\alpha_{0}} \cdot \beta_{0}$,

- If $\theta_{n}<\omega^{\alpha_{0}}$ or if both $\theta_{n}=\omega^{\alpha_{0}}$ and $\delta_{n}=\varepsilon_{m_{j}}$ (same signs), then set $\left[\varepsilon_{n}\right] \xi_{n}=\left[\varepsilon_{m_{j}}\right] \xi_{m_{j}}$.

- If $\theta_{n}>\omega^{\alpha_{0}}$ or if both $\theta_{n}=\omega^{\alpha_{0}}$ and $\delta_{n} \neq \varepsilon_{m_{j}}$ (opposite signs), then set $\left[\varepsilon_{n}\right] \xi_{n}=\left[\delta_{n}\right]\left(\xi_{m_{j}}+\theta_{n}\right)$.

- If among $\left[\varepsilon_{1}\right] \xi_{1}, \ldots,\left[\varepsilon_{k}\right] \xi_{k}$, there are two opposite maximal ordinals $\left[\varepsilon_{m_{i}}\right] \xi_{m_{i}}$ and $\left[\varepsilon_{m_{j}}\right] \xi_{m_{j}}$ (i.e. $\xi_{m_{i}}=\xi_{m_{j}}$ and $\varepsilon_{m_{i}} \neq \varepsilon_{m_{j}}$ ), then set $\left[\varepsilon_{n}\right] \xi_{n}=\left[\delta_{n}\right]\left(\xi_{m_{i}}+\theta_{n}\right)$.

(4) Finally, the finite pointed $\omega$-semigroup $(S, X)$ is associated with the signed ordinal $\left[\varepsilon_{X}\right] \xi_{X}$ as follows: let $\left[\varepsilon_{1}\right] \xi_{1}, \ldots,\left[\varepsilon_{p}\right] \xi_{p}$ be the signed ordinals associated by procedure (3) with all the respective sources $s_{1}, \ldots, s_{p}$ :

- If among $\left[\varepsilon_{1}\right] \xi_{1}, \ldots,\left[\varepsilon_{p}\right] \xi_{p}$, there is only one maximal signed ordinal $\left[\varepsilon_{\max }\right] \xi_{\max }$, then $\left[\varepsilon_{X}\right] \xi_{X}=\left[\varepsilon_{\max }\right] \xi_{\max }$.

- On the other hand, if among $\left[\varepsilon_{1}\right] \xi_{1}, \ldots,\left[\varepsilon_{p}\right] \xi_{p}$, there are two opposite maximal ordinals $[+] \xi_{\max }$ and $[-] \xi_{\max }$, then $\left[\varepsilon_{X}\right] \xi_{X}=[ \pm] \xi_{\max }$.

The following examples give several applications of this algorithm.

Example 4.2. Figure 12 illustrates the computation of Algorithm 4.1 on the DAG representation of a finite pointed semigroup $(S, X)$. In the top figure, every node $n$ is associated with its signed ordinal $\left[\delta_{n}\right] \theta_{n}$ given by procedure $(2)$. In the bottom figure, every node $n$ is associated with the two signed ordinals $\left[\delta_{n}\right] \theta_{n}$ (top) and $\left[\varepsilon_{n}\right] \xi_{n}$ (bottom) respectively given by procedures (2) and (3). The final signed ordinal associated with $(S, X)$ is is the second signed ordinal associated with the unique root, namely $[+]\left(\omega^{9}+\omega^{4} \cdot 2\right)$.

Example 4.3. Figure 13 illustrates another computation of Algorithm 4.1 on the DAG representation of a finite pointed semigroup $(T, Y)$. The final signed ordinal associated with $Y$ is the second signed ordinal associated with the two roots, namely $[ \pm]\left(\omega^{9}+\omega^{4} \cdot 2\right)$.

Next theorem states that Algorithm 4.1 computes the precise $\mathbb{S G}$-degree of any $\omega$-subset. The whole following section is devoted to proving this result.

Theorem 4.4. Let $(S, X)$ be a finite pointed $\omega$-semigroup, and let $\left[\varepsilon_{X}\right] \xi_{X}$ be the signed ordinal associated with $X$ by the main algorithm. Then $d_{s g}(X)=\xi_{X}$, and $X$ is self-dual if and only if $\left[\varepsilon_{X}\right]= \pm$. 

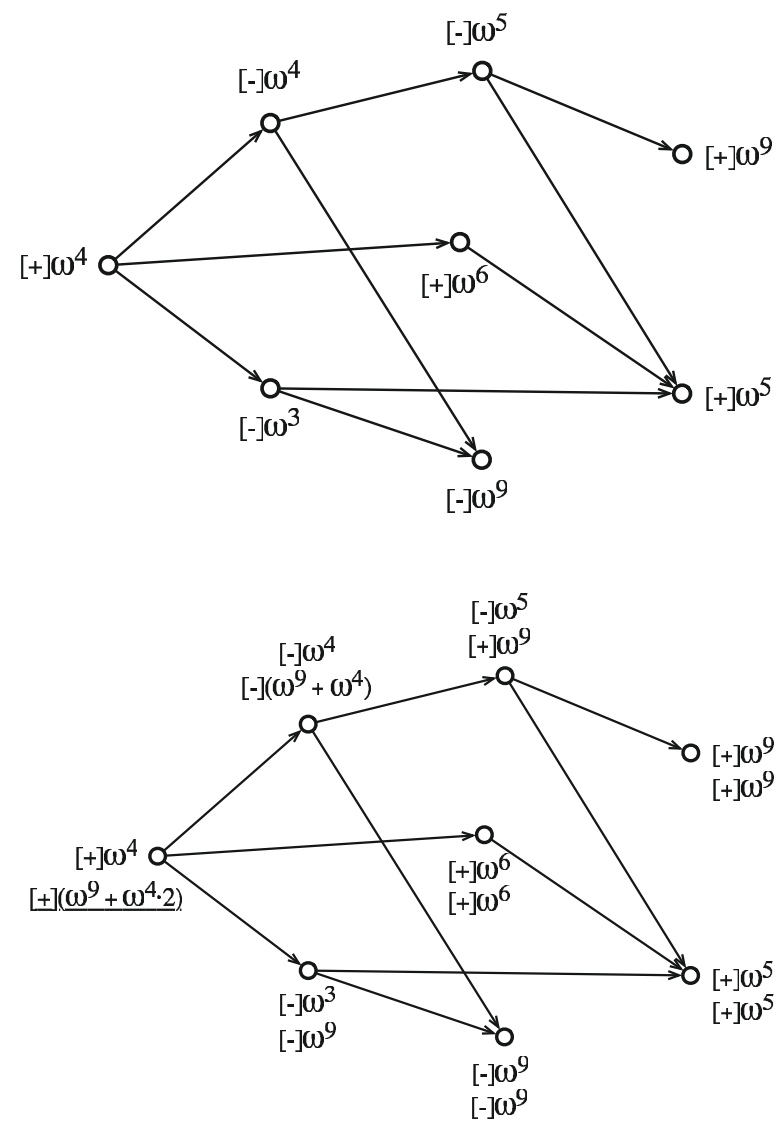

FigURE 12. Example of a computation of Algorithm 4.1.

Example 4.5. Let $A=\{a, b\}$, and let $K=\left(A^{*} a\right)^{\omega}$. The finite pointed $\omega$-semigroup $(S, X)$ given in Example 3.3 is the syntactic pointed $\omega$-semigroup of $K$. The computation of Algorithm 4.1 on $(S, X)$ gives $\left[\varepsilon_{X}\right] \xi_{X}=[-] \omega$, as illustrated in Figure 14 below. Therefore, $X$ is non-self-dual and $d_{s g}(X)=\omega$. The $\omega$-language $K$ is thence also non-self-dual with Wagner degree equal to $\omega$.

Example 4.6. Let $B=\{a, b, c\}$ and let $L=\left(a\{b, c\}^{*} \cup\{b\}\right)^{\omega}$ be an $\omega$-language over $B$. The finite pointed $\omega$-semigroup $(T, Y)$ given in Example 3.4 is the syntactic pointed $\omega$-semigroup of $L$. The computation of Algorithm 4.1 on $(T, Y)$ gives $\left[\varepsilon_{Y}\right] \xi_{Y}=[+] \omega^{2}$, as illustrated in Figure 15. Therefore, $Y$ is non-self-dual and $d_{s g}(Y)=\omega^{2}$. Hence $L$ is also non-self-dual with Wagner degree precisely $\omega^{2}$. 

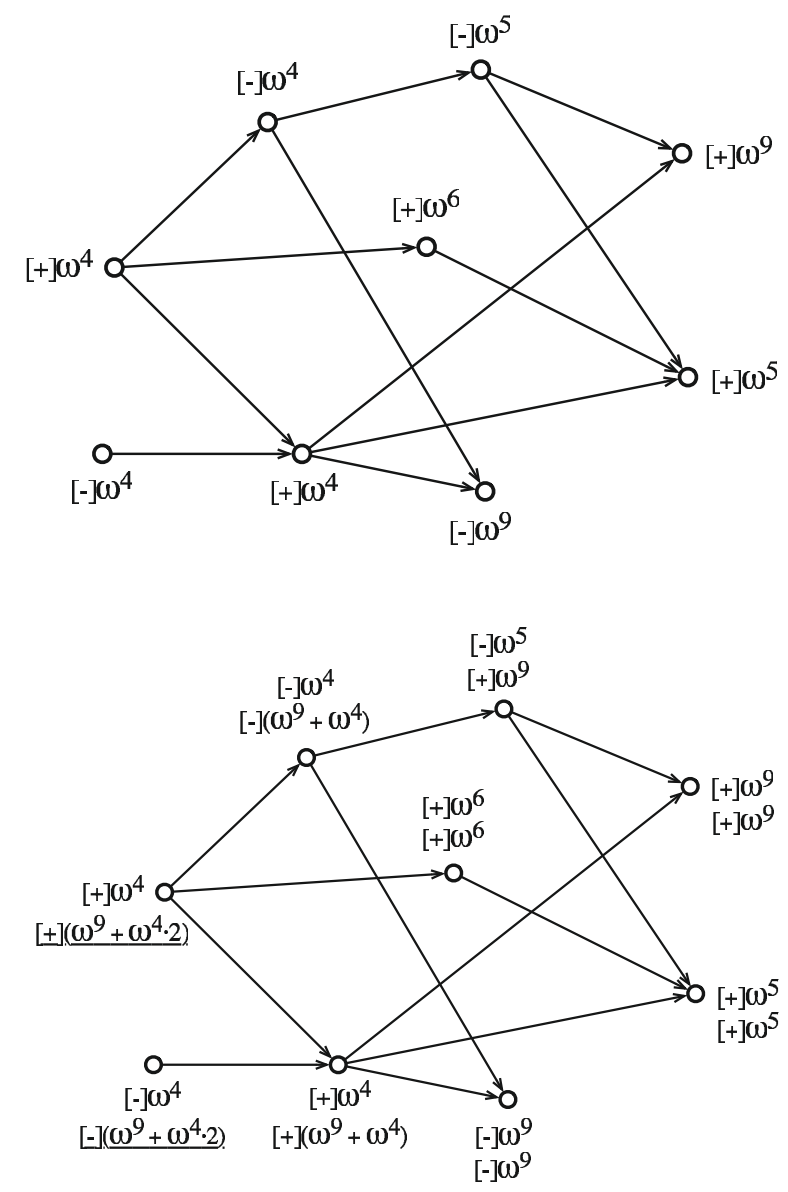

Figure 13. Another example of a computation of Algorithm 4.1.

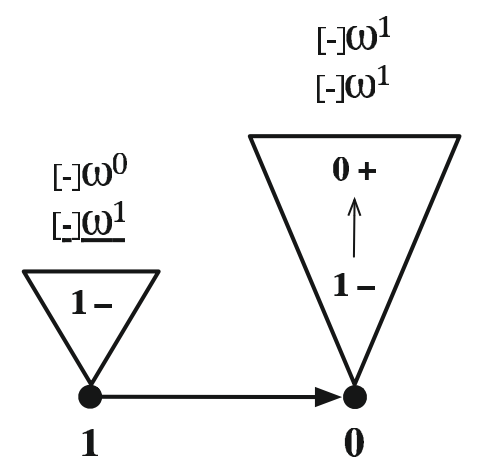

Figure 14. The signed DAG representation of $(S, X)$. 


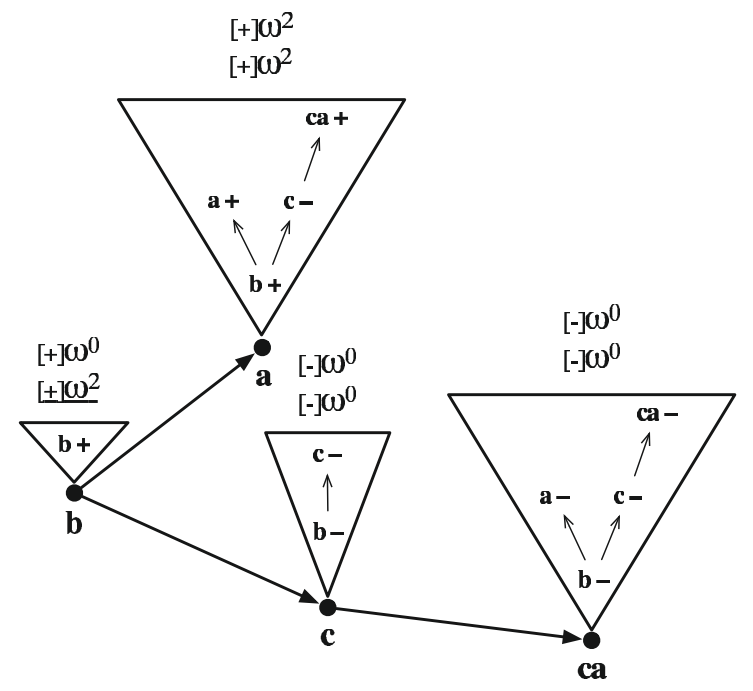

FigurE 15. The signed DAG representation of $(T, Y)$.

\section{CorReCtNess OF THE MAIN ALGORITHM}

This section is entirely devoted to proving Theorem 4.4. For this purpose, we introduce three infinite two-player games involving signed ordinals and finite pointed $\omega$-semigroups. The first one provides a game theoretical reformulation of the ordering on signed ordinals. The two other ones define two useful reductions on finite pointed $\omega$-semigroups and signed ordinals. From this point onward, every signed ordinal is assumed to be of the form $[\varepsilon] \xi$, with $\varepsilon \in\{+,-\}$ and $0<\xi<\omega^{\omega}$. Signed ordinals of the form $[ \pm] \xi$ will be considered separately at the end of the section.

The following preliminary results involve the notions of playground and cut defined in Section 1.1, as well as the notations of Algorithm 4.1. Hence, if $(S, X)$ is a finite pointed $\omega$-semigroup, then $\left[\varepsilon_{X}\right] \xi_{X}$ denotes the signed ordinal associated with $X$ after computation of Algorithm 4.1, and if $n$ is a node of the signed DAG representation of $(S, X)$, then $\left[\delta_{n}\right] \theta_{n}$ and $\left[\varepsilon_{n}\right] \xi_{n}$ are the signed ordinals associated with $n$ by procedures (2) and (3), respectively. The first results relates the playgrounds of $\left[\delta_{n}\right] \theta_{n}$ and $\left[\varepsilon_{n}\right] \xi_{n}$, and proves that the signed ordinals $\left[\varepsilon_{n}\right] \xi_{n}$ are decreasing along the $\geq_{\mathcal{R}}$-accessibility relation between the nodes.

Lemma 5.1. Let $(S, X)$ be a finite pointed $\omega$-semigroup, and let $n$ and $n^{\prime}$ be two nodes of the signed $D A G$ representation of $X$.

(1) Either $p g\left(\xi_{n}\right)>p g\left(\theta_{n}\right)$, or both $p g\left(\xi_{n}\right)=p g\left(\theta_{n}\right)$ and $\varepsilon_{n}=\delta_{n}$.

(2) If $n \geq \mathcal{R} n^{\prime}$, then $\left[\varepsilon_{n}\right] \xi_{n} \geq\left[\varepsilon_{n^{\prime}}\right] \xi_{n^{\prime}}$. 


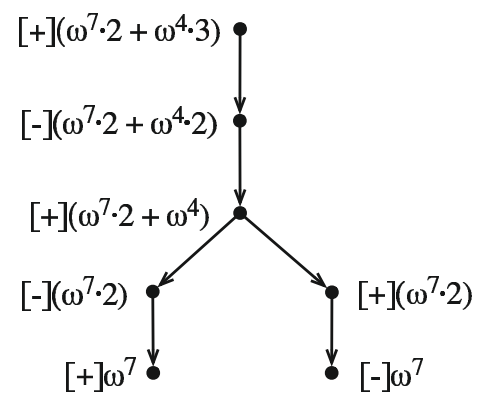

FIGURE 16. In the signed DAG representation of a finite pointed $\omega$-semigroup, for each node $n$, every cut of $\left[\varepsilon_{n}\right] \xi_{n}$ is accessible from $n$.

Proof. We use the notations of the main algorithm.

(1) We consider all possible values of $\left[\varepsilon_{n}\right] \xi_{n}$ computed by Algorithm 4.1. If $n$ is a sink, then $\left[\varepsilon_{n}\right] \xi_{n}=\left[\delta_{n}\right] \theta_{n}$. Thus obviously both $p g\left(\xi_{n}\right)=p g\left(\theta_{n}\right)$ and $\varepsilon_{n}=\delta_{n}$ hold. Otherwise, if $\left[\varepsilon_{n}\right] \xi_{n}=\left[\varepsilon_{m_{j}}\right] \xi_{m_{j}}$, then $p g\left(\xi_{n}\right)=\alpha_{0}$. Therefore, either $p g\left(\xi_{n}\right)>p g\left(\theta_{n}\right)$, or both $p g\left(\xi_{n}\right)=p g\left(\theta_{n}\right)$ and $\varepsilon_{n}=\delta_{n}$. Finally, if $\left[\varepsilon_{n}\right] \xi_{n}=\left[\delta_{n}\right]\left(\xi_{m_{j}}+\theta_{n}\right)$, then $p g\left(\xi_{n}\right)=p g\left(\theta_{n}\right)$, by definition of the ordinal sum.

(2) The signed ordinals $\left[\varepsilon_{n}\right] \xi_{n}$ are assigned recursively from the sinks to the sources of the signed DAG representation of $(S, X)$. In both cases, if $\left[\varepsilon_{n}\right] \xi_{n}=\left[\varepsilon_{m_{j}}\right] \xi_{m_{j}}$ or if $\left[\varepsilon_{n}\right] \xi_{n}=\left[\delta_{n}\right]\left(\xi_{m_{j}}+\theta_{n}\right)$, then $\left[\varepsilon_{n}\right] \xi_{n}$ is larger than the signed ordinals assigned to all its direct successors. Therefore, $\left[\varepsilon_{n}\right] \xi_{n}$ is larger than the signed ordinals assigned to all its successors.

Next result shows that, for every node $n$, all the cuts of $\left[\varepsilon_{n}\right] \xi_{n}$ are reachable from $n$. More precisely, for every node $n$ and every cut $c$ of $\left[\varepsilon_{n}\right] \xi_{n}$, there exists a node $n^{\prime}$ such that both $n>_{\mathcal{R}} n^{\prime}$ and $\left[\varepsilon_{n^{\prime}}\right] \xi_{n^{\prime}}=c$. This accessibility relation between cuts is illustrated in Figure 16. This property will be used to describe the strategy of an $\mathbb{S} \mathbb{G}$-player moving from cut to cut.

Lemma 5.2. Let $n$ be a node associated with the signed ordinal $\left[\varepsilon_{n}\right] \xi_{n}$, and let $[\varepsilon] \xi$ be a cut of $\left[\varepsilon_{n}\right] \xi_{n}$. Then there exists a node $n^{\prime}$ such that both $n>_{\mathcal{R}} n^{\prime}$ and $\left[\varepsilon_{n^{\prime}}\right] \xi_{n^{\prime}}=[\varepsilon] \xi$.

Proof. The proof goes by induction on $\xi_{n}$. If $\xi_{n}$ is of the form $\omega^{n_{k}}$, then there is no possible cut of $\left[\varepsilon_{n}\right] \xi_{n}$, thence nothing to prove in this case. Otherwise, two cases may occur.

(1) Assume that $\left[\varepsilon_{n}\right] \xi_{n}=\left[\varepsilon_{n}\right]\left(\omega^{n_{k}} \cdot p_{k}+\cdots+\omega^{n_{0}} \cdot\left(p_{0}+1\right)\right)$, for some $k \geq 0$ and $p_{0} \geq 0$. Procedure (3) of Algorithm 4.1 ensures that there exists a 
successor $n^{\prime}$ of $n\left(\right.$ possibly $\left.n^{\prime}=n\right)$ such that $\left[\varepsilon_{n^{\prime}}\right] \xi_{n^{\prime}}=\left[\varepsilon_{n}\right] \xi_{n},\left[\delta_{n^{\prime}}\right] \theta_{n^{\prime}}=$ $\left[\varepsilon_{n}\right] \omega^{n_{0}}$, and $\left[\varepsilon_{n^{\prime}}\right] \xi_{n^{\prime}}$ was updated as follows:

$$
\begin{aligned}
{\left[\varepsilon_{n^{\prime}}\right] \xi_{n^{\prime}} } & =\left[\delta_{n^{\prime}}\right]\left(\left(\omega^{n_{k}} \cdot p_{k}+\cdots+\omega^{n_{0}} \cdot p_{0}+\omega^{m_{l}} \cdot q_{l}+\cdots+\omega^{m_{0}} \cdot q_{0}\right)+\omega^{n_{0}}\right) \\
& =\left[\delta_{n^{\prime}}\right]\left(\omega^{n_{k}} \cdot p_{k}+\cdots+\omega^{n_{0}} \cdot\left(p_{0}+1\right)\right)=\left[\varepsilon_{n}\right] \xi_{n},
\end{aligned}
$$

for some $n_{0}>m_{l}>\ldots>m_{0} \geq 0$, or possibly $\omega^{m_{l}} \cdot q_{l}+\cdots+\omega^{m_{0}} \cdot q_{0}=0$. By definition of the main algorithm, there exists a successor $m$ of $n^{\prime}$ such that

$\left[\varepsilon_{m}\right] \xi_{m}=\left[\varepsilon_{m}\right]\left(\omega^{n_{k}} \cdot p_{k}+\cdots+\omega^{n_{0}} \cdot p_{0}+\omega^{m_{l}} \cdot q_{l}+\cdots+\omega^{m_{0}} \cdot q_{0}\right)$,

where $\varepsilon_{m}=+$ if and only if $\varepsilon_{n}=-$. By the induction hypothesis, since $\xi_{m}<\xi_{n}$, the node $m$ can access a node associated with each cut of $\left[\varepsilon_{m}\right] \xi_{m}$. Therefore, $m$ can also access a node associated with each cut of $\left[\varepsilon_{n}\right] \xi_{n}$, and so does $n$.

(2) Assume that $\left[\varepsilon_{n}\right] \xi_{n}=\left[\varepsilon_{n}\right]\left(\omega^{n_{k}} \cdot p_{k}+\cdots+\omega^{n_{1}} \cdot p_{1}+\omega^{n_{0}}\right)$, for some $k \geq 0$. The updating procedure (3) ensures that there exists a successor $n^{\prime}$ of $n$ (possibly $n^{\prime}=n$ ) such that $\left[\varepsilon_{n^{\prime}}\right] \xi_{n^{\prime}}=\left[\varepsilon_{n}\right] \xi_{n},\left[\delta_{n^{\prime}}\right] \theta_{n^{\prime}}=\left[\varepsilon_{n}\right] \omega^{n_{0}}$, and $\left[\varepsilon_{n^{\prime}}\right] \xi_{n^{\prime}}$ was updated as follows:

$$
\begin{aligned}
{\left[\varepsilon_{n^{\prime}}\right] \xi_{n^{\prime}} } & =\left[\delta_{n^{\prime}}\right]\left(\left(\omega^{n_{k}} \cdot p_{k}+\cdots+\omega^{n_{1}} \cdot p_{1}+\omega^{m_{l}} \cdot q_{l}+\cdots+\omega^{m_{0}} \cdot q_{0}\right)+\omega^{n_{0}}\right) \\
& =\left[\delta_{n^{\prime}}\right]\left(\omega^{n_{k}} \cdot p_{k}+\cdots+\omega^{n_{1}} \cdot p_{1}+\omega^{n_{0}}\right)=\left[\varepsilon_{n}\right] \xi_{n},
\end{aligned}
$$

for some $n_{1}>n_{0}>m_{l}>\ldots>m_{0} \geq 0$, or also possibly $\omega^{m_{l}} \cdot q_{l}+\cdots+$ $\omega^{m_{0}} \cdot q_{0}=0$.

- If $\omega^{m_{l}} \cdot q_{l}+\cdots+\omega^{m_{0}} \cdot q_{0} \neq 0$, the main algorithm ensures that there exists a successor $m$ of $n^{\prime}$ such that

$$
\left[\varepsilon_{m}\right] \xi_{m}=\left[\varepsilon_{m}\right]\left(\omega^{n_{k}} \cdot p_{k}+\cdots+\omega^{n_{1}} \cdot p_{1}+\omega^{m_{l}} \cdot q_{l}+\cdots+\omega^{m_{0}} \cdot q_{0}\right) .
$$

By the induction hypothesis, since $\xi_{m}<\xi_{n}$, the node $m$ can access a node associated with each cut of $\left[\varepsilon_{m}\right] \xi_{m}$. Therefore, $m$ can also access a node associated with each cut of $\left[\varepsilon_{n}\right] \xi_{n}$, and so does $n$.

- If $\omega^{m_{l}} \cdot q_{l}+\cdots+\omega^{m_{0}} \cdot q_{0}=0$, the main algorithm ensures that there exist two successors $m$ and $m^{\prime}$ of $n^{\prime}$ such that

$$
\begin{aligned}
{\left[\varepsilon_{m}\right] \xi_{m} } & =[+]\left(\omega^{n_{k}} \cdot p_{k}+\cdots+\omega^{n_{1}} \cdot p_{1}\right), \\
{\left[\varepsilon_{m^{\prime}}\right] \xi_{m^{\prime}} } & =[-]\left(\omega^{n_{k}} \cdot p_{k}+\cdots+\omega^{n_{1}} \cdot p_{1}\right) .
\end{aligned}
$$

By the induction hypothesis, since $\xi_{m}<\xi_{n}$, both nodes $m$ and $m^{\prime}$ can access a node associated with each cut of $\left[\varepsilon_{m}\right] \xi_{m}$. Finally, since $\left[\varepsilon_{m}\right] \xi_{m}$ and $\left[\varepsilon_{m^{\prime}}\right] \xi_{m^{\prime}}$ are the two largest cuts of $n$, and $n$ can access $m$ and $m^{\prime}$, then $n$ can access a node associated with each cut of $\left[\varepsilon_{n}\right] \xi_{n}$. 
We now introduce three infinite two-player games. The first one provides a game theoretical characterization of the ordering on signed ordinals. The two others involve finite pointed $\omega$-semigroups and signed ordinals.

Let $\left[\varepsilon_{I}\right] \xi_{I}$ and $\left[\varepsilon_{I I}\right] \xi_{I I}$ be two signed ordinals with $\varepsilon_{I}, \varepsilon_{I I} \in\{+,-\}$. The infinite two-player game $\mathbb{O}\left(\left[\varepsilon_{I}\right] \xi_{I},\left[\varepsilon_{I I}\right] \xi_{I I}\right)$ is defined as follows. First of all, Player I chooses a non-zero signed ordinal which is either $\left[\varepsilon_{I}\right] \xi_{I}$, or a cut of $\left[\varepsilon_{I}\right] \xi_{I}$, and Player II chooses a non-zero signed ordinal which is either $\left[\varepsilon_{I I}\right] \xi_{I I}$, or a cut of $\left[\varepsilon_{I I}\right] \xi_{I I}$. Then, the possible moves of players I and II are given as follows:

- Let $[\varepsilon] \xi$ be the last signed ordinal played by Player I. Then I can either choose a cut of $[\varepsilon] \xi$, or he can play a positive integer from his current playground: that is an integer $q$ such that $0 \leq q \leq p g(\xi)$.

- Similarly, let $[\delta] \eta$ be the last signed ordinal played by Player II. Then II can either choose a cut of $[\delta] \eta$, or play a positive integer from her current playground.

In other terms, each player decreases her/his signed ordinal cut by cut, and plays integers of his current playground in between. Player I begins. Player II is allowed to skip her turn, provided she plays infinitely often, whereas Player I is not allowed. At the end of the play, the infinite sequences respectively played by I and II consist of two finite strictly decreasing sequences of signed ordinals $\left[\varepsilon_{I, 0}\right] \xi_{I, 0}>$ $\ldots>\left[\varepsilon_{I, m}\right] \xi_{I, m}$ and $\left[\varepsilon_{I I, 0}\right] \xi_{I I, 0}>\ldots>\left[\varepsilon_{I I, n}\right] \xi_{I I, n}$, and two infinite sequences of integers. Let $i_{I}$ and $i_{I I}$ be the largest integers played infinitely often by I and II, respectively. We consider the following parity condition: Player I's play (resp. Player II's play) is said to be accepted if $\varepsilon_{I, m}=+\Leftrightarrow i_{I}$ is even (resp. $\varepsilon_{I I, n}=+\Leftrightarrow i_{I I}$ is even); it is called rejected otherwise. Then Player II wins $\mathbb{O}\left(\left[\varepsilon_{I}\right] \xi_{I},\left[\varepsilon_{I I}\right] \xi_{I I}\right)$ if and only if I and II's plays are either both accepted or both rejected. This game is illustrated in Figure 17. We now define via this game the following reduction on signed ordinals:

$$
\left[\varepsilon_{I}\right] \xi_{I} \leq_{O}\left[\varepsilon_{I I}\right] \xi_{I I} \text { iff Player II has a winning strategy in } \mathbb{O}\left(\left[\varepsilon_{I}\right] \xi_{I},\left[\varepsilon_{I I}\right] \xi_{I I}\right) \text {. }
$$

As usual, we set $\left[\varepsilon_{I}\right] \xi_{I}<_{O}\left[\varepsilon_{I I}\right] \xi_{I I}$ if and only if both $\left[\varepsilon_{I}\right] \xi_{I} \leq_{O}\left[\varepsilon_{I I}\right] \xi_{I I}$ and $\left[\varepsilon_{I I}\right] \xi_{I I} \leq_{O}\left[\varepsilon_{I}\right] \xi_{I}$ hold, and also $\left[\varepsilon_{I}\right] \xi_{I} \equiv_{O}\left[\varepsilon_{I I}\right] \xi_{I I}$ if and only if $\left[\varepsilon_{I}\right] \xi_{I} \leq_{O}$ $\left[\varepsilon_{I I}\right] \xi_{I I}$ and $\left[\varepsilon_{I I}\right] \xi_{I I} \leq_{O}\left[\varepsilon_{I}\right] \xi_{I}$.

Furthermore, the infinite two-player game $\mathbb{S} G \mathbb{O}(X,[\varepsilon] \xi)$ is defined as follows. Player I plays exactly the same way as in a game $\mathbb{S} G\left(X,{ }_{-}\right)$, and Player II plays as in a game $\mathbb{O}\left({ }_{-},[\varepsilon] \xi\right)$. Player II is allowed to skip her turn, but must play infinitely often, whereas Player I is not allowed to do so. Along the play, Player I builds an infinite sequence of elements $\left(s_{0}, s_{1}, \ldots\right)$, and Player II builds a finite sequence of signed ordinals $\left[\varepsilon_{I I, 0}\right] \xi_{I I, 0}>\ldots>\left[\varepsilon_{I I, n}\right] \xi_{I I, n}$, and an infinite sequence of integers. The winning condition is the following: Player II wins $\mathbb{S G O}(X,[\varepsilon] \xi)$ if and only if $\pi_{S}\left(s_{0}, s_{1}, \ldots\right) \in X \Leftrightarrow$ her play is accepted. Once again, this game induces the reduction

$X \leq_{O S G}[\varepsilon] \xi$ if and only if Player II has a winning strategy in $\mathbb{S G O}(X,[\varepsilon] \xi)$. 


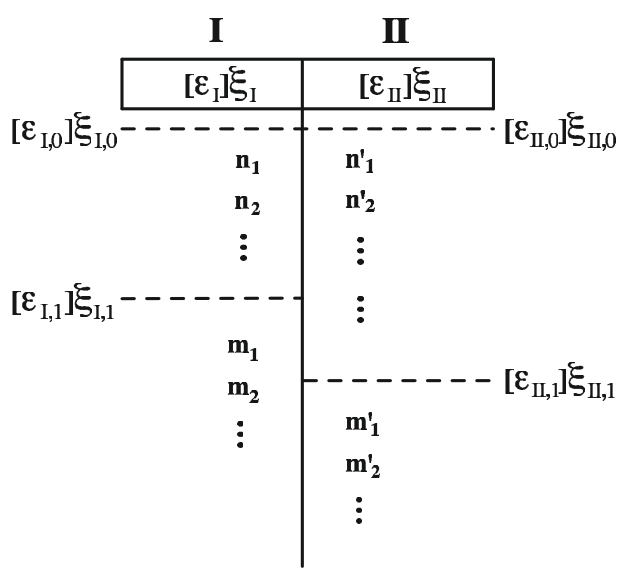

Figure 17 . The infinite game $\mathbb{O}\left(\left[\varepsilon_{I}\right] \xi_{I},\left[\varepsilon_{I I}\right] \xi_{I I}\right)$. II first choose the respective signed ordinals $\left[\varepsilon_{I, 0}\right] \xi_{I, 0}$ and $\left[\varepsilon_{I I, 0}\right] \xi_{I I, 0}$, and then play either integers from their current playgrounds, or some cut of their previous signed ordinal.

Finally, the infinite two-player game $\mathbb{O S G}([\varepsilon] \xi, X)$ is defined in a similar way. I plays exactly as in $\mathbb{O}\left([\varepsilon] \xi,{ }_{-}\right)$, and II plays as in $\left.\mathbb{S} \mathbb{G}_{-}, X\right)$. Player I begins and cannot skip his turn. Player II is allowed to skip her turn, provided she plays infinitely often. Along the play, Player I builds a finite sequence of signed ordinals $\left[\varepsilon_{I, 0}\right] \xi_{I, 0}>\ldots>\left[\varepsilon_{I, n}\right] \xi_{I, n}$, and an infinite sequence of integers, and Player II builds an infinite sequence $\left(s_{0}, s_{1}, \ldots\right)$ of elements of the semigroup involved. The winning condition is: Player II wins $\mathbb{O S} G([\varepsilon] \xi, X)$ if and only if Player I's play is accepted $\Leftrightarrow \pi_{S}\left(s_{0}, s_{1}, \ldots\right) \in X$. One more time, we define the corresponding reduction relation by

$[\varepsilon] \xi \leq_{O S G} X$ if and only if Player II has a winning strategy in $\mathbb{O S G}([\varepsilon] \xi, X)$.

We prove that the determinacy of these three specific games follows from Borel Wadge determinacy.

Proposition 5.3. For every signed ordinals $[\varepsilon] \xi$ and $\left[\varepsilon^{\prime}\right] \xi^{\prime}$, and every Borel $\omega$ subset $X$, the games $\mathbb{O}\left([\varepsilon] \xi,\left[\varepsilon^{\prime}\right] \xi^{\prime}\right), \mathbb{S} \mathbb{G} \mathbb{O}(X,[\varepsilon] \xi)$, and $\mathbb{O} \mathbb{S} \mathbb{G}([\varepsilon] \xi, X)$ are determined.

Proof. We reduce each of these games to an equivalent Wadge game with Borel winning condition. We conclude by Borel determinacy of Wadge games. More precisely, according to the rules of the $\mathbb{O}$-game, we let $L$ be the set of infinite words of the form

$$
\left(\left[\varepsilon_{0}\right] \xi_{0}\right) u_{0}\left(\left[\varepsilon_{1}\right] \xi_{1}\right) u_{1} \cdots\left(\left[\varepsilon_{n}\right] \xi_{n}\right) \alpha_{n}
$$

where $\left(\left[\varepsilon_{1}\right] \xi_{1}, \ldots,\left[\varepsilon_{n}\right] \xi_{n}\right)$ is a strictly descending sequence of signed ordinals such that each $\left[\varepsilon_{i+1}\right] \xi_{i+1}$ is a cut of $\left[\varepsilon_{i}\right] \xi_{i}$, each $u_{i}$ is a finite sequence of integers bounded by $p g\left(\xi_{i}\right)$, and $\alpha_{n}$ is an infinite sequence of integers bounded by $p g\left(\xi_{n}\right)$. 
We then equip $L$ with the usual topology over infinite words. Now, for every signed ordinal $[\varepsilon] \xi$, we let $L_{[\varepsilon] \xi} \subseteq L$ be the set of infinite words of the form $([\varepsilon] \xi) u_{0}\left(\left[\varepsilon_{1}\right] \xi_{1}\right) u_{1} \ldots\left(\left[\varepsilon_{k}\right] \xi_{k}\right) \alpha_{k}$ such that the largest integer appearing infinitely often in $\alpha_{k}$ is even if and only if $\left[\varepsilon_{k}\right]=+$. Then $L_{[\varepsilon] \xi}$ can be written as the conjunction of an open condition $\left(\boldsymbol{\Sigma}_{\mathbf{1}}^{\mathbf{0}}\right)$ and a parity condition $\left(B C\left(\boldsymbol{\Sigma}_{\mathbf{2}}^{\mathbf{0}}\right)\right)$, hence it is Borel. In addition, a given player has a winning strategy in $\mathbb{O}([\varepsilon] \xi,[\delta] \eta)$ if and only if this same player has a winning strategy in $\mathbb{W}\left(L_{[\varepsilon] \xi}, L_{[\delta] \eta}\right)$. Therefore, Borel Wadge determinacy implies the determinacy of $\mathbb{O}$-games. Similarly, a given player has a winning strategy in the game $\mathbb{S} G \mathbb{O}(X,[\varepsilon] \xi)$ if and only if the same player has a winning strategy in $\mathbb{W}\left(\pi_{S}^{-1}(X), L_{[\varepsilon] \xi}\right)$, where $\pi_{S}$ is the infinite product of the $\omega$-semigroup involved. Once again, Borel Wadge determinacy proves that $\mathbb{S G O}$-games are determined. The last case is proved in a symmetric way.

Example 5.4. Let $(S, X)$ be the finite pointed $\omega$-semigroup defined in Example 3.3. We show that $[-] \omega \leq_{O S G} X$ and $X \leq_{S G O}[-] \omega$. We will further prove that these two relations imply $d_{s g}(X)=\omega$. We first describe a winning strategy for Player II in $\mathbb{O S G}([-] \omega, X)$. On his first move, Player I is forced to choose the signed ordinal $[-] \omega$, thence Players I and II are forced to play elements 0 or 1 . When Player I plays 1, Player II plays 0, and when Player I plays 0, Player II plays 1 . Therefore, if I plays infinitely many 1 's, then II plays infinitely many 0 's, thus both plays are accepted. If I plays finitely many 1's, thus infinitely many 0 's, then II plays finitely many 0's, thus infinitely many 1's, and hence both plays are rejected. Therefore, II wins the game, thus $[-] \omega \leq_{O S G} X$. The very same strategy is winning for Player II in the game $\mathbb{S} \mathbb{G O}(X,[-] \omega)$, which shows that $X \leq_{S G O}[-] \omega$.

Example 5.5. Let $(T, Y)$ be the finite pointed $\omega$-semigroup defined in Example 3.4. Then one has $[+] \omega^{2} \leq_{O S G} Y$ and $Y \leq_{S G O}[+] \omega^{2}$ both hold. We will further prove that these two relations imply $d_{s g}(Y)=\omega^{2}$.

We now present the technical results involved in the proof of Theorem 4.4. First, we show that the $\mathbb{O}$-reduction and the classical ordering on signed ordinals coincide. Second, given a finite pointed $\omega$-semigroup $(S, X)$, the forthcoming Lemmas 5.8, 5.9 , and 5.10 prove that both relations $X \leq_{S G O}\left[\varepsilon_{X}\right] \xi_{X}$ and $\left[\varepsilon_{X}\right] \xi_{X} \leq_{O S G} X$ hold.

Lemma 5.6. Let $[\varepsilon] \xi$ and $\left[\varepsilon^{\prime}\right] \xi^{\prime}$ be two signed ordinals. Then $[\varepsilon] \xi \leq_{O}\left[\varepsilon^{\prime}\right] \xi^{\prime}$ if and only if $[\varepsilon] \xi \leq\left[\varepsilon^{\prime}\right] \xi^{\prime}$ (where $\leq$ is the natural ordering on signed ordinals defined in Section 1.1).

Proof.

$(\Leftarrow)$ Assume that $[\varepsilon] \xi \leq\left[\varepsilon^{\prime}\right] \xi^{\prime}$. We prove that Player II has a winning strategy in $\mathbb{O}\left([\varepsilon] \xi,\left[\varepsilon^{\prime}\right] \xi^{\prime}\right)$. II is in charge of a larger signed ordinal than I in the game $\mathbb{O}\left([\varepsilon] \xi,\left[\varepsilon^{\prime}\right] \xi^{\prime}\right)$. Therefore, along the play, she can choose her successive signed ordinals in order that her current playground is always larger than I's. More precisely, if I lately chose $\left[\varepsilon_{I}\right] \xi_{I}$, then she can always choose a signed ordinal $\left[\varepsilon_{I I}\right] \xi_{I I}$ such that either $p g\left(\xi_{I I}\right)>p g\left(\xi_{I}\right)$, or both $p g\left(\xi_{I I}\right)=$ $p g\left(\xi_{I}\right)$ and $\varepsilon_{I I}=\varepsilon_{I}$. In both cases, she can suitably answer to I's integers 
in order to produce a play of the same acceptance. She wins the game, thus $[\varepsilon] \xi \leq_{O}\left[\varepsilon^{\prime}\right] \xi^{\prime}$.

$(\Rightarrow)$ Assume that $[\varepsilon] \xi \not \leq\left[\varepsilon^{\prime}\right] \xi^{\prime}$. We prove that Player I has a winning strategy in $\mathbb{O}\left([\varepsilon] \xi,\left[\varepsilon^{\prime}\right] \xi^{\prime}\right)$. First of all, every time II skips her turn, I answers by playing 0 , which does not influence the acceptance of his current play. In addition, if II lately chose the signed ordinal $\left[\varepsilon_{I I}\right] \xi_{I I}$, then I can always choose a signed ordinal $\left[\varepsilon_{I}\right] \xi_{I}$ such that either $p g\left(\xi_{I}\right)>p g\left(\xi_{I I}\right)$, or both $p g\left(\xi_{I}\right)=p g\left(\xi_{I I}\right)$ and $\varepsilon_{I I} \neq \varepsilon_{I}$. In both cases, he can suitably answer to II's integers in order to produce a play of the opposite acceptance. He wins the game, thus $[\varepsilon] \xi \mathbb{L}_{O}\left[\varepsilon^{\prime}\right] \xi^{\prime}$.

Remark 5.7. In particular, given $0<\xi<\omega^{\omega}$, Player I has two winning strategies in the respective games $\mathbb{O}([+] \xi,[-] \xi)$ and $\mathbb{O}([-] \xi,[+] \xi)$. He always chooses a signed ordinal of the opposite sign as II's current one, copies every integer played by II, and plays 0 when II skips her turn. Therefore $[+] \xi \not_{O}[-] \xi$ and $[-] \xi \not_{O}[+] \xi$.

Lemma 5.8. Let $(S, X)$ be a finite pointed $\omega$ semigroup, let $n$ be a node of $X$, and let $X_{n}=\left\{x \in X \mid x=s e^{\omega}\right.$ for some $\left.s \in n\right\}$. Then

(1) $X_{n} \leq_{S G O}\left[\delta_{n}\right] \theta_{n}$;

(2) $\left[\delta_{n}\right] \theta_{n} \leq_{O S G} X_{n}$.

Proof. Let $V(n)$ be a main vein associated with $n$, and $s$ be the prefix such that $V(n) \subseteq \operatorname{petal}_{X}(s)$.

(1) We describe a winning strategy for Player II in the game $\mathbb{S G O}\left(X_{n},\left[\delta_{n}\right] \theta_{n}\right)$. As long as I's successive positions never reaches $n$, then II builds a rejecting play and wins. Otherwise, by Proposition 3.20, we may assume, without loss of generality, that I first plays the element $s$, and then restricts himself to playing only elements of $V(n)$. Hence, II chooses the signed ordinal $\left[\delta_{n}\right] \theta_{n}$ on her first move. Afterwards, for every idempotent $e$ played by I, she answers by playing the rank of $e$ in $V(n)$. The definition of $\left[\delta_{n}\right] \theta_{n}$ ensures that her current playground is large enough to do so. Moreover, again by definition of $\left[\delta_{n}\right] \theta_{n}$, I's play belongs to $X_{n}$ if and only if II's play is accepted. Therefore, Player II wins the game, hence $X_{n} \leq_{S G O}\left[\delta_{n}\right] \theta_{n}$.

(2) We describe a winning strategy for player II in $\mathbb{O S G}\left(\left[\delta_{n}\right] \theta_{n}, X_{n}\right)$. Since $\theta_{n}$ is of the form $\omega^{k}$, it has no cut, hence I is forced to choose the signed ordinal $\left[\delta_{n}\right] \theta_{n}$ on his first move. Then II plays the prefix $s$ on her first move. Afterwards, for each integer $0 \leq n \leq p g\left(\theta_{n}\right)$ played by I, she answers by the idempotent of $V(n)$ whose rank is precisely $n$. By definition of $\left[\delta_{n}\right] \theta_{n}$, I's play is accepted if and only if II's play belongs to $X_{n}$. Consequently, Player II wins the game, thus $\left[\delta_{n}\right] \theta_{n} \leq_{O S G} X_{n}$.

Lemma 5.9. Let $(S, X)$ be a finite pointed $\omega$-semigroup associated with the signed ordinal $\left[\varepsilon_{X}\right] \xi_{X}$, with $\varepsilon_{X} \in\{+,-\}$. Then $X \leq_{S G O}\left[\varepsilon_{X}\right] \xi_{X}$.

Proof. We show that Player II has a winning strategy in $\mathbb{S G O}\left(X,\left[\varepsilon_{X}\right] \xi_{X}\right)$. By Proposition 3.24, we may assume that I restricts himself to only playing elements of $V(n)$ while his successive positions remain in a given node $n$. Hence, II first 
chooses the signed ordinal $\left[\varepsilon_{X}\right] \xi_{X}$, and then plays as follows. Every time I's play reaches a node $n$, two cases may occur.

(1) The signed ordinal $\left[\varepsilon_{n}\right] \xi_{n}$ is a cut of $\left[\varepsilon_{X}\right] \xi_{X}$. Then Player II chooses the signed ordinal $\left[\varepsilon_{n}\right] \xi_{n}$, and plays her integers as described in Lemma 5.8 (1). Lemma 5.1 (1) guarantees that her current playground is large enough to play this way.

(2) The signed ordinal $\left[\varepsilon_{n}\right] \xi_{n}$ is not a cut of $\left[\varepsilon_{X}\right] \xi_{X}$. Then $\left[\varepsilon_{n}\right] \xi_{n}$ can be written as $\left[\varepsilon_{n}\right](\alpha+\beta)$, where $\left[\varepsilon_{n}\right] \alpha$ is the largest cut of $\left[\varepsilon_{X}\right] \xi_{X}$ strictly below $\left[\varepsilon_{n}\right] \xi_{n}$. Hence, II chooses the signed ordinal $\left[\varepsilon_{n}\right] \alpha$. By Lemma $5.1(2)$, since the signed ordinals associated to each node are decreasing along the accessibility relation, the ordinal $\left[\varepsilon_{n}\right] \alpha$ is indeed smaller than or equal to the previous ordinal chosen by II. In addition, this choice ensures that II's playground is larger than $p g\left(\xi_{n}\right)$. Thence, player II can play her integers as described in Lemma $5.8(1)$.

By finiteness and acyclicity of the signed DAG representation of $(S, X)$, I's play will eventually become confined to a certain node $n^{\prime \prime}$ after a finite amount of time. Then, II plays according to the corresponding signed ordinal, as described in cases (1) or (2). In both cases, Lemma 5.8 (1) ensures that she wins the game. Therefore, $X \leq_{S G O}\left[\varepsilon_{X}\right] \xi_{X}$.

Lemma 5.10. Let $(S, X)$ be a finite pointed $\omega$-semigroup associated with the signed ordinal $\left[\varepsilon_{X}\right] \xi_{X}$, with $\varepsilon_{X} \in\{+,-\}$. Then $\left[\varepsilon_{X}\right] \xi_{X} \leq_{O S G} X$.

Proof. We describe a winning strategy for Player II in $\mathbb{O S G}\left(\left[\varepsilon_{X}\right] \xi_{X}, X\right)$. Every time I chooses a signed ordinal $[\varepsilon] \xi$, II reaches one of the accessible $\leq_{\mathcal{R}}$-largest node $n$ such that $\left[\varepsilon_{n}\right] \xi_{n}=[\varepsilon] \xi$. Lemma 5.2 ensures the existence of such a node. When I plays some integer, II answers exactly as described in Lemma 5.8 (2). By finiteness of strictly descending sequences of signed ordinals, I is forced to choose a final cut of $\left[\varepsilon_{X}\right] \xi_{X}$. Then, II reaches the suitable corresponding node, and plays as described in Lemma 5.8 (2). She thus wins the game, proving that $\left[\varepsilon_{X}\right] \xi_{X} \leq_{O S G} X$.

The forthcoming Proposition 5.11 shows that the $\leq_{O}$-relation on signed ordinals coincides with the $\leq_{S G}$-relation on $\omega$-subsets. Moreover, we prove that an $\omega$-subset $X$ is self-dual if and only if $\varepsilon_{X}= \pm$. We also show that any self-dual $\omega$-subsets and any non-self-dual one which is located just one level below it in the $\mathbb{S} G$-hierarchy both share the same ordinal by Algorithm 4.1. The full proof of Theorem 4.4 follows from these statements.

Proposition 5.11. Let $(S, X)$ and $(T, Y)$ be two finite pointed $\omega$-semigroups associated with the respective signed ordinals $\left[\varepsilon_{X}\right] \xi_{X}$ and $\left[\varepsilon_{Y}\right] \xi_{Y}$, and such that $\varepsilon_{X}, \varepsilon_{X} \in\{+,-\}$. Then $X \leq_{S G} Y$ if and only if $\left[\varepsilon_{X}\right] \xi_{X} \leq\left[\varepsilon_{Y}\right] \xi_{Y}$.

Proof. If $X \leq_{S G} Y$, then Lemmas 5.9 and 5.10 show that $\left[\varepsilon_{X}\right] \xi_{X} \leq_{O S G} X \leq_{S G}$ $Y \leq_{S G O}\left[\varepsilon_{Y}\right] \xi_{Y}$. By composition of strategies, one obtains $\left[\varepsilon_{X}\right] \xi_{X} \leq_{O}\left[\varepsilon_{Y}\right] \xi_{Y}$. Therefore, Lemma 5.6 implies $\left[\varepsilon_{X}\right] \xi_{X} \leq\left[\varepsilon_{Y}\right] \xi_{Y}$. Conversely, if $\left[\varepsilon_{X}\right] \xi_{X} \leq\left[\varepsilon_{Y}\right] \xi_{Y}$, then Lemma 5.6 shows that $\left[\varepsilon_{X}\right] \xi_{X} \leq_{O}\left[\varepsilon_{Y}\right] \xi_{Y}$. Hence, Lemmas 5.9 and 5.10 imply 
$X \leq_{S G O}\left[\varepsilon_{X}\right] \xi_{X} \leq_{O}\left[\varepsilon_{Y}\right] \xi_{Y} \leq_{O S G} Y$. By composition of strategies, it follows that $X \leq_{S G} Y$.

Proposition 5.12. Let $(S, X)$ be a finite pointed $\omega$-semigroup, and let $\left[\varepsilon_{X}\right] \xi_{X}$ be the signed ordinal associated with $X$ by the main algorithm.

(1) $X$ is non-self-dual if and only if $\varepsilon_{X} \in\{+,-\}$,

(2) $X$ is self-dual if and only if $\varepsilon_{X}= \pm$.

Proof. We prove that if $\varepsilon_{X} \in\{+,-\}$ then $X$ is non-self-dual, and if $\varepsilon_{X}= \pm$, then $X$ is self-dual. The two converse directions follow from contrapositives of these statements.

(1) If $\varepsilon_{X} \in\{+,-\}$, Procedure (4) of Algorithm 4.1 shows that there exists a source $\bar{s}$ of the signed DAG representation of $(S, X)$, such that $\left[\varepsilon_{\bar{s}}\right] \xi_{\bar{s}}=\left[\varepsilon_{X}\right] \xi_{X}$. Now, let $s$ be a prefix of the $\mathcal{R}$-class $\bar{s}$, and consider the set $s^{-1} X$. The main algorithm applied on $\left(S, s^{-1} X\right)$ shows that $\left[\varepsilon_{s^{-1} X}\right] \xi_{s^{-1} X}=\left[\varepsilon_{\bar{s}}\right] \xi_{\bar{s}}=\left[\varepsilon_{X}\right] \xi_{X}$. Therefore, Proposition 5.11 shows that $s^{-1} X \equiv_{S G} X$. By Proposition 2.3, the set $X$ is non-self-dual.

(2) If $\left[\varepsilon_{X}\right] \xi_{X}=[ \pm] \xi_{X}$, Procedure (4) shows that there exist two sources $\bar{s}$ and $\bar{t}$ of the signed DAG representation of $(S, X)$, such that $\left[\varepsilon_{\bar{s}}\right] \xi_{\bar{s}}=[+] \xi_{X}$ and $\left[\varepsilon_{\bar{t}}\right] \xi_{\bar{t}}=[-] \xi_{X}$. Since the signed DAG representations of $(S, X)$ and $\left(S, X^{c}\right)$ have opposite signs, there also exist two sources $\bar{s}^{\prime}$ and $\bar{t}^{\prime}$ of the signed DAG representation of $\left(S, X^{c}\right)$ such that $\left[\varepsilon_{\bar{s}^{\prime}}\right] \xi_{\bar{s}^{\prime}}=[+] \xi_{X}$ and $\left[\varepsilon_{\bar{t}^{\prime}}\right] \xi_{\bar{t}^{\prime}}=[-] \xi_{X}$. Now, let $s \in \bar{s}, t \in \bar{t}, s^{\prime} \in \bar{s}^{\prime}$, and $t^{\prime} \in \bar{t}^{\prime}$, and consider the sets $s^{-1} X$, $t^{-1} X, s^{-1} X^{c}$, and $t^{\prime-1} X^{c}$. One has

$$
\begin{aligned}
{\left[\varepsilon_{s^{-1} X}\right] \xi_{s^{-1} X} } & =\left[\varepsilon_{\bar{s}}\right] \xi_{\bar{s}}=[+] \xi_{X}=\left[\varepsilon_{\bar{s}^{\prime}}\right] \xi_{\bar{s}^{\prime}}=\left[\varepsilon_{s^{\prime-1} X^{c}}\right] \xi_{s^{\prime-1} X^{c}} \\
{\left[\varepsilon_{t^{-1} X}\right] \xi_{t^{-1} X} } & =\left[\varepsilon_{\bar{t}}\right] \xi_{\bar{t}}=[-] \xi_{X}=\left[\varepsilon_{\bar{t}^{\prime}}\right] \xi_{\bar{t}^{\prime}}=\left[\varepsilon_{t^{\prime-1} X^{c}}\right] \xi_{t^{\prime-1} X^{c}}
\end{aligned}
$$

We now prove that Player II has a winning strategy in $\mathbb{S} G\left(X, X^{c}\right)$. Since $S$ is finite, after finitely many moves, I is forced to reach a prefix position $u$ belonging to some $\mathcal{R}$-class of prefixes $\bar{u}$. Hence, he becomes in charge of the set $u^{-1} X$. The maximality properties of $\bar{s}$ and $\bar{t}$ ensure that either

$$
\begin{aligned}
{\left[\varepsilon_{u^{-1} X}\right] \xi_{u^{-1} X} } & =\left[\varepsilon_{\bar{u}}\right] \xi_{\bar{u}} \leq\left[\varepsilon_{\bar{s}}\right] \xi_{\bar{s}}=\left[\varepsilon_{s^{\prime-1} X^{c}}\right] \xi_{s^{\prime-1} X^{c}} \text { or } \\
{\left[\varepsilon_{u^{-1} X}\right] \xi_{u^{-1} X} } & =\left[\varepsilon_{\bar{u}}\right] \xi_{\bar{u}} \leq\left[\varepsilon_{\bar{t}}\right] \xi_{\bar{t}}=\left[\varepsilon_{t^{\prime-1} X^{c}}\right] \xi_{t^{\prime-1} X^{c}},
\end{aligned}
$$

thus Proposition 5.11 shows that either $u^{-1} X \leq_{S G} s^{-1} X^{c}$, or $u^{-1} X \leq_{S G}$ $t^{\prime-1} X^{c}$. Thence, for every $u \in P\left(S_{+}\right)$, there exists $v \in\left\{s^{\prime}, t^{\prime}\right\}$ such that II has a winning strategy $\sigma_{u}$ in $\mathbb{S} \mathbb{G}\left(u^{-1} X, v^{-1} X^{c}\right)$. Therefore, II first skips her turn until I reaches a prefix position $u$, then plays the required $v$, and finally applies the corresponding strategy $\sigma_{u}$. She wins the game $\mathbb{S} \mathbb{G}\left(X, X^{c}\right)$. Therefore, $X \leq_{S G} X^{c}$, and $X$ is self-dual.

Proposition 5.13. Let $(S, X)$ and $(T, Y)$ be two finite pointed $\omega$-semigroups such that $\left[\varepsilon_{X}\right] \xi_{X}=[+] \xi$ and $\left[\varepsilon_{Y}\right] \xi_{Y}=[ \pm] \xi$, for some $0<\xi<\omega^{\omega}$. Then $X<_{S G} Y$, and there is no pointed $\omega$-semigroup $(U, Z)$ satisfying $X<_{S G} Z<_{S G} Y$. 
Proof. We first prove that $X<_{S G} Y$. Since $\left[\varepsilon_{Y}\right] \xi_{Y}=[ \pm] \xi$, there exist two sources $\bar{s}$ and $\bar{t}$ of the signed DAG representation of $(T, Y)$ such that $\left[\varepsilon_{\bar{s}}\right] \xi_{\bar{s}}=[+] \xi$ and $\left[\varepsilon_{\bar{t}}\right] \xi_{\bar{t}}=[-] \xi$. Now, let $s \in \bar{s}$ and $t \in \bar{t}$, and consider the sets $s^{-1} Y$ and $t^{-1} Y$. One has

$$
\begin{aligned}
{\left[\varepsilon_{s^{-1} Y}\right] \xi_{s^{-1} Y} } & =\left[\varepsilon_{\bar{s}}\right] \xi_{\bar{s}}=[+] \xi=\left[\varepsilon_{X}\right] \xi_{X}, \\
{\left[\varepsilon_{t^{-1} Y}\right] \xi_{t^{-1} Y} } & =\left[\varepsilon_{\bar{t}}\right] \xi_{\bar{t}}=[-] \xi=\left[\varepsilon_{X^{c}}\right] \xi_{X^{c}}
\end{aligned}
$$

thus Proposition 5.11 shows that $s^{-1} Y \equiv_{S G} X$ and $t^{-1} Y \equiv_{S G} X^{c}$. In particular, $X \leq_{S G} s^{-1} Y \leq_{S G} Y$, hence $X \leq_{S G} Y$. Moreover, Proposition 5.12 shows that $X$ is non-self-dual and $Y$ is self-dual. Therefore $X<_{S G} Y$. We now prove the second part of the proposition. Let $Z>_{S G} X$. Then $X \equiv_{S G} s^{-1} Y<_{S G} Z$, and also $X^{c} \equiv_{S G} t^{-1} Y<_{S G} Z$. We prove that $Y \leq_{S G} Z$, by describing a winning strategy for Player II in $\mathbb{S G}(Y, Z)$. Since $T$ is finite, after finitely many moves, I is forced to reach a prefix position $u$ belonging to some $\mathcal{R}$-class of prefixes $\bar{u}$. Then, he finds himself in charge of the set $u^{-1} Y$. The maximality properties of $\bar{s}$ and $\bar{t}$ ensure that either

$$
\begin{aligned}
& {\left[\varepsilon_{u^{-1} Y}\right] \xi_{u^{-1} Y}=\left[\varepsilon_{\bar{u}}\right] \xi_{\bar{u}} \leq\left[\varepsilon_{\bar{s}}\right] \xi_{\bar{s}}=\left[\varepsilon_{s^{-1} Y}\right] \xi_{s^{-1} Y} \text { or }} \\
& {\left[\varepsilon_{u^{-1} Y}\right] \xi_{u^{-1} Y}=\left[\varepsilon_{\bar{u}}\right] \xi_{\bar{u}} \leq\left[\varepsilon_{\bar{t}}\right] \xi_{\bar{t}}=\left[\varepsilon_{t^{-1} Y}\right] \xi_{t^{-1} Y},}
\end{aligned}
$$

and thus Proposition 5.11 shows that either $u^{-1} Y \leq_{S G} s^{-1} Y<_{S G} Z$, or $u^{-1} Y \leq_{S G}$ $t^{-1} Y<_{S G} Z$. Hence, for every $u \in P\left(U_{+}\right)$, II has a winning strategy $\sigma_{u}$ in the game $\mathbb{S G}\left(u^{-1} Y, Z\right)$. Therefore, II skips her turn until I reaches such a position $u$, and then applies $\sigma_{u}$. She wins $\mathbb{S} \mathbb{G}(Y, Z)$, therefore $Y \leq_{S G} Z$.

Theorem 5.14. Let $(S, X)$ be finite pointed $\omega$-semigroup, and let $\left[\varepsilon_{X}\right] \xi_{X}$ be the signed ordinal associated with $X$ by Algorithm 4.1. Then $d_{s g}(X)=\xi_{X}$.

Proof. First, consider the mapping which associates every non-self-dual $\omega$-subset $X$ with its corresponding signed ordinal $\left[\varepsilon_{X}\right] \xi_{X}$ (with $\varepsilon_{X} \in\{+,-\}$ ). Propositions 5.12 (1) and 5.11 prove that this mapping is an embedding from the $\mathbb{F} \mathbb{S} G$-hierarchy of non-self-dual $\omega$-subsets into the hierarchy of signed ordinals of the form $[+] \xi$ or $[-] \xi$. The following section carries the proof that this mapping is onto. Therefore, $d_{s g}(X)=\xi_{X}$ holds for every non-self-dual $\omega$-subset $X$. In addition, Propositions 5.12 and 5.13 prove that self-dual $\omega$-subsets and the non-self-dual ones located right below in the $\mathbb{F} \mathbb{S G}$-hierarchy are associated with the same ordinal by the main algorithm. Therefore, $d_{s g}(X)=\xi_{X}$ holds for every self-dual $\omega$-subset $X$.

\section{Building pointed $\omega$-SEMigroups of ANy Given SG-DEGREe}

This section describes the algebraic counterpart of some ordinal operations. Given two finite pointed $\omega$-semigroups $(S, X)$ and $(T, Y)$, and any integer $n$, we successively describe the finite pointed $\omega$-semigroups $(S \oplus T, X \oplus Y),(S \odot n, X \odot n)$, 
and $(S \odot \omega, X \odot \omega)$ such that

$$
\begin{aligned}
d_{s g}(X \oplus Y) & =d_{s g}(X)+d_{s g}(Y), \\
d_{s g}(X \odot n) & =d_{s g}(X) \cdot n, \\
d_{s g}(X \odot \omega) & =d_{s g}(X) \cdot \omega .
\end{aligned}
$$

Consequently, starting from either the empty or the full $\omega$-subset of $\mathbb{S} \mathbb{G}$-degree 1 , one may build by induction an $\omega$-subset of any given $\mathbb{S} \mathbb{G}$-degree (strictly between 0 and $\left.\omega^{\omega}\right)$.

Let $S=\left(\left(S_{+}, *\right), S_{\omega}\right)$ and $T=\left(\left(T_{+}, \circledast\right), T_{\omega}\right)$ be two finite $\omega$-semigroups, and let $X \subseteq S_{\omega}$ and $Y \subseteq T_{\omega}$ be two non-self-dual $\omega$-subsets. Let also $\left(S_{+}^{\prime}, *^{\prime}\right)$ be a disjoint copy of the semigroup $\left(S_{+}, *\right)$ (i.e. $a^{\prime} \in S_{+}^{\prime}$ if and only if $a \in S_{+}$, and $a^{\prime} *^{\prime} b^{\prime}=c^{\prime}$ if and only if $\left.a * b=c\right)$. We consider the set

$$
(S \oplus T)_{+}=T_{+}^{1} \cup S_{+}^{1} \cup S_{+}^{\prime 1} \cup\{0\}
$$

equipped with the following operation

$$
a \cdot b=\left\{\begin{array}{cl}
0 & \text { if } a=0 \text { or } b=0, \\
0 & \text { if } a \in S_{+}^{1} \text { and } b \in S_{+}^{\prime 1}, \\
0 & \text { if } a \in S_{+}^{\prime 1} \text { and } b \in S_{+}^{1}, \\
a & \text { if } a \in S_{+}^{1} \text { and } b \in T_{+}^{1}, \\
b & \text { if } b \in S_{+}^{1} \text { and } a \in T_{+}^{1}, \\
a & \text { if } a \in S_{+}^{\prime 1} \text { and } b \in T_{+}^{1}, \\
b & \text { if } b \in S_{+}^{\prime 1} \text { and } a \in T_{+}^{1}, \\
a \circledast b & \text { if } a \text { and } b \text { belong to } T_{+}^{1}, \\
a * b & \text { if } a \text { and } b \text { belong to } S_{+}^{1}, \\
a *^{\prime} b & \text { if } a \text { and } b \text { belong to } S_{+}^{\prime 1} .
\end{array}\right.
$$

The element 0 is a zero; the product of any element of $S_{+}^{1}$ with any element of $S_{+}^{\prime} 1$ is 0 , and vice versa; the product of any two elements of either $T_{+}^{1}$, or $S_{+}^{1}$, or $S_{+}^{\prime}$, coincides with the products of the respective monoids $\left(T_{+}^{1}, \circledast\right),\left(S_{+}^{1}, *\right)$ or $\left(S_{+}^{\prime 1}, *\right)$; elements of $S_{+}^{1}$ and $S_{+}^{\prime 1}$ absorb the elements of $T_{+}^{1}$ from the left and the right, as illustrated by the following tabular:

\begin{tabular}{c|cccc}
$\cdot \nearrow$ & $T_{+}^{1}$ & $S_{+}^{1}$ & $S_{+}^{\prime 1}$ & 0 \\
\hline$T_{+}^{1}$ & table of $T_{+}^{1}$ & absorption by $S_{+}^{1}$ & absorption by $S_{+}^{\prime 1}$ & 0 \\
$S_{+}^{1}$ & absorption by $S_{+}^{1}$ & table of $S_{+}^{1}$ & 0 & 0 \\
$S_{+}^{\prime 1}$ & absorption by $S_{+}^{\prime 1}$ & 0 & table of $S_{+}^{\prime 1}$ & 0 \\
0 & 0 & 0 & 0 & 0
\end{tabular}

Lemma 6.1. The structure $\left((S \oplus T)_{+}, \cdot\right)$ is a semigroup. 


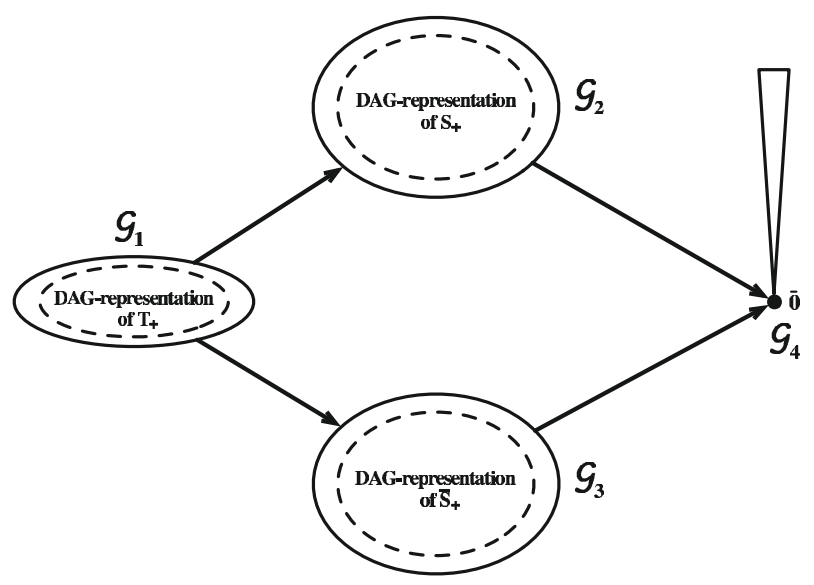

Figure 18. The DAG representation of $(S \oplus T)_{+}$. The accessibility relation between two DAGs $\mathcal{G}_{i}$ and $\mathcal{G}_{j}$ means that each node of $\mathcal{G}_{j}$ is $\geq_{\mathcal{R}}$-accessible from each node of $\mathcal{G}_{i}$.

Proof. The respective operations of $S_{+}^{1}, S_{+}^{\prime} 1$, and $T_{+}^{1}$, and the absorption relations from the left and from the right are associative. Adding a zero does not affect the associativity. Therefore, the operation defined on $(S \oplus T)_{+}$is associative.

The DAG representation of $(S \oplus T)_{+}$, consists of four sub-DAGs $\mathcal{G}_{1}, \mathcal{G}_{2}, \mathcal{G}_{3}$, and $\mathcal{G}_{4}$, induced by the respective elements of $T_{+}^{1}, S_{+}^{1}, S_{+}^{\prime 1}$, and by 0 . These DAGs satisfy the following properties:

- $\mathcal{G}_{1}$ is the DAG representation of $T_{+}^{1}$, therefore it contains the DAG representation of $T_{+}$.

- $\mathcal{G}_{2}$ is the DAG representation of $S_{+}^{1}$, possibly enriched by some new linked pairs induced by the products of the form $x \cdot y$, for $x \in S_{+}^{1}$ and $y \in T_{+}^{1}$. Hence, it contains the DAG representation of $S_{+}$.

- Similarly, $\mathcal{G}_{3}$ contains the DAG representation of $S_{+}^{\prime}$.

- $\mathcal{G}_{4}$ is the single-petal flower flower $(\overline{0})$ associated with the $\mathcal{R}$-class of prefixes $\overline{0}=\{0\}$. This petal contains all idempotents of $(S \oplus T)_{+}$.

These DAGs are related as follows: $\mathcal{G}_{1}$ is not $\geq_{\mathcal{R}}$-accessible from any other $\mathcal{G}_{i} ; \mathcal{G}_{2}$ and $\mathcal{G}_{3}$ are both $\geq_{\mathcal{R}}$-accessible from $\mathcal{G}_{1}$, but there is no $\geq_{\mathcal{R}}$-accessibility relation between them; $\mathcal{G}_{4}$ is $\geq_{\mathcal{R}}$-accessible from $\mathcal{G}_{1}, \mathcal{G}_{2}$, and $\mathcal{G}_{3}$. The DAG representation of $(S \oplus T)+$ is illustrated in Figure 18 .

The finite semigroup $(S \oplus T)_{+}$can be naturally extended to the finite $\omega$-semigroup

$$
S \oplus T=\left((S \oplus T)_{+},(S \oplus T)_{\omega}\right),
$$

where $(S \oplus T)_{\omega}=\left\{[s, e] \mid(s, e)\right.$ is a linked pair of $\left.(S \oplus T)_{+}\right\}$. Moreover, since the DAG representation of $T_{+}$is contained in $\mathcal{G}_{1}$, there exists a signature of $\mathcal{G}_{1}$ 
corresponding to an $\omega$-subset $\bar{Y} \subseteq(S \oplus T)_{\omega}$, such that $d_{s g}(\bar{Y})=d_{s g}(Y)$ and $\bar{Y} \equiv_{S G} Y$. Since the DAG representation of $S_{+}$is contained in $\mathcal{G}_{2}$, there also exists a signature of $\mathcal{G}_{2}$ corresponding to an $\omega$-subset $\bar{X} \subseteq(S \oplus T)_{\omega}$, such that $d_{s g}(\bar{X})=d_{s g}(X)$, and $\bar{X} \equiv_{S G} X$. By the same argument again, there exists a signature of $\mathcal{G}_{3}$ corresponding to an $\omega$-subset $\bar{X}^{\prime} \subseteq(S \oplus T)_{\omega}$, such that $d_{s g}\left(\bar{X}^{\prime}\right)=$ $d_{s g}(X)$, but $\bar{X}^{\prime} \equiv_{S G} X^{c}$. Using all these notations, one obtains the following result.

\section{Proposition 6.2.}

- If $d_{s g}(X)>1$ or $d_{s g}(Y)>1$, by setting $X \oplus Y=\bar{X} \cup \bar{X}^{\prime} \cup \bar{Y} \subseteq(S \oplus T)_{\omega}$, one has $d_{s g}(X \oplus Y)=d_{s g}(X)+d_{s g}(Y)$.

- If $d_{s g}(X)=d_{s g}(Y)=1$, by setting $X \oplus Y=\left\{[0, e] \mid e \in E\left((S \oplus T)_{+}\right)\right\} \subseteq$ $(S \oplus T)_{\omega}$, one has $d_{s g}(X \oplus Y)=d_{s g}(X)+d_{s g}(Y)=2$.

Proof. For the first case, let $r_{1}, r_{2}, r_{3}$ be the respective roots of $\mathcal{G}_{1}, \mathcal{G}_{2}$, and $\mathcal{G}_{3}$. The main algorithm applied separately to the sub-DAGs $\mathcal{G}_{1}, \mathcal{G}_{2}, \mathcal{G}_{3}$ assigned according to $\bar{Y}, \bar{X}, \bar{X}^{\prime}$ respectively gives $\left[\varepsilon_{r_{1}}\right] \xi_{r_{1}}=[+] d_{s g}(Y),\left[\varepsilon_{r_{2}}\right] \xi_{r_{2}}=[+] d_{s g}(X)$, and $\left[\varepsilon_{r_{3}}\right] \xi_{r_{3}}=[-] d_{s g}(X)$. Then, the accessibility relations between these DAGs imply that $d_{s g}(X \oplus Y)=d_{s g}(X)+d_{s g}(Y)$. In the second case, the set flower $X(\overline{0})$ contains only positive idempotents, and every other signed flower contains only negative idempotents. Therefore, the main algorithm gives $d_{s g}(X \oplus Y)=\omega^{0} \cdot 2=2=$ $d_{s g}(X)+d_{s g}(Y)$.

We now describe the algebraic counterpart of the ordinal finite multiplication. Let $(S, X)$ be a finite pointed $\omega$-semigroup. For any integer $n>0$, we define the finite pointed $\omega$-semigroup $(S \odot n, X \odot n)$ by induction on $n$ as follows:

- $(S \odot 1, X \odot 1)=(S, X)$,

- $S \odot(n+1)=(S \odot n) \oplus S$, and $X \odot(n+1)=(X \odot n) \oplus X$.

Proposition 6.3. Let $n>0$, then $d_{s g}(X \odot n)=d_{s g}(X) \cdot n$.

Proof. A direct consequence of Proposition 6.2.

We finally focus on the algebraic counterpart of the ordinal multiplication by $\omega$. We recall that, given any ordinal $\xi$ with Cantor normal form $\xi=\omega^{n_{k}} \cdot p_{k}+$ $\cdots+\omega^{n_{0}} \cdot p_{0}$, the equality $\xi \cdot \omega=\omega^{n_{k}+1}$ holds.

Let $S=\left(S_{+}, S_{\omega}\right)$ be a finite $\omega$-semigroup, and $X \subseteq S_{\omega}$, such that $d_{s g}(X)=$ $\xi=\sum_{i=k}^{0} \omega^{n_{i}} \cdot p_{i}$. We then consider the finite monoid

$$
(S \odot \omega)_{+}=\left(S_{+} \cup\{1\}, \cdot\right)
$$

equipped with the operation of $S_{+}$completed as follows: $a \cdot 1=1 \cdot a=a$, for all $a \in(S \odot \omega)_{+}$. The DAG representation of $(S \odot \omega)_{+}$, illustrated in Figures 19 and 20 , corresponds to the following transformation of the DAG representation of $S_{+}$:

- The flower flower $(\overline{1})$ associated with the $\mathcal{R}$-class of prefixes $\overline{1}=\{1\}$ appears. It simply consists of the single-petal petal $(1)=\{1\}$. The $\mathcal{R}$-class $\overline{1}$ can $\geq_{\mathcal{R}}$-access any other $\mathcal{R}$-class of prefixes $\bar{s}$. 

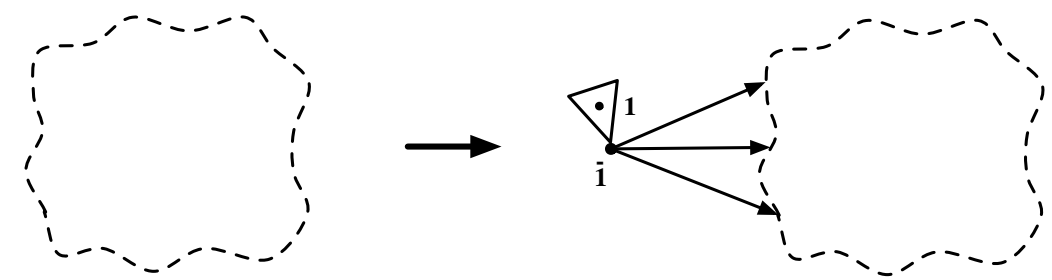

Figure 19. The transformation of the DAG representation of $S_{+}$ into the one of $(S \odot \omega)_{+}$: the new flower flower $(\overline{1})$ associated with the $\mathcal{R}$-class of prefixes $\overline{1}$ appears.

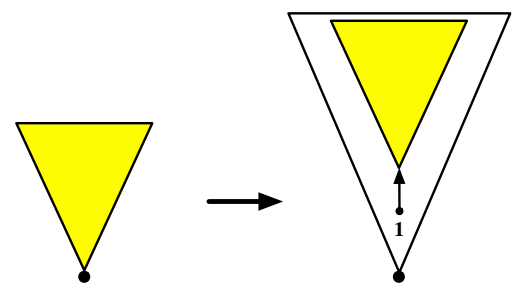

FIGURE 20. The transformation of a petal of $S_{+}$into a petal of $(S \odot \omega)_{+}$: the new idempotent 1 , strictly $\leq$-larger than any other, appears.

- The idempotent 1 , strictly $\leq$-larger than any other, appears in each petal of each flower of $S_{+}$. Therefore, the length of every chain of idempotents of $S_{+}$ is increased by 1 .

Moreover, since $d_{s g}(X)=\xi=\sum_{i=k}^{0} \omega^{n_{i}} \cdot p_{i}$, there exists at least one chain of idempotents $e_{0}>\cdots>e_{n_{k}}$ in some petal of the DAG representation of $S_{+}$. Consequently, one can find the chain of idempotents $1>e_{0}>\cdots>e_{n_{k}}$ in some petal of the DAG representation of $(S \odot \omega)_{+}$. Finally, the monoid $(S \odot \omega)_{+}$can be extended to the finite $\omega$-semigroup

$$
S \odot \omega=\left((S \odot \omega)_{+},(S \odot \omega)_{\omega}\right),
$$

where $\left.(S \odot \omega)_{\omega}\right)=\left\{[s, e] \mid(s, e)\right.$ is a linked pair of $\left.(S \odot \omega)_{+}\right\}$. Using all these notations, one obtains the following proposition.

Proposition 6.4. Let $s$ be a prefix of $(S \odot \omega)_{+}$such that the chain of idempotents $1>e_{0}>\ldots>e_{n_{k}}$ belongs to $\operatorname{petal}(s)$. Let us also set

$$
X \odot \omega=\left\{\left[s, e_{2 i}\right] \mid 0 \leq 2 i \leq n_{k}\right\} \subseteq(S \odot \omega)_{\omega},
$$

then $d_{s g}(X \odot \omega)=d_{s g}(X) \cdot \omega=\omega^{n_{k}+1}$.

Proof. The signature according to $X \odot \omega$ yields the unique maximal alternating chain $1>e_{0}>\ldots>e_{n_{k}}$ of length $n_{k}+1$ in the signed DAG representation 
of $(S \odot \omega, X \odot \omega)$. By Algorithm 4.1 and Theorem 5.14, one has $d_{s g}(X \odot \omega)=$ $\omega^{n_{k}+1}=d_{s g}(X) \cdot \omega$.

\section{NORMAL FORMS}

We now describe the algebraic invariants of the $\mathbb{F} \mathbb{S G}$-hierarchy. As in $[8,18,21]$, we prove that the $\mathbb{S} \mathbb{G}$-degree of $(S, X)$ is completely characterized by some kind of maximal alternating tree(s) contained in the signed DAG representation of $(S, X)$ - called the normal form of $(S, X)$. Then any two finite pointed $\omega$-semigroups share the same $\mathbb{S} G$-degree if and only if they have the same normal form, up to some relation of bisimilarity. The normal form of $(S, X)$ is a reformulation in this algebraic context of the notions of maximal $\xi$-chains presented in [8], or maximal $\mu_{\alpha}$-alternating tree described in [18], or also maximal binary tree-like sequences of superchains described in [21].

In the sequel, the signed DAG representation of finite pointed $\omega$-semigroups are regarded as labeled DAGs of the form $G=(V, E, p)$, where $p: V \longrightarrow\{+,-\} \times \mathbb{N}_{+}$ is a priority function which associates with every node $n$ the sign and length of the main vein $V(n)$.

We first introduce a notion of bisimulation over DAGs. Let $G=(V, E, p)$ and $G^{\prime}=\left(V^{\prime}, E^{\prime}, p^{\prime}\right)$ be two finite DAGs, where $p: V \longrightarrow\{+,-\} \times \mathbb{N}_{+}$and $p^{\prime}: V^{\prime} \longrightarrow\{+,-\} \times \mathbb{N}_{+}$are priority functions. A bisimulation over $G$ and $G^{\prime}$ is a left-and-right-total binary relation $B \subseteq V \times V^{\prime}$ such that $\left(n, n^{\prime}\right) \in B$ if and only if

- when $n$ and $n^{\prime}$ are sinks, then $p(n)=p^{\prime}\left(n^{\prime}\right)$;

- when $n$ or $n^{\prime}$ are not sinks, then $p(n)=p^{\prime}\left(n^{\prime}\right)$, and for every edge $(n, m) \in E$, there exists an edge $\left(n^{\prime}, m^{\prime}\right) \in E^{\prime}$ such that $\left(m, m^{\prime}\right) \in B$, and conversely, for every edge $\left(n^{\prime}, m^{\prime}\right) \in E^{\prime}$, there exists an edge $(n, m) \in E$ such that $\left(m, m^{\prime}\right) \in B$.

When there exists a bisimulation relation over $G$ and $G^{\prime}$, we say that $G$ and $G^{\prime}$ are bisimilar and write $G \approx G^{\prime}$. As a matter of fact, the DAGs $G$ and $G^{\prime}$ are bisimilar if and only if they contain the same kind of paths, i.e. for every path in $G$, there exists a path in $G^{\prime}$ visiting exactly the same priorities, and conversely, for every path in $G^{\prime}$, one can also find a path in $G$ visiting the same priorities.

The definition of bisimultation can be apprehended by means of games. To this end, we define the finite two-player game with perfect information $\mathbb{B} \mathbb{S}\left(G, G^{\prime}\right)$, where Player II tries to show that $G$ and $G^{\prime}$ are bisimilar, whereas Player I tries to show the opposite. The rules are the following:

- On his first move, I chooses a source of either $G$ or $G^{\prime}$. If he chooses a source $s$ of $G$, II must answer by choosing a source $s^{\prime}$ of $G^{\prime}$ such that $p(s)=p^{\prime}\left(s^{\prime}\right)$. If he chooses a source $s^{\prime}$ of $G^{\prime}$, II must answer by choosing a source $s$ of $G$ such that $p(s)=p^{\prime}\left(s^{\prime}\right)$.

- After every move of II, let $n \in V$ and $n^{\prime} \in V^{\prime}$ be the two nodes previously chosen respectively by I and II. Then, if it still exists, I chooses either a successor of $n$, or a successor of $n^{\prime}$. If he chooses a successor $m$ of $n$, then II 
must answer by choosing a successor $m^{\prime}$ of $n^{\prime}$ such that $p(m)=p^{\prime}\left(m^{\prime}\right)$. If he chooses a successor $m^{\prime}$ of $n^{\prime}$, then II must answer by choosing a successor $m$ of $n$ such that $p(m)=p^{\prime}\left(m^{\prime}\right)$.

If II is not able to answer correctly to I's move, she looses. If both players cannot choose a further successor node, II wins. Otherwise, the player which cannot choose a successor node whereas his opponent can do so looses the game.

Proposition 7.1. Let $G=(V, E, p)$ and $G^{\prime}=\left(V^{\prime}, E^{\prime}, p^{\prime}\right)$ be two finite DAGs. Then $G \approx G^{\prime}$ if and only if Player II has a winning strategy in $\mathbb{B I S}\left(G, G^{\prime}\right)$.

Proof. If $G \approx G^{\prime}$, there exists a bisimulation relation $B$ over $G$ and $G^{\prime}$ which induces the following winning strategy for Player II in $\mathbb{B I S}\left(G, G^{\prime}\right)$ : every time I chooses a node $x \in V$, II answers by an appropriate node $x^{\prime} \in V^{\prime}$ such that $\left(x, x^{\prime}\right) \in B$, and every time I chooses a node $x^{\prime} \in V^{\prime}$, II answers by a node $x \in V$ such that $\left(x, x^{\prime}\right) \in B$. Conversely, assume that Player II has a winning strategy in $\mathbb{B} \mathbb{I S}\left(G, G^{\prime}\right)$. Then for every path $\left(x_{0}, \ldots, x_{n}\right)$ in $G$, there exists a path $\left(x_{0}^{\prime}, \ldots, x_{n}^{\prime}\right)$ in $G^{\prime}$ such that $p\left(x_{i}\right)=p^{\prime}\left(x_{i}^{\prime}\right)$, for all $i$; and conversely, for every path $\left(y_{0}^{\prime}, \ldots, y_{n}^{\prime}\right)$ in $G^{\prime}$, there exists a path $\left(y_{0}, \ldots, y_{n}\right)$ in $G^{\prime}$, such that $p\left(y_{i}\right)=p^{\prime}\left(y_{i}^{\prime}\right)$, for all $i$. The set $B$ of such pairs $\left(x_{i}, x_{i}^{\prime}\right)$ and $\left(y_{i}, y_{i}^{\prime}\right)$ obtained by considering II's answer to every possible paths $\left(x_{0}, \ldots, x_{n}\right)$ in $G$ and $\left(y_{0}, \ldots, y_{n}\right)$ in $G^{\prime}$ is a bisimulation over $G$ and $G^{\prime}$. Therefore, $G \approx G^{\prime}$.

We now define the tree representation of any signed ordinals $[\varepsilon] \xi$ by induction on the Cantor normal form of $\xi$. This representation is inspired by the notion of a $\xi$-chain introduced by Duparc in [8].

(1) If $[\varepsilon] \xi$ is of the form $[+] \omega^{n} \cdot p$ (respectively $[-] \omega^{n} \cdot p$ ), for some integers $n \geq 0$ and $p>0$, its tree representation consists of a "linear" sequence of $p$ accessible nodes alternately labeled by $+n$ and $-n$ (respectively $-n$ and $+n)$, as illustrated in Figure 21 .

(2) If $[\varepsilon] \xi$ is of the form $[ \pm] \omega^{n} \cdot p$, for some integers $n \geq 0$ and $p>0$, its tree representation consists of the two disjoint tree representations of $[+] \omega^{n} \cdot p$ and $[-] \omega^{n} \cdot p$, as illustrated in Figure 21.

(3) If the Cantor normal form of $[\varepsilon] \xi$ is of the form $[+]\left(\eta+\omega^{n} \cdot p\right)$ (respectively $[-]\left(\eta+\omega^{n} \cdot p\right)$ ), for some $0<\eta<\omega^{\omega}$, and some integers $n \geq 0$ and $p>0$, its tree representation consists of the tree representation of $[+] \omega^{n} \cdot p$ (respectively $\left.[-] \omega^{n} \cdot p\right)$ related to the two disjoint tree representations of $[+] \eta$ and $[-] \eta$, as illustrated in Figure 22.

(4) If the Cantor normal form of $[\varepsilon] \xi$ is of the form $[ \pm]\left(\eta+\omega^{n} \cdot p\right)$, for some $0<\eta<\omega^{\omega}$, and some integers $n \geq 0$ and $p>0$, its tree representation consists of the two disjoint tree representations of $[+]\left(\eta+\omega^{n} \cdot p\right)$ and $[-]\left(\eta+\omega^{n} \cdot p\right)$.

Example 7.2. Figures 23 and 24 illustrate the tree representations of the respective signed ordinals $[-]\left(\omega^{5} \cdot 4+\omega^{3} \cdot 3+\omega^{2} \cdot 5\right)$ and $[ \pm]\left(\omega^{3} \cdot 3+\omega^{2} \cdot 5\right)$.

The tree representation of $[\varepsilon] \xi$ is an encoding of the Cantor normal form of $\xi$, with some additional property according to the sign $\varepsilon$. Hence, it is uniquely 


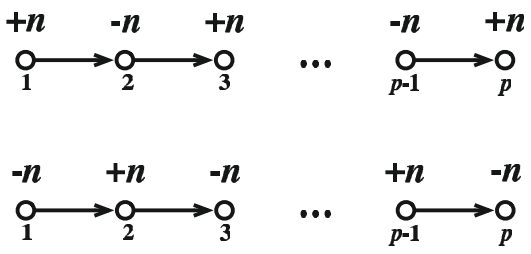

FIgURE 21. Tree representations of the signed ordinals $[+] \omega^{n} \cdot p$ and $[-] \omega^{n} \cdot p$. The union of these two graphs is the tree representation of $[ \pm] \omega^{n} \cdot p$. Every time there is an edge from $i$ to $j$, and from $j$ to $k$, there is also an edge from $i$ to $k$, but these transitive edges are not represented, for reasons of clarity.

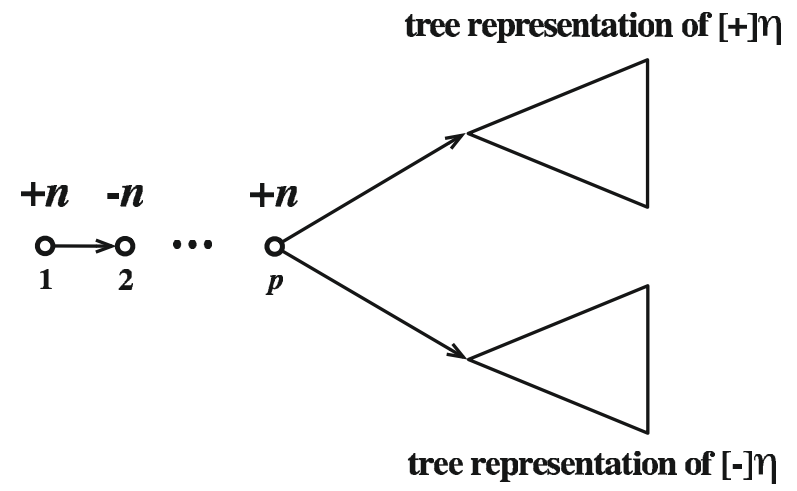

FIGURE 22. The tree representation of the signed ordinal $[+](\eta+$ $\left.\omega^{n} \cdot p\right)$. The tree representation of $[-]\left(\eta+\omega^{n} \cdot p\right)$ consists of the same DAG, but with an initial sequence of nodes with opposite signs.

determined, for each signed ordinal $[\varepsilon] \xi$. It has been defined in order to satisfy the following properties.

Lemma 7.3. When applied on the tree representation of $[\varepsilon] \xi$, the main algorithm outputs precisely $[\varepsilon] \xi$.

Proof. The proof goes by induction on the Cantor normal form of $[\varepsilon] \xi$. We prove the result for the case $\varepsilon \in\{+,-\}$. The case $\varepsilon= \pm$ is a direct consequence. If $[\varepsilon] \xi$ is of the form $[\varepsilon] \omega^{n} \cdot p$, for some $n \geq 0$ and $p>0$, the result is true. If the Cantor normal form of $[\varepsilon] \xi$ is of the form $[\varepsilon]\left(\eta+\omega^{n} \cdot p\right)$, its tree representation consists of the tree representation of $[\varepsilon] \omega^{n} \cdot p$ related to the two disjoint tree representations of $[+] \eta$ and $[-] \eta$. By the induction hypothesis, the two disjoint subtree representations of $[+] \eta$ and $[-] \eta$ are associated with the respective signed 


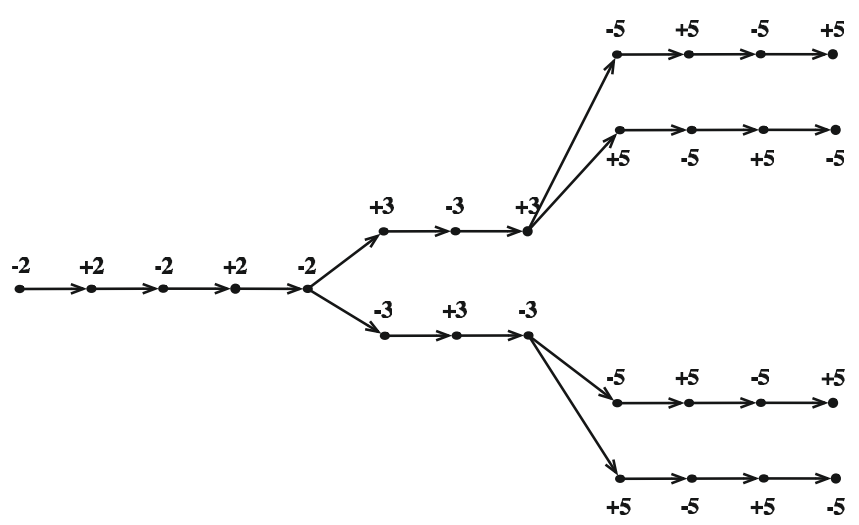

Figure 23. Tree representation $[-]\left(\omega^{5} \cdot 4+\omega^{3} \cdot 3+\omega^{2} \cdot 5\right)$.
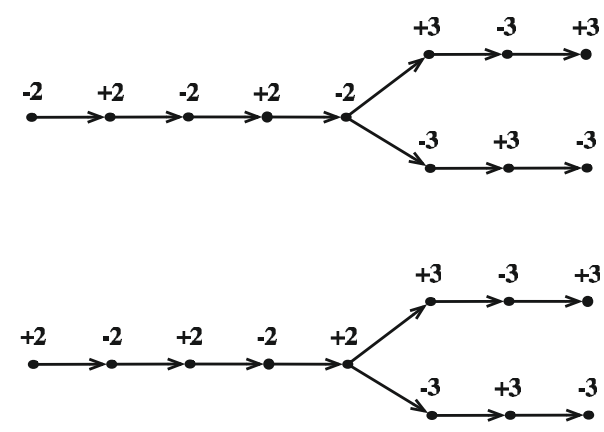

FiguRE 24. Tree representation of $[ \pm]\left(\omega^{3} \cdot 3+\omega^{2} \cdot 5\right)$.

ordinals $[+] \eta$ and $[-] \eta$. By definition of the Cantor normal form, $\omega^{n}$ is strictly below the every factor $\omega^{i}$ appearing in $\eta$. Therefore, the main algorithm associates the signed ordinal $[\varepsilon]\left(\eta+\omega^{n} \cdot p\right)=[\varepsilon] \xi$ with the root of the tree representation of $[\varepsilon] \xi$.

Lemma 7.4. The tree representations of $[\varepsilon] \xi$ and $\left[\varepsilon^{\prime}\right] \xi^{\prime}$ are bisimilar if and only if $[\varepsilon] \xi=\left[\varepsilon^{\prime}\right] \xi^{\prime}$.

Proof. Let $T$ and $T^{\prime}$ be the respective tree representations of $[\varepsilon] \xi$ and $\left[\varepsilon^{\prime}\right] \xi^{\prime}$. If $[\varepsilon] \xi=\left[\varepsilon^{\prime}\right] \xi^{\prime}$, then $T=T^{\prime}$, thus obviously $T \approx T^{\prime}$. Conversely, assume that $[\varepsilon] \xi \neq\left[\varepsilon^{\prime}\right] \xi^{\prime}$. Then two cases may occur. Firstly, if $\xi=\xi^{\prime}$ but $\varepsilon \neq \varepsilon^{\prime}$, then $T$ and $T$ are the very same trees, but with opposite priorities. Therefore, $T$ and $T^{\prime}$ do not contain the same paths, hence they are not bisimilar. Secondly, if $\xi>\xi^{\prime}$, then $T$ is a tree representation containing strictly more nodes than $T^{\prime}$, or strictly larger priorities then $T^{\prime}$. Hence, $T$ and $T^{\prime}$ do not contain the same paths, and they are not bisimilar. The case $\xi^{\prime}>\xi$ is symmetric. 
Given a finite pointed $\omega$-semigroup $(S, X)$, a normal form of $(S, X)$ is a subgraph $G$ of the signed DAG representation of $(S, X)$ containing a minimal number of nodes and edges, and such that an $\mathbb{S} G$-player restricting his moves inside $G$ is exactly as strong as if he were in charge of the whole DAG of $(S, X)$. We prove that the normal form of $(S, X)$ is precisely the tree representation of $\left[\varepsilon_{X}\right] d_{s g}(X)$ (up to bisimilarity), and hence it is unique, up to bisimilarity. Therefore, any two finite pointed $\omega$-semigroups have the same $\mathbb{S} G$-degree if and only if they have the same normal form.

Proposition 7.5. Let $(S, X)$ be a finite pointed $\omega$-semigroup associated by the main algorithm with the signed ordinal $\left[\varepsilon_{X}\right] \xi_{X}$. Any normal form of $(S, X)$ is bisimilar to the tree representation of $\left[\varepsilon_{X}\right] \xi_{X}$.

Proof. We use the notation of Algorithm 4.1 again. Let $G$ be a normal form of $(S, X)$, and $G^{\prime}$ be the tree representation of $\left[\varepsilon_{X}\right] \xi_{X}$. After computation of the main algorithm, the roots $r$ and $r^{\prime}$ of $G$ and $G^{\prime}$ are both associated with the signed ordinal $\left[\varepsilon_{X}\right] \xi_{X}$. Moreover, Lemma 5.2 shows that both graphs $G$ and $G^{\prime}$ satisfy the following properties: First, for every cut $[\varepsilon] \xi$ of $\left[\varepsilon_{X}\right] \xi_{X}$, there exists a node $n$ such that $\left[\varepsilon_{n}\right] \xi_{n}=[\varepsilon] \xi$. Second, any two nodes $n$ and $n^{\prime}$ satisfy $n \geq_{\mathcal{R}} n^{\prime}$ if and only $\left[\varepsilon_{n}\right] \xi_{n} \geq\left[\varepsilon_{n^{\prime}}\right] \xi_{n^{\prime}}$. In addition, by minimality of $G$ and by definition of $G^{\prime}$, every path in $G$ or in $G^{\prime}$ never visits a node associated with a non-cut of $\left[\varepsilon_{X}\right] \xi_{X}$; also, every path in $G$ or in $G^{\prime}$ never visits two nodes associated with the same cut of $\left[\varepsilon_{X}\right] \xi_{X}$. All these properties ensure the existence of the following winning strategy for Player II in $\mathbb{B I S}\left(G, G^{\prime}\right)$ : every time I moves to a successor node $n$, II moves to a successor node $n^{\prime}$ such that $\left[\varepsilon_{n}\right] \xi_{n}=\left[\varepsilon_{n^{\prime}}\right] \xi_{n^{\prime}}$. Therefore, $G \approx G^{\prime}$.

Theorem 7.6. Let $(S, X)$ be a finite pointed $\omega$-semigroup, and $N_{X}$ be a normal form of $(S, X)$.

(1) $d_{s g}(X)=\xi$ and $X$ is non-self-dual if and only if $N_{X}$ is bisimilar to the tree representation of $[+] \xi$ or $[-] \xi$.

(2) $d_{s g}(X)=\xi$ and $X$ is self-dual if and only if $N_{X}$ is bisimilar to the tree representation of $[ \pm] \xi$.

Proof. If $d_{s g}(X)=\xi$ and $X$ is non-self-dual, then $\left[\varepsilon_{X}\right] \xi_{X}$ is equal to $[+] \xi$ or $[-] \xi$. Hence, by Proposition 7.5, $N_{X}$ is bisimilar to the tree representation of $[+] \xi$ or $[-] \xi$. Conversely, assume that $N_{X}$ is bisimilar to the tree representation of $[\varepsilon] \xi$, with $\varepsilon \in\{+,-\}$. Proposition 7.5 shows that $N_{X}$ is also bisimilar to the tree representation of $\left[\varepsilon_{X}\right] \xi_{X}$. Hence, the tree representations of $\left[\varepsilon_{X}\right] \xi_{X}$ and $[\varepsilon] \xi$ are bisimilar, and Lemma 7.4 proves that $\left[\varepsilon_{X}\right] \xi_{X}=[\varepsilon] \xi$, where $\varepsilon \in\{+,-\}$. Therefore, $d_{s g}(X)=\xi$, and $X$ is non-self-dual. The second case is proved analogously.

Theorem 7.7. Let $(S, X)$ and $(T, Y)$ be two finite pointed $\omega$-semigroups with normal forms $N_{X}$ and $N_{Y}$, respectively. Then $X \equiv_{S G} Y$ if and only if $N_{X} \approx N_{Y}$.

Proof. If $X \equiv_{S G} Y$, then $\left[\varepsilon_{X}\right] \xi_{X}=\left[\varepsilon_{Y}\right] \xi_{Y}$. Hence, the tree representations $T_{X}$ and $T_{Y}$ of $\left[\varepsilon_{X}\right] \xi_{X}$ and $\left[\varepsilon_{Y}\right] \xi_{Y}$ are equal. Proposition 7.5 then implies $N_{X} \approx T_{X}=T_{Y} \approx$ $N_{Y}$. Conversely, by Proposition 7.5 again, one has $T_{X} \approx N_{X} \approx N_{Y} \approx T_{Y}$. Thus $T_{X} \approx T_{Y}$, and Lemma 7.4 shows that $\left[\varepsilon_{X}\right] \xi_{X}=\left[\varepsilon_{Y}\right] \xi_{Y}$. Therefore, $X \equiv_{S G} Y$. 

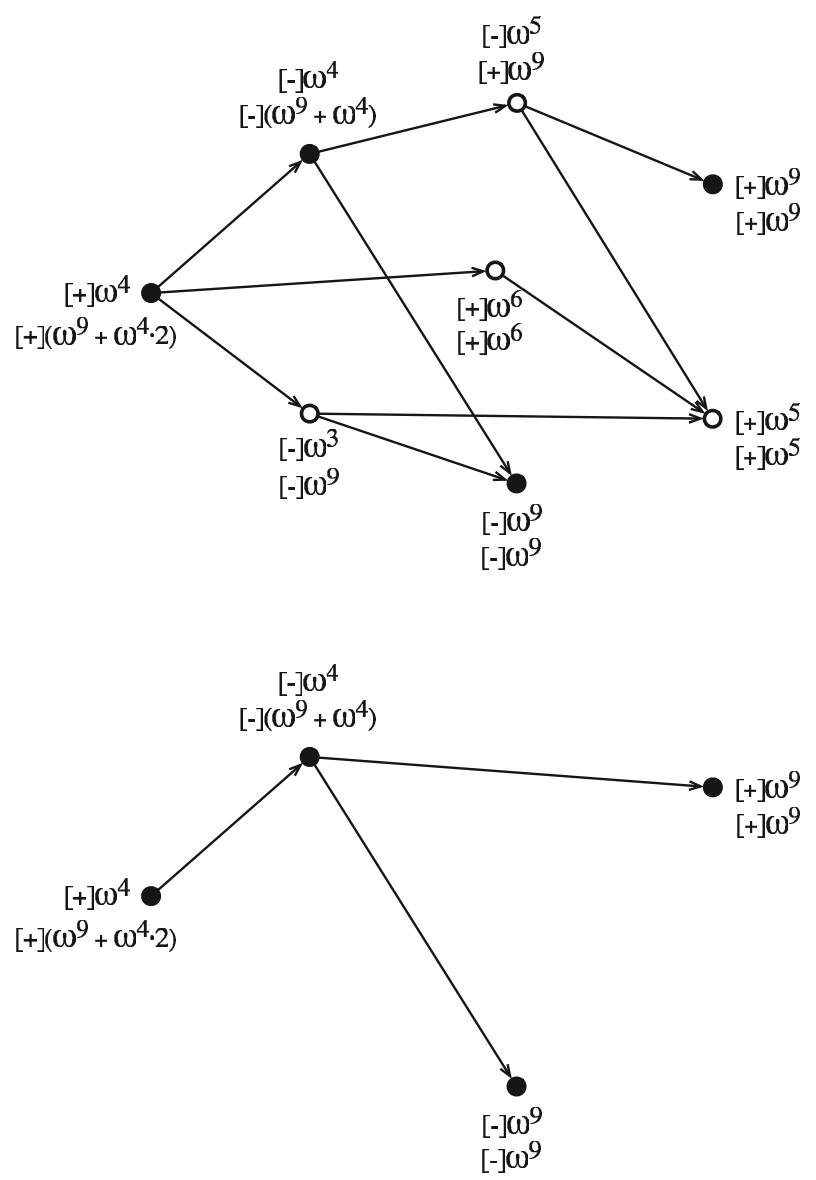

Figure 25. The signed DAG representation of a finite pointed $\omega$-semigroup $(S, X)$, and its normal form.

Corollary 7.8. Let $K$ and $L$ be two $\omega$-rational languages, let $\operatorname{synt}(K)$ and $\operatorname{synt}(L)$ be their syntactic images, and let $N_{K}$ and $N_{L}$ be the normal forms of $\operatorname{synt}(K)$ and $\operatorname{synt}(L)$. Then $K \equiv_{W} L$ if and only if $N_{X} \approx N_{Y}$.

Proof. One has $K \equiv_{W} L$ if and only if $\operatorname{synt}(K) \equiv_{S G} \operatorname{synt}(L)$. Theorem 7.7 leads to the conclusion.

Example 7.9. Figure 25 (top) illustrates the signed DAG representation of a finite pointed $\omega$-semigroup $(S, X)$. The two signed ordinals associated with each node are the outcomes of procedures (2) (top) and (3) (bottom) of the main algorithm. One has $\left[\varepsilon_{X}\right] \xi_{X}=[+]\left(\omega^{9}+\omega^{4} \cdot 2\right)$. Figure 25 (bottom) illustrates the normal form of $(S, X)$, which is bisimilar to the tree representation of $[+]\left(\omega^{9}+\omega^{4} \cdot 2\right)$. One has $d_{s g}(X)=\omega^{9}+\omega^{4} \cdot 2$, and $X$ is non-self-dual. 

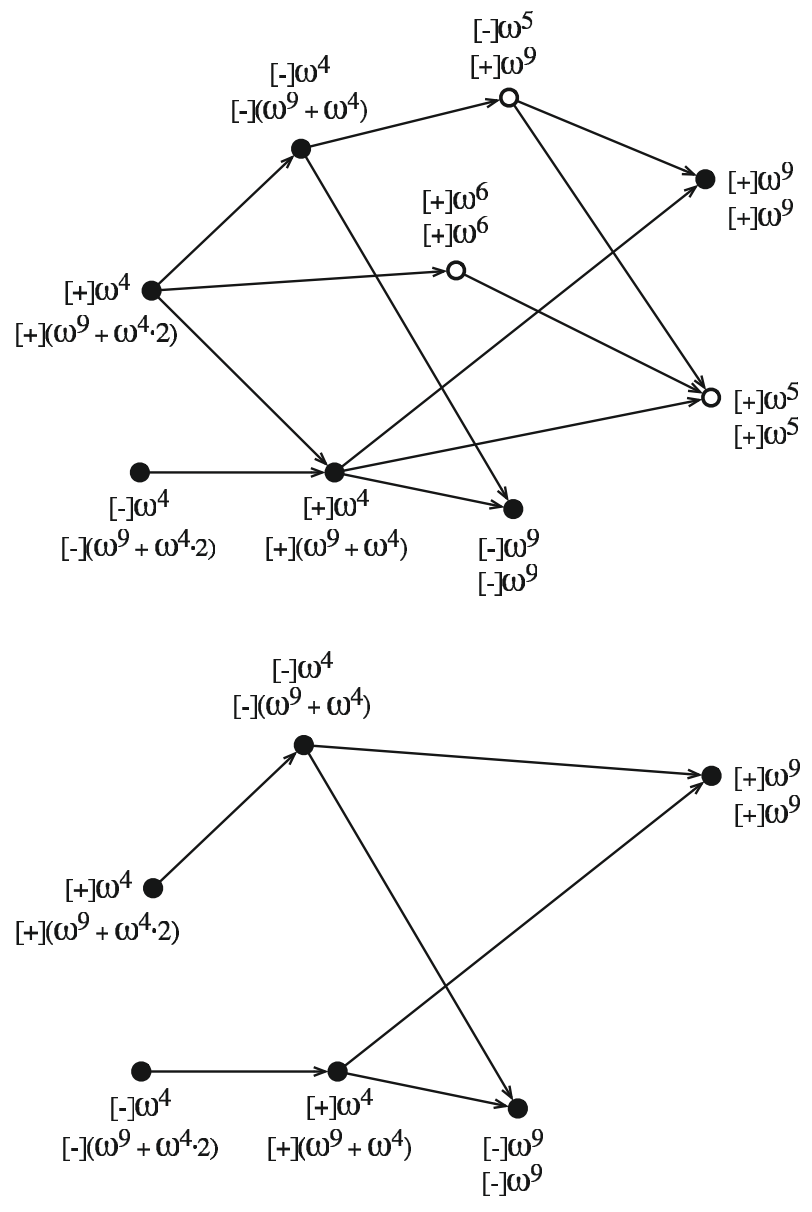

Figure 26. The signed DAG representation of a finite pointed $\omega$-semigroup $(T, Y)$, and its normal form.

Example 7.10. Again, Figure 26 (top) illustrates the signed DAG representation of a finite pointed $\omega$-semigroup $(T, Y)$. One has $\left[\varepsilon_{Y}\right] \xi_{Y}=[ \pm]\left(\omega^{9}+\omega^{4} \cdot 2\right)$. Figure 26 (bottom) illustrates the normal form of $(T, Y)$, which is bisimilar to the tree representation of $[ \pm]\left(\omega^{9}+\omega^{4} \cdot 2\right)$. In this case, one has $d_{s g}(Y)=\omega^{9}+\omega^{4} \cdot 2$, and $X$ is self-dual.

Example 7.11. Consider the finite pointed $\omega$-semigroup

$$
(S, X)=\left(\left(\{0,1\},\left\{0^{\omega}, 1^{\omega}\right\}\right),\left\{0^{\omega}\right\}\right)
$$

given in Example 3.3. The signed DAG representation and the normal form of $(S, X)$ are illustrated in Figure 27. The normal form of $(S, X)$ and the tree representation of $[-] \omega$ are bisimilar. Therefore, $d_{s g}(X)=\omega$, and $X$ is non-self-dual. 


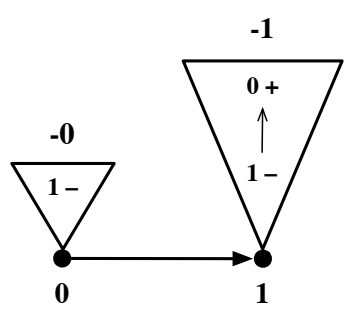

Figure 27. The signed DAG representation of $(S, X)$ and its normal form of reduced to the single node 1 labeled by -1 .

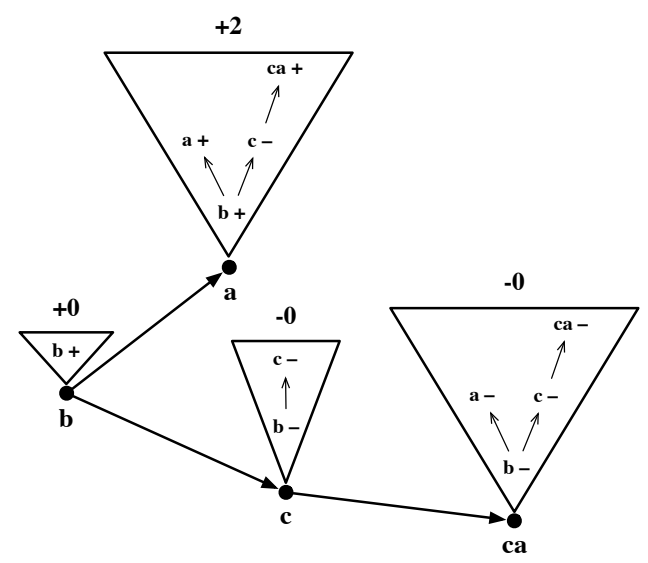

$\stackrel{+2}{\bullet}$

Figure 28. The signed DAG representation of $(T, Y)$ and its normal form reduced to a single node $a$ labeled by +2 .

Example 7.12. Consider the finite pointed $\omega$-semigroup

$$
(T, Y)=\left(\left(\{a, b, c, c a\},\left\{a^{\omega},(c a)^{\omega}, 0\right\}\right),\left\{a^{\omega}\right\}\right)
$$

given in Example 3.4. The signed DAG representation and the normal form of $(T, Y)$ is illustrated in Figure 28. The normal form of $(T, Y)$ is bisimilar to the tree representation of $[+] \omega^{2}$. Therefore, $d_{s g}(X)=\omega^{2}$, and $X$ is non-self-dual. 


\section{Conclusion}

We hope this work provides a convincing description of the algebraic counterpart of the Wagner hierarchy. In the first paper, we initially proved that the Wagner degree of an $\omega$-rational language is indeed a syntactic invariant. We then defined a Wadge-like reduction on finite pointed $\omega$-semigroups and showed that the resulting algebraic hierarchy is precisely isomorphic to the Wagner hierarchy. This algebraic representative of the Wagner hierarchy is thence a well-founded and decidable partial ordering of height $\omega^{\omega}$. In particular, an $\omega$-rational language and its syntactic image are proven to share the same Wagner degree, and syntactic pointed $\omega$-semigroups appeared as minimal representatives of their Wagner classes, whereas there is no convincing notion of minimal Muller automata of a given Wagner degree. In the second paper, we described a graphical decision procedure of this hierarchy based on a graph representation of finite pointed $\omega$-semigroups. This algorithm may thus compute the Wagner degree of any $\omega$-rational language directly on its syntactic image. It consists of a reformulation in this algebraic context of Wagner's naming procedure [21]. Afterwards, we showed how to build finite pointed $\omega$-semigroups of any given degree. We finally described the algebraic invariant characterizing every degree of this algebraic hierarchy. These invariants are also a reformulation in this context of the notions of maximal $\xi$-chains presented in [8], or maximal $\mu_{\alpha}$-alternating trees described in [18], or also maximal binary tree-like sequences of superchains described in [21].

We notice that our graph representation of finite pointed $\omega$-semigroups seems more complex than the graph of Muller automata: the set of loops of a given strictly connected component in a Muller automata is a semi-lattice for inclusion, whereas the set of idempotents of a given $\mathcal{R}$-class of prefixes is not, since it contains several petals. The question of the existence of a DAG decomposition of finite $\omega$ semigroups looking exactly as complex as the graphs of Muller automata is still open.

This work can be extended in several directions. On the one hand, we hope to widen this analysis to more sophisticated $\omega$-languages, like the ones recognized by deterministic counters, or even deterministic pushdown automata (PDA). This would require a description of the corresponding infinite $\omega$-semigroups, since the Wadge hierarchies of deterministic $\omega$-languages accepted by counter automata or PDA are strictly finer than the Wagner hierarchy [6,9]. However, an extension of this work to languages recognized by nondeterministic PDA would be very challenging, since the Wadge hierarchy of $\omega$-context-free languages (those recognized by nondeterministic PDA) was proven to be as complicated as the Wadge hierarchy of $\omega$-languages accepted by nondeterministic Turing machines [10].

On the other hand, since the Wadge hierarchy coincides with the restriction of the $\mathbb{S} \mathbb{G}$-hierarchy to free $\omega$-semigroups, this work could also enlighten the Borel Wadge hierarchy itself, by characterizing Borel sets by precise algebraic properties. For instance, we already know that a Borel $\omega$-language $A$ is non-self-dual if and only if it is $\mathbb{S} \mathbb{G}$-equivalent to some set $B$ extracted from some $\omega$-monoid. Also, a Borel set $A$ has a Wadge degree of the form $\omega_{1}{ }^{\alpha}$, with $\operatorname{cof}(\alpha) \neq \omega$, if 
and only if it is $\mathbb{S} G$-equivalent to some set $B$ extracted from some $\omega$-group (this result involves more sophisticated considerations about initializability, as shown in $[5,7])$. Extending such results would require to provide, for any given Borel $\omega$-language $A$, an $\mathbb{S} G$-equivalent set $B$ extracted from a particular $\omega$-semigroup which algebraically characterizes the Wadge class generated by $A$.

Acknowledgements. The authors wish to express their profound gratitude to Jean-Eric Pin for his helpful participation in this whole work, and also to Victor Selivanov for helpful and interesting discussions.

\section{REFERENCES}

[1] J. Cabessa and J. Duparc, An infinite game over $\omega$-semigroups, in Foundations of the Formal Sciences V, Infinite Games, edited by S. Bold, B. Löwe, T. Räsch, J. van Benthem. Studies in Logic 11. College Publications, London (2007) 63-78.

[2] O. Carton and D. Perrin, Chains and superchains in $\omega$-semigroups, edited by Almeida Jorge et al., Semigroups, automata and languages. Papers from the conference, Porto, Portugal (1994) June 20-24. World Scientific, Singapore (1996) 17-28.

[3] O. Carton and D. Perrin, Chains and superchains for $\omega$-rational sets, automata and semigroups. Int. J. Algebra Comput. 7 (1997) 673-695.

[4] O. Carton and D. Perrin, The Wagner hierarchy. Int. J. Algebra Comput. 9 (1999) 597-620.

[5] J. Duparc, Wadge hierarchy and Veblen hierarchy. Part I: Borel sets of finite rank. J. Symbolic Logic 66 (2001) 56-86.

[6] J. Duparc, A hierarchy of deterministic context-free $\omega$-languages. Theoret. Comput. Sci. 290 (2003) 1253-1300.

[7] J. Duparc, Wadge hierarchy and Veblen hierarchy. Part II: Borel sets of infinite rank (to appear).

[8] J. Duparc and M. Riss, The missing link for $\omega$-rational sets, automata, and semigroups. Int. J. Algebra Comput. 16 (2006) 161-185.

[9] O. Finkel, An effective extension of the Wagner hierarchy to blind counter automata. In Computer Science Logic (Paris, 2001); Lect. Notes Comput. Sci. 2142 (2001) 369-383.

[10] O. Finkel, Borel ranks and Wadge degrees of context free omega languages. In New Computational Paradigms, First Conference on Computability in Europe, CiE. Lect. Notes Comput. Sci. 2142 (2005) 129-138.

[11] A.S. Kechris, Classical descriptive set theory, Graduate Texts in Mathematics 156. SpringerVerlag, New York (1995).

[12] K. Kunen, Set theory. An introduction to independence proofs. 2nd print. Studies in Logic and the Foundations of Mathematics 102. North-Holland (1983) 313.

[13] R.E. Ladner, Application of model theoretic games to discrete linear orders and finite automata. Inform. Control 33 (1977) 281-303.

[14] Y.N. Moschovakis, Descriptive set theory. Studies in Logic and the Foundations of Mathematics 100. North-Holland Publishing Company (1980) 637.

[15] D. Perrin and J.-E. Pin, First-order logic and star-free sets. J. Comput. System Sci. 32 (1986) 393-406.

[16] D. Perrin and J.-Éric Pin, Infinite words. Pure Appl. Mathematics 141. Elsevier (2004).

[17] J.-E. Pin, Varieties of formal languages. North Oxford, London and Plenum, New-York (1986).

[18] V. Selivanov, Fine hierarchy of regular $\omega$-languages. Theoret. Comput. Sci. 191 (1998) 3759.

[19] W. Thomas, Star-free regular sets of $\omega$-sequences. Inform. Control 42 (1979) 148-156. 
[20] W.W. Wadge, Reducibility and determinateness on the Baire space. Ph.D. thesis, University of California, Berkeley (1983).

[21] K. Wagner, On $\omega$-regular sets. Inform. Control 43 (1979) 123-177.

[22] T. Wilke, An Eilenberg theorem for $\infty$-languages. In Automata, languages and programming (Madrid, 1991). Lect. Notes Comput. Sci. 510 (1991) 588-599.

[23] T. Wilke and H. Yoo, Computing the Wadge degree, the Lifshitz degree, and the Rabin index of a regular language of infinite words in polynomial time. In TAPSOFT '95: Theory and Practive of Software Development, edited by Peter D. Mosses, M. Nielsen, M.I. Schwartzbach. Lect. Notes Comput. Sci. 915 (1995) 288-302.

Communicated by Ch. Choffrut.

Received April 10, 2008. Accepted December 18, 2008. 